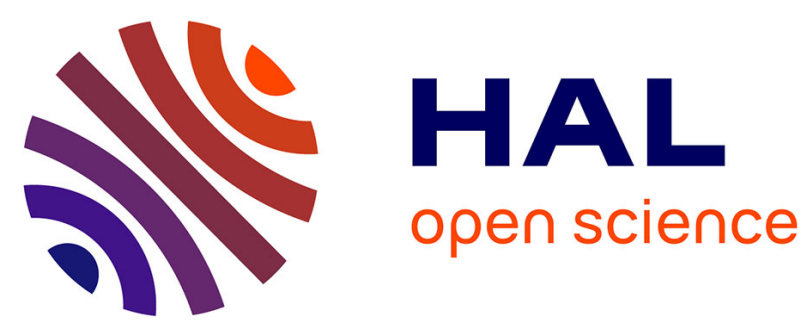

\title{
Temporal control of Dickeya dadantii main virulence gene expression by growth phase-dependent alteration of regulatory nucleoprotein complexes
}

\author{
A. Duprey, G. Muskhelishvili, S. Reverchon, W. Nasser
}

\section{To cite this version:}

A. Duprey, G. Muskhelishvili, S. Reverchon, W. Nasser. Temporal control of Dickeya dadantii main virulence gene expression by growth phase-dependent alteration of regulatory nucleoprotein complexes. Biochimica et Biophysica Acta - Gene Regulatory Mechanisms , 2016, 1859 (11), pp.1470-1480. hal01997030

\author{
HAL Id: hal-01997030 \\ https://hal.science/hal-01997030
}

Submitted on 15 Nov 2021

HAL is a multi-disciplinary open access archive for the deposit and dissemination of scientific research documents, whether they are published or not. The documents may come from teaching and research institutions in France or abroad, or from public or private research centers.
L'archive ouverte pluridisciplinaire $\mathbf{H A L}$, est destinée au dépôt et à la diffusion de documents scientifiques de niveau recherche, publiés ou non, émanant des établissements d'enseignement et de recherche français ou étrangers, des laboratoires publics ou privés. 
1 Temporal Control of Dickeya dadantii Main Virulence Gene Expression by Growth

2 Phase-Dependent Alteration of Regulatory Nucleoprotein Complexes

4 Short title: Control of Virulence Gene by Nucleoprotein Complex Alteration

Alexandre Duprey ${ }^{1,2,3}$, Georgi Muskhelishvili ${ }^{1,2,3}$, Sylvie Reverchon ${ }^{1,2,3}$ and William Nasser ${ }^{1,2,3 *}$

${ }^{1}$ Université Lyon 1, F-69622 Villeurbanne, France

2 INSA de Lyon, F-69621 Villeurbanne, France

${ }^{3}$ CNRS UMR5240 Microbiologie, Adaptation et Pathogénie, Villeurbanne, France

* To whom correspondence should be addressed. Tel: +33 (0)4 724326 95; Fax: +33 (0)4 724326 86; Email: william.nasser@insa-lyon.fr

\section{Abstract}

In bacteria, transcriptional control of important genes often involves numerous factors, forming an intricate web of interactions. Due to this complexity, the effects of transcriptional regulators are most often studied separately and little information is available concerning their interactions. In this work using a combination of biochemical and genetics approaches, we explore the regulation of $D$. dadantii major virulence gene pelD. By dissecting the individual effects of binding sites for the abundant nucleoid-associated protein FIS and the global transcriptional regulator CRP, as well as the impact of a newly discovered divergent promoter, div, we provide an unprecedented level of detail on the 
multifactorial regulation of gene transcription. We show that the temporal regulation of pelD is under the control of changing composition of higher-order nucleoprotein complexes formed on binding of FIS and CRP to the div and peID promoters during the growth cycle, that allows for sequential expression of div and pelD in the early and late exponential growth phases, respectively. Our results reveal the importance of "orphan" promoters in gene regulation and show that the individual binding sites for a regulator can serve several purposes and have different effects on transcription, thus adding a new level of complexity to bacterial transcriptional regulation.

\section{Graphical abstract}
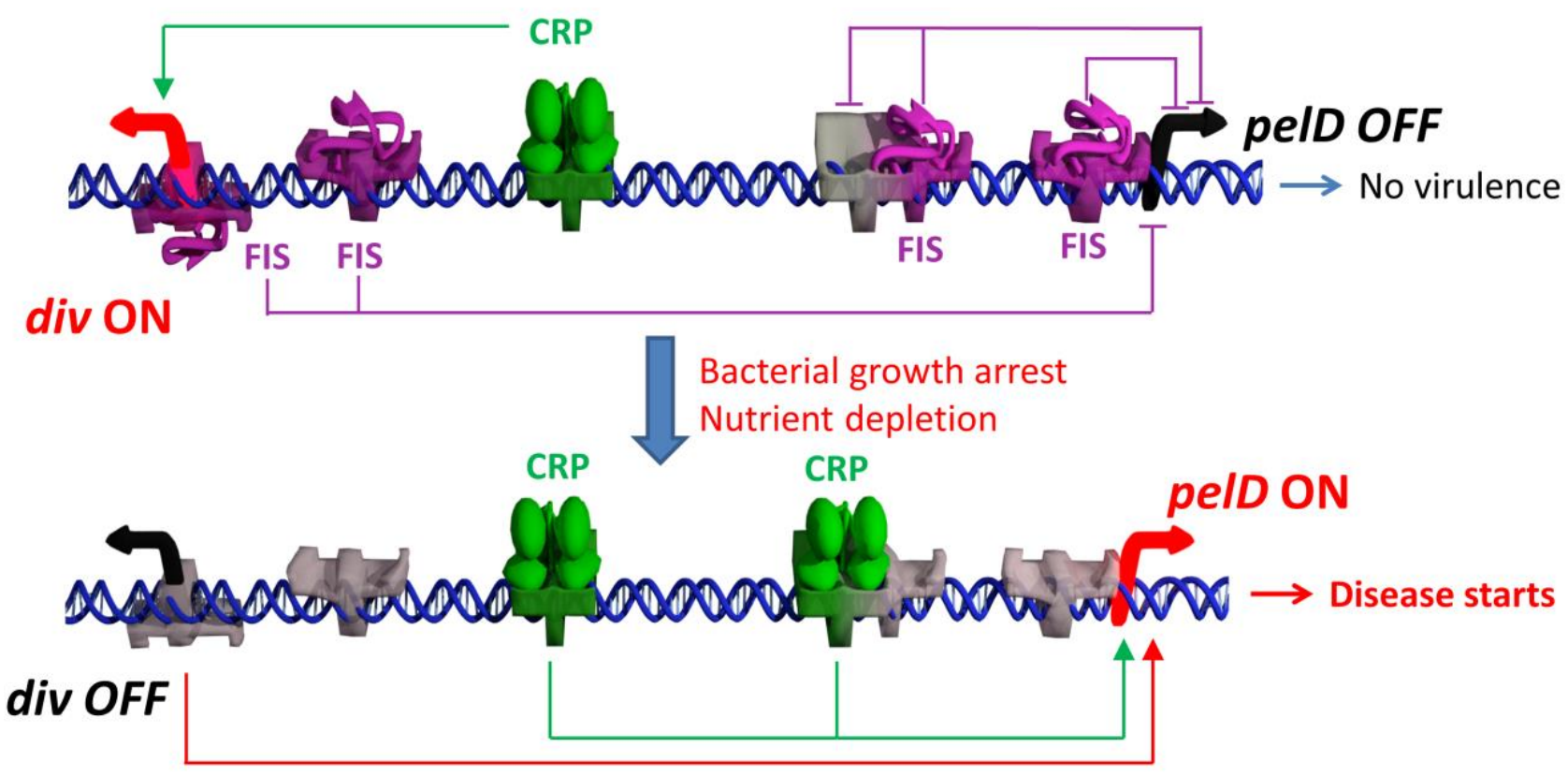

\section{Highlights}

- The transcriptional regulation of the peID virulence gene of $D$. dadantii is complex

- pelD is regulated by a divergent orphan promoter div 
- Individual FIS binding sites contribute differentially to peID regulation

- CRP activates pelD and div promoters asymmetrically

- Nucleoprotein complexes change to create a sequential expression pattern of pelD and div

\section{Keywords}

Transcriptional regulation, temporal regulation, Nucleoid-associated protein, FIS, CRP, virulence gene

\section{Abbreviations}

TF: Transcription Factor

NAP: Nucleoid-Associated Protein

PGA: PolyGalacturonic Acid

\section{Introduction}

During the bacterial life cycle, the cells can be confronted with a variety of environments differing in nutrient composition/availability, temperature, $\mathrm{pH}$, salinity and biotic interactions. Successful adaptation to these variable environmental conditions requires appropriate phenotypic modifications. For this purpose, bacteria modify the gene expression primarily at the level of transcriptional regulation, mediated by transcription factors (TFs) binding at regulatory site(s) in the gene promoter regions. Whereas repression by direct steric hindrance leading to promoter occlusion, and activation by recruitment of RNA polymerase by a TF, are the most frequently described regulation mechanisms [1], most genes are controlled by several TFs often competing for binding sites in the gene regulatory region, or for interaction with RNA polymerase. Furthermore, a 
small class of highly abundant TFs, known as nucleoid-associated proteins (NAPs), regulate transcription via a variety of DNA topology-related mechanisms such as looping [2], modulation of the spatial proximity of DNA-binding factors [3], and alteration of DNA configuration [4]. This potential of utilising topological changes in DNA entails complex regulatory mechanisms, which are not well understood.

FIS (factor for inversion stimulation) is one of the major NAPs in y-proteobacteria, capable of bending and looping the DNA and acting as a global regulator of transcription and growth [5-7]. In adition to direct binding effects, FIS can influence global DNA topology via regulation of DNA topoisomerases [8]. In particular, FIS acts to preserve the moderately supercoiled forms, protecting the DNA from shifts towards the strongly relaxed or highly negatively supercoiled extremes of the topological spectrum [9]. In E. coli, FIS is highly abundant in early to mid-exponential growth phase when the bacterial growth rate is high, whereas afterwards its level rapidly decreases becoming undetectable in stationary phase [10]. FIS can activate transcription initiation by various mechanisms such as recruitment of RNA polymerase at the promoter, induction of a conformational change in the promoter DNA, or both [11]. However, FIS is a dual regulator and can also act as a repressor counteracting the expression of inessential gene products during rapid growth [11]. Thus, the changing concentration of FIS during the growth cycle can both activate and repress the production of growth-related proteins and modulate the expression of virulence factors in a growth phase-dependent manner [5,12] leading to a sequential production of specific proteins during infection.

Dickeya dadantii is a phytopahogenic bacterium originating from tropical areas, which has been recently identified as a causal agent of crop damage in temperate regions (PQR - EPPO database on quarantine pests, available from 
http://www.eppo.int/DATABASES/pqr/pqr.htm). D. dadantii has a broad host spectrum, including economically important crops such as corn (Zea mays), potato (Solanum tuberosum) and chicory (Cichorium endivia) [13]. The main infection symptom is soft rot, which results from the action of extracellular enzymes, mainly pectate lyases (Pels) degrading pectin, an essential component of the plant cell wall. $D$. dadantii produces more than ten Pels with different biochemical properties and expression patterns $[14,15]$. The synthesis of $D$. dadantii Pels is fine-tuned according to environmental or metabolic signals [16], such as the presence of pectic compounds (via KdgR repressor) [17-19], and the oxidative and acidic stresses $[3,20]$. It was shown that the sugar catabolism regulator complex cAMP-CRP is the main direct activator of the pel genes [19,21]. Furthermore, the abundant NAP FIS modulates the expression of the pel genes in a growth phasedependent manner [22]. Both these factors bind to the pel gene regulatory regions making these genes ideal for exploring how bacteria integrate various mechanisms to control transcription initiation. Among the Pels, PelD production is most strongly affected by pectin derivatives and growth-phase, which makes it a key element in the induction of $D$. dadantii virulence $[23,24]$. The pelD gene is inactive during exponential growth when the bacterial density is low, and strongly expressed in early stationary phase when the bacterial density is sufficient to overcome the plant defence mechanisms. Previously, it was demonstrated that FIS binds two regions in pelD [25], but their impact in modulating pelD expression remains unclear. Furthemore, it was observed that RNA polymerase binds at the pelD upstream AT-rich region more tightly than at the pelD core promoter itself $[25,26]$. Notably, binding of CRP and FIS induces overlapping protection patterns in the region between the upstream RNA polymerase binding site and the pelD promoter. However, neither the role of these overlapping binding sites, nor the impact of upstream RNA polymerase binding 
site on pelD transcription have been elucidated.

In this work, by combining in vivo transcriptional activity assays using reporter genes with in vitro biochemical studies, we describe the complexity of the pelD promoter control showing that the key component in the temporal pattern of pelD expression is the dynamic balance between the binding of RNA polymerases at either pelD or a newly discovered upstream divergent promoter modulated by FIS, CRP and DNA topology. By integrating the individual effects of regulators resolved at a single binding site scale with the data on polymerase binding site occupation, we propose a model of pelD transcriptional regulation during the growth cycle.

\section{MATERIALS AND METHODS}

Bacterial strains, plasmids, media, culture conditions and molecular biology methods

Escherichia coli and Dickeya dadantii were cultured at $37^{\circ} \mathrm{C}$ in LB (Luria-Bertani, [27]) and $30{ }^{\circ} \mathrm{C}$ in M63S (M63 [27] supplemented with sucrose (S) $0.2 \% \mathrm{w} / \mathrm{v}$ ), respectively. Antibiotics were used at the following concentrations: Ampcillin $100 \mu \mathrm{g} / \mathrm{mL}$, Chloramphenicol $20 \mu \mathrm{g} / \mathrm{mL}$ or $4 \mu \mathrm{g} / \mathrm{mL}$ for $E$. coli and $D$. dadantii, respectively. Liquid cultures were grown in a shaking incubator (150 r.p.m.). Media were solidified by the addition of $1.5 \%$ agar $(\mathrm{w} / \mathrm{v})$.

Plasmids from the pKD series were obtained either by direct synthesis (pKD0, pKD1, pKD2, pKD3, pKD4 and pKD5) or by QuickChange mutagenesis. pKD7 was mutated from pKD0 using primers pelDfis1bismut FW and pelDfis1bismut REV. pKD9 and pKD10 were mutated from pKD5 and pKD7, respectively, using primers pelDFis1mutFW and pelDFis1mutREV. pKD24 was mutated from pKD0 using primers 35rev v2 FW and 35rev 
v2 REV (Table S1). pKD26 and pKD27 were mutated from pKD0 using primers 395/396 and 397/398, respectively. pKD28 was mutated from pKD26 using primers 397/398.

Plasmid pJV was constructed by amplifying the double terminator B0014 (http://parts.igem.org/Part:BBa_B0014) from the plasmid pIG50 [28] using primers B0014 FW and B0014 REV. The EcoRI-Pvull PCR product was then cloned into pJCD0 [29], yielding a plasmid with terminators on both sides suitable for in vitro transcription. Like all pUC-derived plasmids, pJV bears the noncoding RNA-I inside its replication origin which was used as an internal standard.

Plasmids from the pPD series, pID series, pJD series, pLD0 and pND0 were obtained by restriction assembly of the corresponding pKD insert into pProbe AT', pProbe AT' (with different restriction sites for the pPD or pID series, see Table S1, such that peID controls gfp transcription in the pPD series, and div controls gfp transcription in the pID series), pJV, pUCTer-Luc-Cm or pNB4, respectively, using standard molecular biology protocols [30].

\section{In vivo transcriptional activity measurements}

Overnight bacterial precultures in M63S were diluted to an $\mathrm{OD}_{600}$ of 0.03 in $\mathrm{M} 63 \mathrm{~S}+\mathrm{CaCl}_{2}$ $0.1 \mathrm{mM}+$ Polygalacturonic acid (PGA) $0.2 \% \mathrm{w} / \mathrm{v}$ (Dickeya dadantii) or LB (E. coli). The medium was supplemented with D-luciferin (Perkin-Elmer, USA) at a final concentration of $450 \mu \mathrm{g} / \mathrm{mL}$ when working with Luc. When required, novobiocin was added at the start of the culture, at a final concentration of $25 \mu \mathrm{g} / \mathrm{mL}$.

For fluorescence and luminescence, $200 \mu \mathrm{L}$ of this dilution were then distributed in a Cellstar 96-well plate (655090, Greiner Bio-one, Germany). The OD600, fluorescence and luminescence were monitored for $48 \mathrm{~h}$ using an Infinite 200 pro plate reader (Tecan, 
Switzerland). For fluorescence, the excitation and emission wavelengths were 485 and $530 \mathrm{~nm}$, with a bandwidth of 9 and $20 \mathrm{~nm}$, respectively.

The normalised fluorescence is defined as the ratio of the fluorescence of the considered promoter in early stationary phase (which corresponds to $20 \mathrm{~h}$ growth for a WT strain) by the $\mathrm{OD}_{600}$, normalised to the ratio calculated for the WT promoter in a WT strain (arbitrarily set as 100).

mRNA levels were computed from fluorescence data using the model described in [31]. Due to the stability of the Gfp used (> 20 days half-life, [32]), degradation was neglected. Because pelD and div are not expressed at the same time (see Fig 4a), the maximum expression of both promoters is usually represented in the figures, which means early log growth phase for div and late log for pelD.

For GUS, the specific beta-glucuronidase activity of the whole culture was measured at a comparable growth stage using the method described in [33].

\section{DNase I footprinting}

For quantitative fluorescent DNase I footprinting, the protocol published in [19] was modified as follows. Fluorescently labelled primers DNase pel FW and DNase pel REV (Table S1) were used to amplify the pelD regulatory region from plasmids of the pKD series by PCR. A clean-up was then performed using the Nucleospin Gel and PCR Cleanup kit (Macherey-Nagel, the Netherlands). $50 \mathrm{ng}$ of the labelled DNA was used per reaction, which represents a 2-fold increase in DNA quantity compared to radioactivity. Protein concentrations were adapted accordingly. In the end, the samples were analysed on an $A B 3730 \mathrm{XL}$ capillary sequencer (Applied biosystems, USA). Peak areas were 
normalised to the total fluorescence of each sample.

\section{Potassium permanganate footprinting}

Potassium permanganate reactions were performed on supercoiled plasmid, in the conditions described in [25]. The reaction products were divided into 2 equal parts. 15 cycles of primer extension were performed with Phusion polymerase (New England Biolabs, USA) with the fluorescently labeled primers DNase pel REV, for one part, and Bla3B4 fluo for the other part (which was used as a standard). After the primer extensions, both reactions were mixed together and analysed on an AB $3730 \mathrm{XL}$ capillary sequencer (Applied biosystems, USA).

For in vivo reactivity assays, D. dadantii wild type cells harbouring plasmid pKD0, containing the full pelD and div promoters, were grown in minimal M63 medium containing sucrose $(0.2 \%, W / V)$ and polygalacturonate $(0.2 \%, W / V)$ until the required stages. Then $\mathrm{KMnO} 4$ was added at $10 \mathrm{mM}$ (final concentration) for $1 \mathrm{~min}$ as described [34] to the batch cultures growing at $30^{\circ} \mathrm{C}$ under vigourous shaking. The cells were collected by centrifugation and the plasmid was extracted with Machery Nalgel Spin Miniprep Kit. The isolated plasmids (125 $\mathrm{ng}$ ) were used as template for 5 cycles of primer extension with Taq polymerase (New England Biolabs, USA) and ${ }^{32}$ P-labeled primer 300 (Table S1). Reaction products were then loaded onto a sequencing gel.

\section{In vitro transcription}

Supercoiled pJD plasmids were incubated with CRP (purified from D. dadantii, used at 100 $\mathrm{nM}$ concentration unless indicated otherwise) and RNA polymerase (commercial holoenzyme from E. coli purchased from Epicentre, used at $40 \mathrm{nM}$ concentration unless 
210 indicated otherwise) for $5 \mathrm{~min}$ at $30{ }^{\circ} \mathrm{C}$ in a reaction buffer containing $10 \mathrm{mM}$ Tris- $\mathrm{HCl} \mathrm{pH}$

211 8, $0.5 \mathrm{mM}$ dithiothreitol, $150 \mathrm{mM} \mathrm{KCl}, 200 \mu \mathrm{M}$ cAMP and 0.1\% (v/v) Nonidet P-40.

212 Transcription was initiated by the addition of $0.2 \mathrm{mM} \mathrm{ATP,} \mathrm{GTP} \mathrm{and} \mathrm{CTP,} 0.01 \mathrm{mM}$ cold

$213 \mathrm{UTP}$ and $0.1 \mu \mathrm{Ci} / \mu \mathrm{L}$ [a-P $\left.{ }^{32}\right]-U T P$ and allowed to proceed for $15 \mathrm{~min}$. Reactions were

214 stopped by the addition of an equal volume of $20 \mathrm{mM}$ EDTA, $0.025 \%(\mathrm{w} / \mathrm{v})$ bromophenol

215 blue and $0.025 \%(\mathrm{w} / \mathrm{v})$ Xylene cyanol in formamide and loaded on a sequencing gel.

\section{RESULTS}

\section{Identification and inactivation of the FIS binding sites}

The cooperative binding of CRP at two distinct sites in the pelD regulatory region was established in a previous study [25]. Furthermore, it was found that binding of FIS resulted in protection of two DNA regions (from position -150 to -119 and -48 to +7 relative to the transcription initiation site at +1 ), one of which overlapped the pelD promoter (Fig 1). However, the exact number of FIS binding sites and their functional role remained unknown.

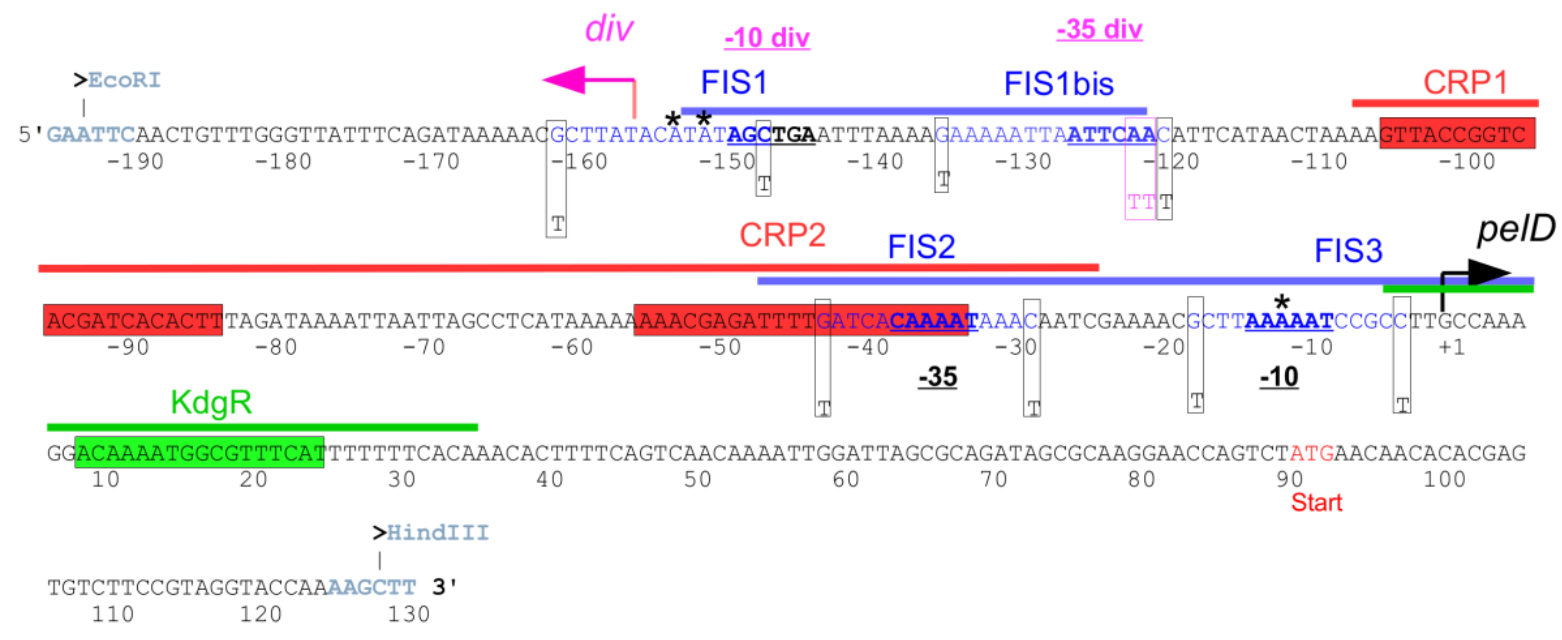

Fig 1: Structure of the pelD regulatory region. The sequence of the pelD regulatory region is annotated 
with the experimentally verified binding sites of CRP (red box) and KdgR (green box) (19, 26). The -10 and 35 elements are in bold and underlined, the translational start codon is in red letters, and the transcription initiation site is indicated by a broken arrow [26]. The positions of the $-35,-10$ elements and initiation site of the div promoter are indicated in pink. The two regions protected by FIS [25] and harbouring the four identified FIS binding sites are indicated by blue solid lines. Mutated positions are enclosed in a rectangle (black and pink for inactivation of FIS sites and div promoter, respectively). The region protected by CRP [25] and harbouring two CRP binding sites is indicated by red solid line. The gray boxes indicate A/T tracts centered at $+9,+18$ and +26 downstream of div initiation start site. Stars indicate the main Ts targeted by potassium permanganate in Fig 6, which are situated on the reverse strand. regulatory region was performed via Virtual Footprint (http://prodoric.tubs.de/vfp/vfp_promoter.php [35]), using the weight matrix established for E. coli. Only the sites with a score greater than 3.00 were considered (the mean score for referenced FIS binding sites being 2.87). This highlighted three binding sites, which were designated FIS1, FIS2 and FIS3 (Fig 1). FIS2 and FIS3 matched the previously determined FIS I experimental footprint [22], whereas FIS1 was located upstream partially overlapping the FIS II experimental footprint [25]. Manual examination of the sequence revealed a $4^{\text {th }}$ potential site, named FIS1bis, that was not detected by Virtual Footprint due to its increased length (16 bp) compared to the consensus FIS site (15 bp). The position of FIS1bis matches the previously identified experimental FISII footprint [25]. Among these four putative FIS binding sites, both FIS1 and FIS1bis are located far upstream, whereas FIS2 and FIS3 overlap the peID core promoter. Importantly, FIS2 also overlaps the CRP2 binding site. These four putative sites were inactivated separately, or in various combinations, by substituting the conserved $\mathrm{G}$ at the position 1 and the conserved $\mathrm{C}$ at the 
position 15 by Ts [36], as described in Fig 1 .

The success of inactivation was confirmed by DNase I footprinting demonstrating a disappearance of protection by FIS for all of these sites (Fig S1a), except for FIS1bis which showed barely detectable FIS binding on the WT template. Inactivation of FIS1, FIS2 or FIS3 did not noticeably modify the FIS footprints at the other sites (Fig S1a), which implies that binding of FIS at these sites is largely independent. In addition, major differences in affinity were observed, with FIS2 and FIS3 having a higher affinity than FIS1 and Fis 1 bis (Fig S1b).

\section{The pProbe-Gfp reporter system allows accurate quantification of promoter activity} in $D$. dadantii

Previously, most of the quantitative transcriptional activity measurements in $D$. dadantii have been performed by using beta-glucuronidase (GUS) genomic insertions [22], or by quantitative RT-PCR (q-RT-PCR) [3,37]. Both methods are relatively timeconsuming, due to the need to recombine the numerous transcriptional fusions into the chromosome or to extract RNA, respectively. Alternatively, heterologous GUS fusions on plasmids have been successfully used in E. coli [38]. We explored two reporter genes, Green fluorescent protein (Gfp) and Firefly luciferase (Luc), which would allow to perform easy, plasmid-based, real-time and automated measurements of gene expression in $D$. dadantii. Gfp is a highly stable protein (> 20 days half-life, [32]), while GUS is moderately stable (4h half-life, [39]) and Luc is an unstable protein (10 min half-life, [40]). The expression of a peID transcriptional fusion with each reporter (Fig S2) in D.dadantii wild type (WT) and its fis derivative backgrounds was measured. We found that the amplitude of the differences was negatively correlated with the stability of the proteins (ranging from 
a fold change of 1.4 for Gfp to 7.0 for Luc). However this increased sensitivity comes at the cost of decreased accuracy, especially in the fis mutant, with the coefficient of variation being $20 \%$ for Luc, but only $7 \%$ for Gfp. This results in weaker statistical tests for the Luc system e.g. a Welch test comparing WT to fis- conditions gives $p<10^{-9}$ for Gfp but only $p<10^{-3}$ for Luc ( $n=12$ per condition for each reporter system). Consequently, to obtain reproducible results, the Gfp system was used for further measurements, since despite lower sensitivity even small (but statistically significant) differences in fluorescence could be assumed to be biologically relevant.

\section{The peID FIS binding sites are partially redundant}

We next investigated the role of the different FIS binding sites in the modulation of pelD expression. To achieve this, plasmid-borne gfp transcriptional fusions were constructed with the mutated pelD promoters and transformed in $D$. dadantii wild type (WT) and fis mutant strains. The fluorescence of the cells was measured in early stationary phase, when the transcription of pel genes is maximal [41]. The results are shown in Fig 2. 


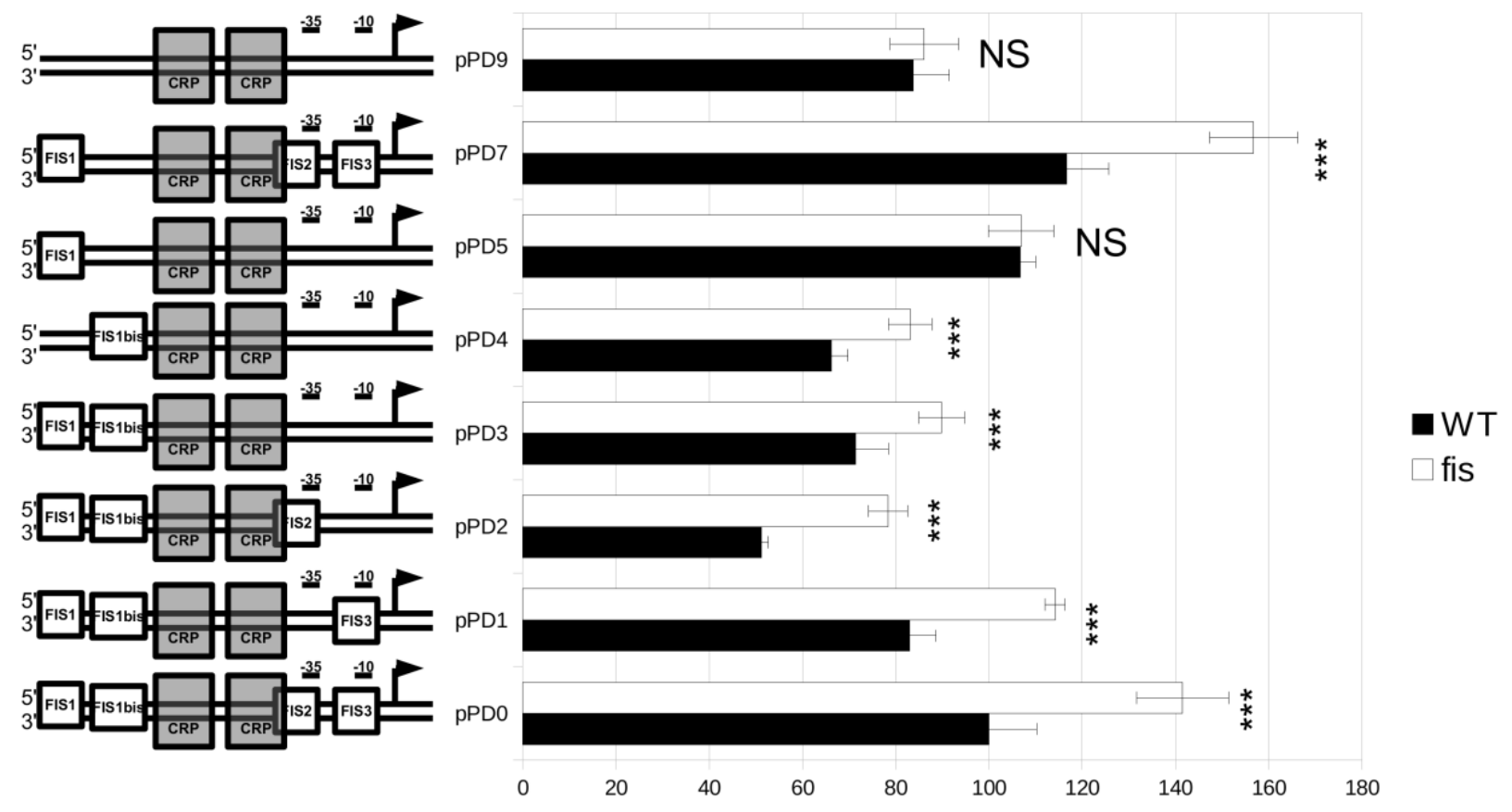

Normalised fluorescence/OD (AU)

Fig 2: Transcriptional activity of various pelD promoter constructs in $D$. dadantii wild type and fis mutant backgrounds. $D$. dadantii wild type and fis strains transformed with various pelD constructs (pProbe-Gfp plasmids D0 to D9 represented schematically on the left) were cultured in 96 well plates and the normalised fluorescence was determined in early stationary phase. Data represents the mean of 7 to 34 replicates and error bars represent the standard deviation. NS: not significant; ${ }^{* * *}$ : $p<0.001$ (Tukey's HSD test). Note that some of the introduced mutations affect the basal pelD promoter activity due to the overlap between the FIS binding sites and the core promoter sequence.

We found that as expected, FIS acted as a repressor [22], since the pelD activity was significantly increased in the fis mutant (see D0 in Fig 2). Inactivating either FIS1bis, FIS2 or FIS3 separately (compare D0 with D1, D2 and D7) had no significant impact on the repression by FIS (the fold-change remained constant at 1.40). Simultaneous inactivation of FIS2 and FIS3 (D3) alleviated the repression by FIS from 1.40-fold to 1.25fold ( $p<10^{-4}$, t-test for contrasts), and this residual repression can be attributed to the intact 
upstream sites. Furthermore, inactivation of FIS1, FIS2 and FIS3 had a similar effect as inactivation of FIS2 and FIS3 (compare D4 and D3), suggesting that FIS1 does not play any role in peID repression in vivo. However, only the simultaneous inactivation of FIS1bis, FIS2 and FIS3 resulted in a complete elimination of repression by FIS, comparable to the construct in which all FIS binding sites were inactivated (compare D5 and D9). It thus appears that FIS2, FIS3 and FIS1bis are required for full repression of the pelD promoter by FIS in vivo. In vitro transcription reactions performed with pelD plasmid constructs yielded similar results (Fig S3). Inactivation of both FIS2 and FIS3 together was necessary to fully remove the repression of peID by FIS, but, unlike in vivo, FIS1bis inactivation showed no effect, in keeping with the barely detectable occupancy of FIS1bis site in vitro (Fig S1).

\section{FIS competes with binding of CRP only at the CRP2 site}

Whereas the occupancy of either of the strong FIS2 and FIS3 sites overlapping the RNA polymerase (RNAP) recognition elements appears sufficient to prevent RNAP binding at the pelD promoter, FIS2 also overlaps the CRP2 binding site (see Fig 1), suggesting a potential competition between FIS and CRP for promoter binding. Therefore, quantitative DNase I footprinting was performed to examine whether the presence of FIS had an impact on the CRP footprint at the CRP2 site (Fig 3). On the wild-type regulatory region, a footprint by CRP at the CRP2 site was visible only in the absence of FIS, whereas on templates with inactivated FIS2 (or both FIS2 and FIS3) site(s), the protection of CRP2 site by CRP was observed despite the presence of FIS. In contrast, the protection of CRP1 site was independent of the presence of FIS on both the wild type and mutated templates (Fig S4). This suggests that binding of FIS at FIS2 specifically interferes with 

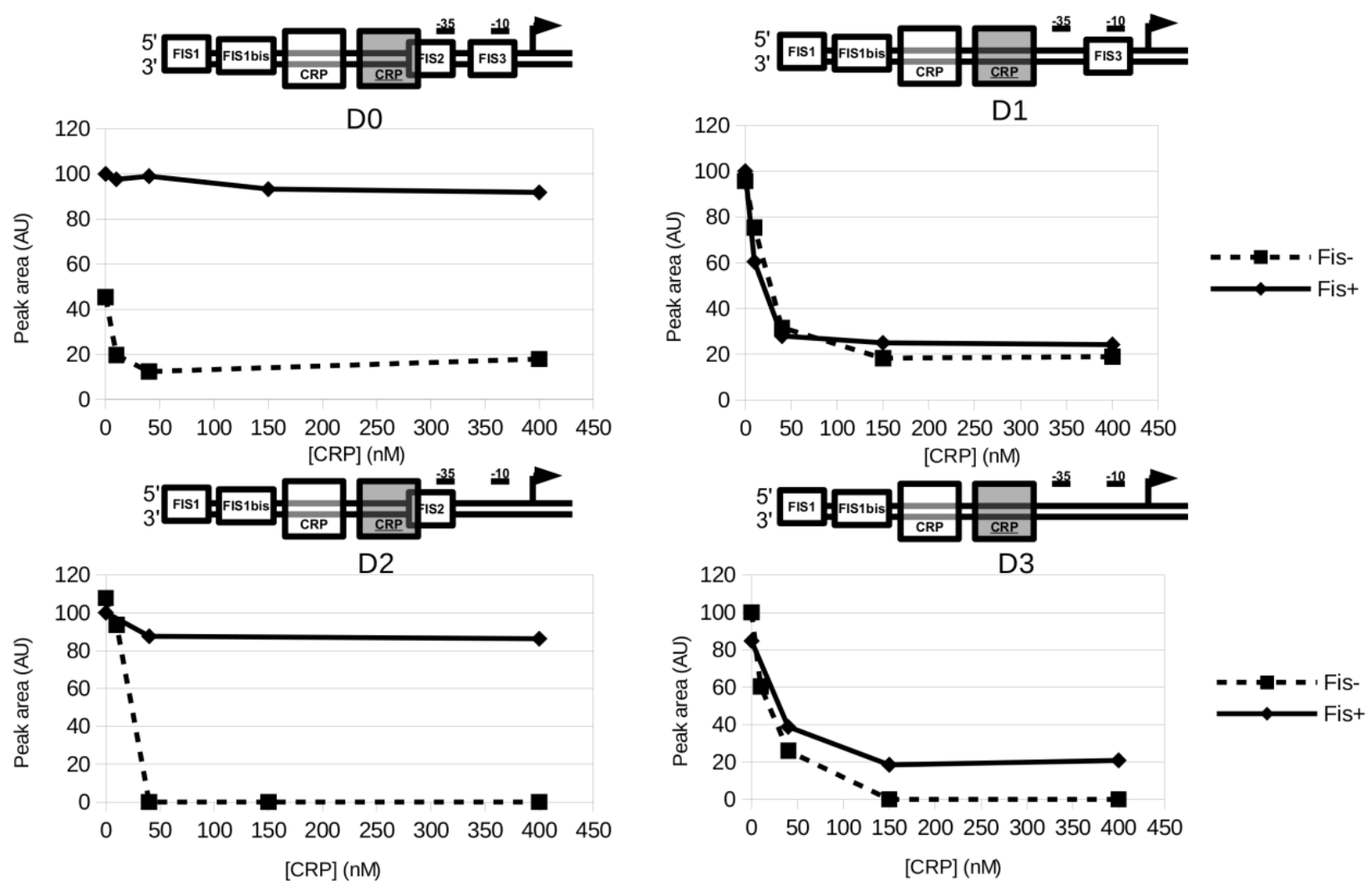

Fig 3: Modification of the CRP2 binding by FIS. Quantitative DNase I footprinting using fluorescently labelled linear peID DNA fragments lacking one or both of the FIS binding sites overlapping the peID promoter. The reactions were performed in the presence or absence of FIS (150 nM) and with increasing concentrations (from 0 to $400 \mathrm{nM}$ ) of CRP. The area of the peaks at one position within the CRP2 binding site (at position -29 relatively to the transcription start site) is represented for different CRP concentrations. This position is protected by CRP and becomes hypersensitive in presence of FIS. Absence of variation in this area (as observed with D0 and D2 in presence of FIS) means that CRP cannot bind the corresponding site. Consistent results were obtained also with two other positions analysed within the CRP2 footprint (-47 and -21). 
The upstream divergent promoter is activated by supercoiling and repressed by FIS

The upstream FIS binding sites (FIS1 and FIS1bis) are located too far upstream of pelD core promoter to be directly involved in competition with RNA polymerase for binding. However, previous DNAse I footprinting experiments have revealed that this upstream region contains a high affinity binding site for RNA polymerase (extending from pos. -142 to -104 ) in divergent orientation to pelD [26]. Indeed, recent RNAseq data analysis (Jiang et al. in preparation) confirmed by our in vitro transcription experiments identified a divergent transcript initiating from pelD upstream region and devoid of any meaningful ORF. We propose that this region comprises a divergent promoter (denoted hereafter as div), which potentially could influence the expression of pelD and also be regulated by FIS and CRP via FIS1/FIS1bis and CRP1 sites, respectively. Indeed FIS1/FIS1b overlap the 10 and -35 elements of the div promoter, whereas CRP1 is centred at -60.5 with respect to the div transcription initiation site (Fig 1). This latter organisational feature is characteristic of class I CRP-dependent promoters, suggesting that CRP1 site might be used for activation of both the pelD [26] and div promoters by CRP.

To examine the relationship between the pelD and div promoters we measured the amount of mRNA produced from both promoters on the plasmid constructs in vivo. We found that the peak of pelD expression observed as expected, on transition to stationary phase, was preceded by a peak of div expression, observed early during the exponential growth phase (Fig 4a), whereby the div promoter showed significantly lower expression levels than pelD. Measurements of the untwisting of the div and pelD promoters using in vivo $\mathrm{KMnO}_{4}$ reactivity assay (see Materials and methods) confirmed that the peak of div promoter untwisting during exponential phase preceded that of pelD occurring on transition 
to stationary phase (Fig S5). Interestingly, whereas these two promoters were sequentially activated during growth of $D$. dadantii, the decline of div activity clearly coincided with activation of pelD. We asked whether this correlation - given the close proximity of the promoters - indicated a possible link in their regulation mediated by binding proteins. Indeed, not only was the expression of both pelD and div increased in the fis mutant, but also the peak of div expression was shifted to the right along the growth curve (Fig 4a).

a)

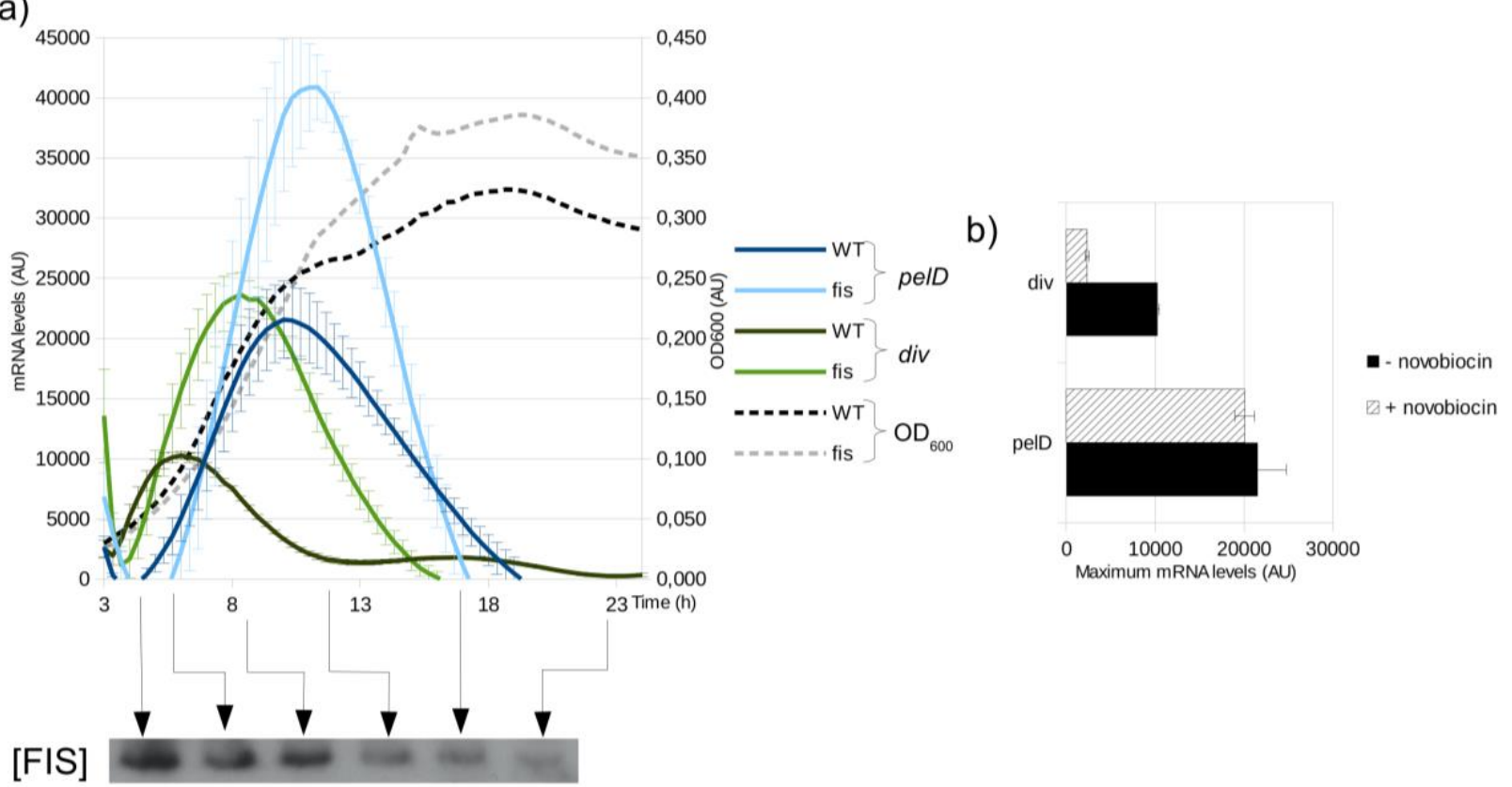

Fig 4: Transcriptional regulation of the div promoter. Dickeya dadantii wild type and fis mutant cells were transformed with constructs carrying pelD promoter-gfp or div promoter-gfp fusions and cultured in 96 well plates. Sublethal concentrations of novobiocin $(25 \mu \mathrm{g} / \mathrm{mL})$ were added at the start of the culture. a) The mRNA levels produced from different constructs in wild type and fis mutant cells were computed from fluorescence data as previously described [31]. Western blots indicating the levels of FIS at the indicated time points during the growth cycle. b) Maximum calculated mRNA levels obtained during the experiment in the presence or absence of novobiocin. 
It is noteworthy, that despite the de-repression of div promoter in the fis mutant, its activity sharply declined during the transition of cells to stationary phase, which is characterised by decrease in global DNA superhelicity (Fig 4a). Since unlike pelD, the peak of div activity was observed in early exponential growth phase characterised by high levels of negative DNA superhelicity, we compared the response of the div and pelD promoters to DNA relaxation (Fig 4b). We found that in contrast to pelD promoter, the div promoter activity was substantially decreased on DNA relaxation by novobiocin, a coumarin drug inhibiting the generation of negative superhelicity by DNA gyrase [42]. Therefore, whereas FIS clearly represses div promoter activity (Fig 4a), the observed reduction of div transcription later during the growth cycle (observed in both the wild type and fis mutant) could be explained by relaxation of DNA on transition of the cells to stationary phase [43].

\section{The two CRP binding sites synergistically activate both peID and div}

To investigate the impact of the CRP1 and CRP2 sites in pelD and div promoter expression we inactivated these sites either separately or together (see legend to Fig 5). The resulting constructs were used to quantify the promoter expression in the wild type strain. The data are shown in Fig 5. We found, as expected, that inactivation of either CRP1 or CRP2 separately strongly impairs the pelD promoter activity. However, this effect is more pronounced in the absence of CRP2. This observation is consistent with observed higher affinity of CRP2 compared to CRP1 (compare Fig 3 and Fig S4) and its optimal position (centred at $-42.5 \mathrm{bp}$ ) for activating the peID promoter. It thus appears that whereas CRP2 is the main activator site, both CRP binding sites synergistically activate the pelD promoter. At the same time, the CRP1 and CRP2 sites also act synergistically on the div 
409 promoter but in this case the impact of CRP1 site is predominant, in keeping with its 410 optimal location (-60.5bp) with regard to the div transcription initiation site (see Fig 1).

411 Thus, binding of CRP to CRP1 and CRP2 sites synergistically modulates both pelD and 412 div promoter expression, although the impact of individual sites at each promoter is 413 different.

a)

CRP1 GTTACCGGTCACGATCACACTT inactivated GTTACCGGTCACGAagtCACTT

CRP2 AAACGAGATTTTGATCACAAAA inactivated AAACGAGATTTTGAagtCAAAA consensus nnnTGTGAnnnnnnTCACAnnn b)

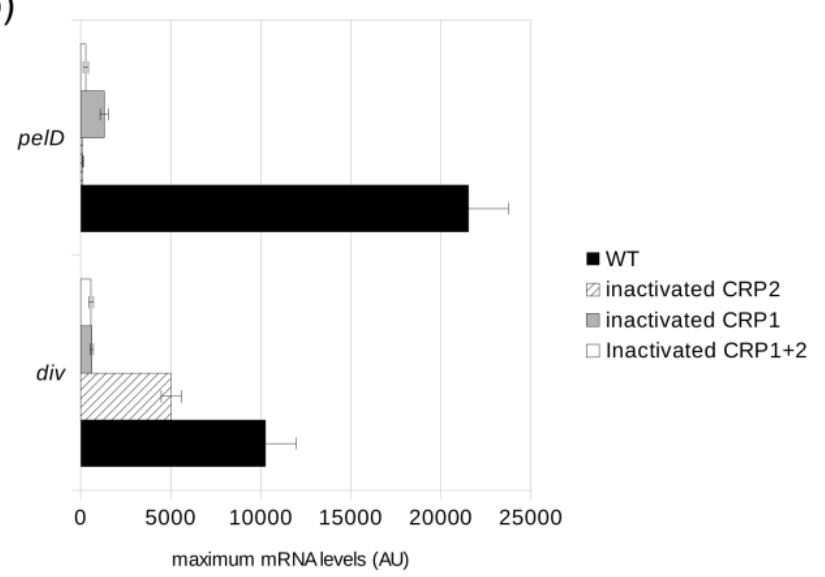

Fig 5: Individual contributions of the CRP1 and CRP2 sites on pelD and div expression. (a) CRP sites were inactivated by site-directed mutagenesis, substituting several of the most conserved nucleotides in the consensus, using the same approach as for FIS sites (see methods). (b) Expression of the mutated promoters was measured as in figure $6 \mathrm{~b}$. Data represents the mean of 10 replicates and error bars indicate standard deviation.

The divergent promoter potentially competes with peID for RNA polymerase binding in vitro

To gain more insight into the relationship between the div and pelD promoters we investigated in vitro the promoter opening using potassium permanganate reactivity assay. In these experiments, conducted with various concentrations of RNA polymerase and CRP, we observed that under conditions of low transcriptional activity (low concentrations 
427 of both CRP and RNAP), the opening of both promoters was similar, whereas under

a)

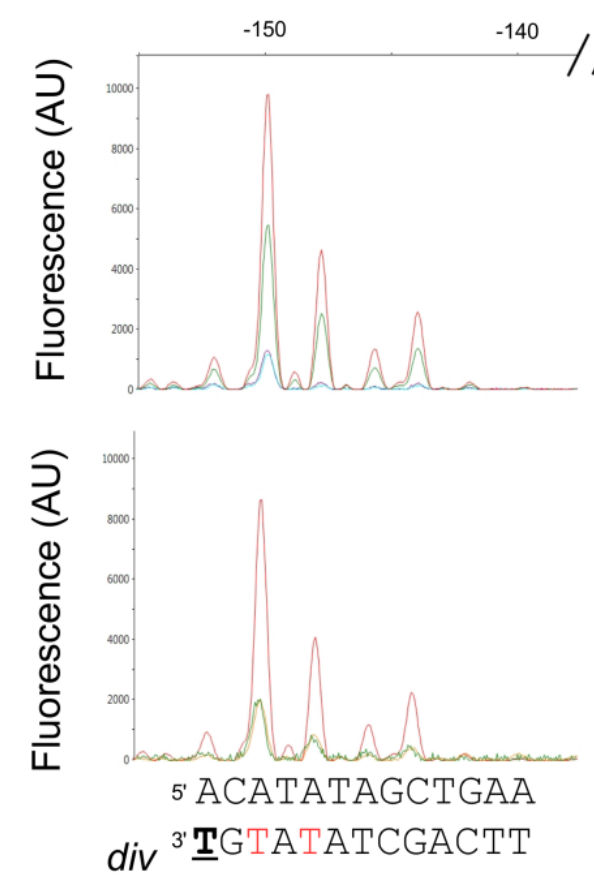
conditions favouring high levels of transcription (high concentrations of both RNAP and $\mathrm{CRP}$ ), an up to 6 -fold stronger reactivity to $\mathrm{KMnO}_{4}$ is observed at the div promoter compared to the pelD promoter (Fig 6). These results suggest that under conditions supporting active transcription in vitro, the div promoter can gain an advantage over the peID promoter in binding RNA polymerase.

Fig 6: Preferential binding of RNA polymerase at div does not correlate with stronger transcription. a)

Potassium permanganate reactivity of the supercoiled pelD regulatory region in the presence of varying concentrations of CRP and RNA polymerase as indicated on the right of the figure. The pelD and div transcription initiation sites are underlined in the corresponding sequences shown below the reactivity profiles. Both starnds are shown and attacked Ts are indicated in red. The capillary electrophoresis fluorescence profiles were normalised to the pelD peaks and overlaid with the position of each peak relatively to the pelD transcription start site. b) In vitro transcription using the supercoiled wild type peID promoter was performed in the presence of $40 \mathrm{nM}$ RNA polymerase and varying concentrations of CRP. The 
peID, RNA-I (internal standard) and div transcripts are shown.

In the in vitro transcription reactions performed in parallel, we found that although

\section{Inactivation of div reduces the peID activity in vitro}

To directly assess the effect of the div promoter on pelD activity we compared the promoter function of wild type peID construct and a construct with a mutation (D24) inactivating the -35 element of the div promoter and demonstrating a strong reduction of div opening by $\mathrm{KMnO}_{4}$ reactivity assay (Fig 7a). 

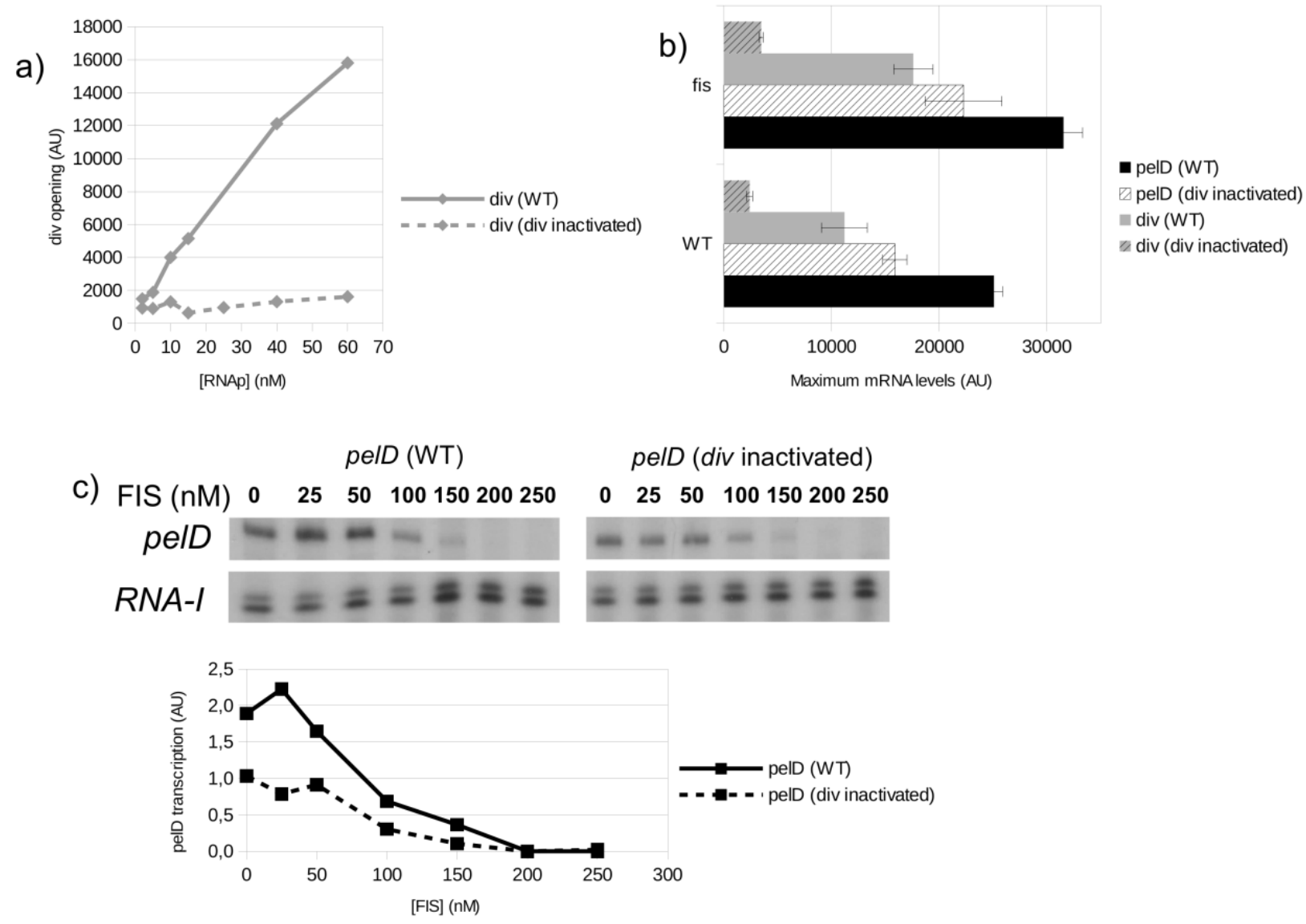

Fig 7: Impact of div on peID transcription. The div promoter was inactivated by mutating the -35 box (see Fig 1) and the impact of this mutation was assessed. a) Potassium permanganate reactivity (opening) of the wild type and inactivated div promoter in the presence of $100 \mathrm{nM}$ CRP and varying concentrations of RNA polymerase. The promoter opening was quantified as described in the legend to Fig 4. b) In vivo transcription of pelD and div constructs with intact and inactivated div promoter carried out under the same conditions as in Fig 4a. pelD and div expression was measured in real-time for 48 hours, like as in Fig 4a. Because pelD and div are not expressed at the same time (see Fig 4a), the maximum expression of both promoters is taken for this graph, which means early log for div and late log for peID. c) In vitro transcription of pelD and div constructs with intact and inactivated div promoter in the presence of $100 \mathrm{nM}$ CRP, $40 \mathrm{nM}$ RNA polymerase and varying concentrations of FIS.

Using both these constructs we compared the pelD mRNA levels produced in wild type and fis mutant cells. We observed that inactivation of div decreased pelD transcription 
475 in both the $D$. dadantii wild type and fis mutant cells (Fig 7b), indicating that div exerts an 476 activating effect on peID transcription and that activation is largely independent of FIS (this 477 effect was slightly more pronounced in wild type cells showing an 1.56-fold increase in pelD expression in presence of an intact div in WT cells, and an 1.41-fold in the fis cells).

Using both these constructs we also performed in vitro transcription experiments (Fig 7c). With the construct carrying inactivated div we observed a 2-fold decrease of pelD transcription compared to that with intact div, confirming in a purified system that div activates pelD.

\section{DISCUSSION}

The purpose of this work was to provide an integrated picture of the temporal regulation of the pelD promoter driving the expression of a major virulence factor of the plant pathogen $D$. dadantii. Upstream of the pelD promoter we identified a divergent promoter (div) which is not associated with any meaningful ORF and the activity of which is strongly reduced by DNA relaxation in vivo. This sensitivity to supercoiling is consistent with maximum div activity during early exponential phase, when the DNA superhelical density is maximal, and its sharp reduction during transition of cells to stationary phase and associated DNA relaxation [43]. In contrast, the pelD promoter is activated on transition to stationary phase concomitantly with the decline of div promoter activity. We found that the relationship between the closely spaced div and pelD promoters is not trivial, as under conditions supporting high rates of transcription in vitro, the div promoter binds RNAP and forms open complexes much more efficiently than pelD and could thus potentially compete with the latter (Fig 6a). However, inactivation of the div promoter 
significantly decreases pelD promoter activity (Fig $7 \mathrm{bc}$ ). Furthermore, despite the facile formation of open complexes by RNAP, the div promoter is considerably less active than pelD in vivo (Fig 4a) and is barely transcribed in vitro (Fig 6b). The reason for this discrepancy is not entirely clear, but the structural organisation of div strongly resembles that of a divergent promoter identified in the upstream region of the E. coli fis operon [45]. Both these weakly transcribed promoters demonstrate similar intrinsic properties such as overly strong untwisting in conjunction with reasonably close-to-consensus -35 hexamer sequences (TTGCAT for the fis divergent promoter, TTGAAT for the pelD divergent promoter).

In addition to studying the effects of two previously described CRP binding sites on peID and div, we identified four FIS binding sites, two of which (FIS2/3) overlap the pelD promoter sequence, and two are located upstream (FIS1/1 bis) and overlap with the div promoter. The FIS2 and FIS3 sites act as repressor sites inhibiting the peID expression during exponential growth phase (Fig 4a), whereas the FIS1 and FIS1bis sites, although weak, can repress div transcription during early exponential growth in vivo, when the FIS concentration is high (Fig 4a). However, it is noteworthy that inactivation of FIS1bis site affected pelD activity only in vivo (compare pPD4 and pPD5 in Fig 2) but not in vitro (compare pJD3 and pJD9 in Fig S3). This could be attributed to differences in the in vitro and in vivo conditions and indicate more complex relationships, perhaps involving changes of other regulators not known at this time.

Repression of peID by FIS appears to be mediated by two mechanisms. The FIS2 site is the main repressor site preventing the binding of both the RNA polymerase and the activator CRP at CRP2 site, whereas FIS3 site prevents the binding of RNA polymerase, but does not interfere with CRP binding. The second CRP binding site (CRP1) is suitably 
positioned to activate both the div and pelD promoters by providing interaction surfaces for the C-terminal domains of the $\alpha$ subunits ( $\alpha$-CTDs) of RNAP [46]. Indeed, our data suggest that binding of CRP at CRP1 and CRP2 synergistically activate both the pelD and div promoters. However, the relative contribution of these two sites to the activity of the pelD and div promoters is different. The pertinent question is whether the identified organisation of the DNA binding sites and the observed relationships between the binding molecules of RNAP and global regulators can be rationalised with regard to the critical function of pelD gene in bacterial virulence.

\section{Mechanism of growth phase dependent regulation of pelD}

For $D$. dadantii it is vital to completely repress the expression of pelD (as well as other pel genes) in exponential phase, especially since any leaky pel expression would trigger a massive production of Pels due to the KdgR positive feedback loop [17]. If the bacterial population density is low, an early activation of pel genes results in an early activation of the plant defense reactions and ultimately in bacterial death [47] as observed, for example, in D.dadantii mutant lacking the fis gene [22]. This justifies an establishment of redundant mechanisms for efficient pelD repression during exponential growth.

Our data suggest that $D$. dadantii uses three different mechanisms to prevent aberrant expression of pelD during exponential growth phase: i) direct hindrance of the RNA polymerase binding via FIS2 and FIS3, ii) competition between FIS and activator CRP for binding of the overlapping FIS2 and the CRP2 sites and, iii) potential competition of the div promoter with pelD promoter for RNA polymerase binding. Our observations allow us to propose a model for regulation of the expression of pelD by taking into account all these factors and the expression patterns of FIS (10) and the div promoter (Fig 8). CRP is obviously central for this regulation, as it is absolutely required for the activity of both the 
peID and div promoters. The DNA region with two CRP binding sites organised at optimal positions for activating two divergent ( $p e / D$ and div) promoters would serve as a trap for RNA polymerase directing transcription in one or another direction depending on the transcriptional environment. The expression peak of the div promoter in $D$. dadantii is observed at $6 \mathrm{~h}$ during early exponential phase, i.e. when FIS is near its maximum concentration (Fig 4a, lower panel). Given that FIS1/1bis are low affinity sites, high FIS concentrations perhaps weakly interfere with binding of RNAP to div but fully repress pelD via FIS2/3. In addition, in early exponential phase the DNA is highly supercoiled (9), allowing maximal div promoter activity mainly supported by CRP binding at CRP1 site centred at -60.5 with respect to the div transcription initiation site. Indeed, whereas FIS precludes the binding of CRP at CRP2 site and activation of pelD, it does not interfere with binding of CRP at CRP1 site and activation of div, consistent with different impact of individual CRP binding sites on the pelD and div promoter activity (Fig. 5b). At this growth stage the high-affinity div promoter can serve as an efficient trap withdrawing the RNA polymerase molecules that could otherwise bind at the pelD promoter. During midexponential phase the concentration of FIS is still sufficient to repress the peID promoter via high affinity FIS2/3sites, but on transition to stationary phase (12h), the FIS concentration subsides (see Fig 4a, lower panel) enabling transcription of pelD. Our data suggest that the burst of peID expression on transition of cells to stationary phase is likely facilitated by two additional mechanisms. First, the reduced transcription of div (e.g. due to the global relaxation of DNA), would abolish competition with pelD. Second, due to the increased CAMP levels at this stage [43], the cooperative binding of CAMP-CRP to CRP1 and CRP2 activator sites [26] would stabilise RNAP binding at pelD leading to its maximal expression. We propose that growth phase dependent competition between FIS and CRP 
binding in conjunction with alterations of DNA topology provide an efficient temporal mechanism enabling both tight repression and strong activation of the pelD promoter. It is likely that binding and bending of the DNA by CRP [48] at the CRP1 and CRP2 sites generally facilitates the recruitment of the RNAP molecules, whereas the changing pattern of CRP site occupation (binding of CRP at CRP1 initially, and then at both CRP1 and CRP2), determines the distribution of polymerase between div and pelD. This mechanism implies that inactivation of the div promoter, despite its potential to compete with the pelD promoter for RNAP binding, could lead to decreased pelD activity.

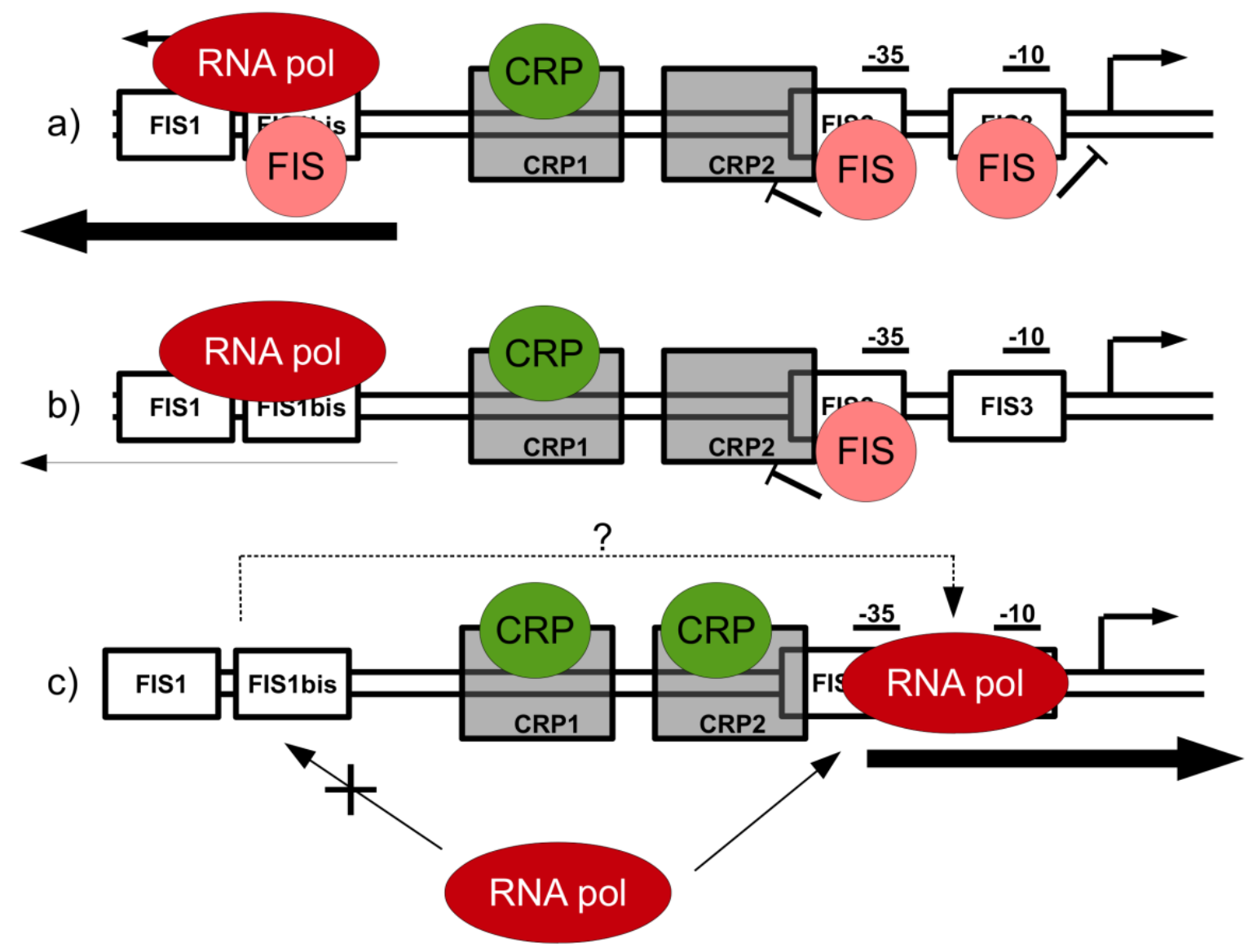

Fig 8: Proposed model for the temporal control of peID by FIS, CRP and the divergent promoter. For clarity, the peID regulatory region is represented in a linear fashion. Binding sites are represented by boxes (white for FIS, grey for CRP). Proteins are represented by ellipses: red for RNA polymerase, green for CRP, 
pink for FIS. Large horizontal arrows indicate transcription. Lines ending with a bar indicate inhibition. a) During early exponential phase, FIS sequentially occupies most of its binding sites and both the pelD and div promoters are repressed, albeit to different extent, since at this stage div is activated by high negative superhelicity. The div promoter efficiently binds RNA polymerase due to its high affinity for RNAP and the low affinity of the competitor FIS to the FIS1 and FIS1bis sites. Binding of CRP at CRP1 site also favours binding of polymerase at div and so, the RNAP is "parking" at div. b) During the late exponential phase, DNA relaxation inactivates div and presumably destabilises the binding of RNAP. At this stage, pelD is still repressed due to the overlapping high-affinity FIS sites. c) During transition to stationary phase FIS levels subside and peID is de-repressed. RNA polymerase now preferentially binds pelD. The activating effect of div on pelD at this stage is most likely due to a transfer of idling RNAP molecules previously "parking" at div towards pelD. This results in very strong transcription of pelD.

\section{Biological implications}

Our data strongly suggest that the growth phase dependent regulation of pelD is under the control of changing composition of higher-order nucleoprotein complexes formed in the pelD regulatory region during the growth cycle. Such "alteration" of the composition of regulatory nucleoprotein complexes during the growth cycle [49] seems to be a common design principle employed for the growth phase-dependent gene regulation. Similar regulatory mechanisms can be found not only at different promoters in single species but also in different species, whereby involvement of several FIS binding sites in promoter repression is not unusual. A well-known example is the negative autoregulation of the fis promoter involving numerous FIS binding sites [50], and there are other examples, such as the mom [36] and the gyr promoters of E. coli [6] and Salmonella [51], to name just a few. However, multiple FIS binding sites can also be used to exert either an activating or repressing effect [52].

On the other hand, there are only a few reports of a direct competition of FIS with 
611 an activator [53,54]. The case of the crp2 promoter is particularly relevant [54] as it shares 612 many similarities with the peID promoter such as two high-affinity FIS binding sites 613 overlapping the -10 and -35 elements, whereby the FIS site overlapping the -35 element 614 also competes with binding of the activator CRP. Moreover, the crp regulatory region 615 harbours also a FIS-regulated divergent promoter competing with the crp1 promoter, as 616 well as an upstream CRP binding site, which is little affected by FIS. Yet, there is no sequence homology between the $D$. dadantii pelD and $E$. coli crp promoters, suggesting a convergent evolution towards similar structural organisation of gene regulatory regions.

Also the E. coli fis promoter is subject to complex regulation involving FIS, CRP and a supercoiling-dependent divergent promoter [45]. In this latter case the RNAP molecule binding an upstream divergent site appears to directly interact with the RNAP bound at the fis promoter and this interaction is implicated in sensing the changing DNA superhelicity during the growth cycle. Similarly, in the peID regulatory region it is the div promoter which strongly depends on supercoiling and thus may serve as a sensor of DNA superhelicity transmitting its changes to the peID promoter. Whether the two RNAP molecules binding in the pelD regulatory region can interact, is an open question that merits further investigation. Theoretically, the 155 bp separation between the div and pelD transcription start sites (given that RNAP footprints are typically $70 \mathrm{bp}$ long) could suffice to avoid steric hindrance between the RNAP molecules, but assuming that polymerase on average wraps about $90 \mathrm{bp}$ of on binding the promoter DNA [55,56] there could be competition, as is for example, in the case of the crp promoter $([54,57]$ and Fig S6). It is noteworthy that also in the crp regulatory region, only one of the promoters (crp2) appears to be strongly sensitive to supercoiling, suggesting that the topological state of the DNA is one major variable involved in modulating the growth phase-dependent impacts of regulatory nucleoprotein 
complexes assembled on divergent promoter modules [54,57].

We infer that the organisation of pelD promoter region reveals a strategic design of protein binding sites evolved for reconciliation of strong expression with regulatability. The pelD gene is one among the many bacterial virulence genes known to be under the control of several TFs and be also tightly environmentally regulated [58]. Indeed, it is not uncommon to see more than five different TFs acting on a single virulence gene $[16,59,60]$. By taking into account the binding of CRP, FIS and RNA polymerase at multiple sites in peID, as well as many regulators unconsidered in this study, such as $\mathrm{H}-\mathrm{NS}$ and PecT, which are key components in the thermoregulation of the pel genes [61], or PecS, which is known to interact with CRP [26], a highly dynamic regulatory mechanism has to be envisioned. Comprehensive understanding of the strategic design underlying the spatial organisation of regulatory binding sites in pelD and the topological constraints imposed on the cooperative and antagonistic interactions between various TFs remains an important focus for further studies.

\section{FUNDING INFORMATION}

This work was supported by the Centre National de la Recherche Scientifique; Université de Lyon; and Institut National des Sciences Appliquées [BQR 2013 «Topologie de l'ADN et organisation du nucléoïde " to S.R.]. The funders had no role in study design, data collection and interpretation, or the decision to submit the work for publication.

\section{ACKNOWLEDGEMENT}

The authors would like to thank the teams of Agnès Rodrigue and Xavier Charpentier for 


\section{REFERENCES}

[1] D.F. Browning, S.J.W. Busby, The regulation of bacterial transcription initiation, Nat. Rev. Microbiol. 2 (2004) 57-65. doi:10.1038/nrmicro787.

[2] A. Bhat, M. Shin, J.-H. Jeong, H.-J. Kim, H.-J. Lim, J.H. Rhee, S.-Y. Paik, K. Takeyasu, T. Tobe, H. Yen, G. Lee, H.E. Choy, DNA looping-dependent autorepression of LEE1 P1 promoters by Ler in enteropathogenic Escherichia coli (EPEC), Proc. Natl. Acad. Sci. U. S. A. 111 (2014) E2586-E2595. doi:10.1073/pnas.1322033111.

[3] Z.-A. Ouafa, S. Reverchon, T. Lautier, G. Muskhelishvili, W. Nasser, The nucleoid-associated proteins H-NS and FIS modulate the DNA supercoiling response of the pel genes, the major virulence factors in the plant pathogen bacterium Dickeya dadantii, Nucleic Acids Res. 40 (2012) 4306-4319. doi:10.1093/nar/gks014.

[4] D. Skoko, D. Yoo, H. Bai, B. Schnurr, J. Yan, S.M. McLeod, J.F. Marko, R.C. Johnson, Mechanism of chromosome compaction and looping by the E. coli nucleoid protein Fis, J. Mol. Biol. 364 (2006) 777-798. doi:10.1016/j.jmb.2006.09.043.

[5] S.C. Dillon, C.J. Dorman, Bacterial nucleoid-associated proteins, nucleoid structure and gene expression, Nat. Rev. Microbiol. 8 (2010) 185-195. doi:10.1038/nrmicro2261.

[6] R. Schneider, A. Travers, T. Kutateladze, G. Muskhelishvili, A DNA architectural protein couples cellular physiology and DNA topology in Escherichia coli, Mol. Microbiol. 34 (1999) 953-964. doi:10.1046/j.13652958.1999.01656.X.

[7] D.J. Jin, C. Cagliero, Y.N. Zhou, Role of RNA Polymerase and Transcription in the Organization of the Bacterial Nucleoid, Chem. Rev. (2013) 130813134324001. doi:10.1021/cr4001429.

[8] A. Travers, G. Muskhelishvili, Bacterial chromatin, Curr. Opin. Genet. Dev. 15 (2005) 507-514. doi:10.1016/j.gde.2005.08.006.

[9] R. Schneider, A. Travers, G. Muskhelishvili, FIS modulates growth phase-dependent topological transitions of DNA in Escherichia coli, Mol. Microbiol. 26 (1997) 519-530.

[10] T. Ali Azam, A. Iwata, A. Nishimura, S. Ueda, A. Ishihama, Growth Phase-Dependent Variation in Protein Composition of the Escherichia coli Nucleoid, J. Bacteriol. 181 (1999) 6361-6370.

[11] D.F. Browning, D.C. Grainger, S.J. Busby, Effects of nucleoid-associated proteins on bacterial chromosome structure and gene expression, Curr. Opin. Microbiol. 13 (2010) 773-780. doi:10.1016/j.mib.2010.09.013.

[12] A. Duprey, S. Reverchon, W. Nasser, Bacterial virulence and Fis: adapting regulatory networks to the host environment, Trends Microbiol. 22 (2014) 92-99. doi:10.1016/j.tim.2013.11.008.

[13] B. Ma, M.E. Hibbing, H.-S. Kim, R.M. Reedy, I. Yedidia, J. Breuer, J. Breuer, J.D. Glasner, N.T. Perna, A. Kelman, A.O. Charkowski, Host Range and Molecular Phylogenies of the Soft Rot Enterobacterial Genera Pectobacterium and Dickeya, Phytopathology. 97 (2007) 1150-1163. doi:10.1094/PHYTO-97-9-1150.

[14] S. Hassan, V.E. Shevchik, X. Robert, N. Hugouvieux-Cotte-Pattat, PelN Is a New Pectate Lyase of Dickeya dadantii with Unusual Characteristics, J. Bacteriol. 195 (2013) 2197-2206. doi:10.1128/JB.02118-12.

[15] F. Tardy, W. Nasser, J. Robert-Baudouy, N. Hugouvieux-Cotte-Pattat, Comparative analysis of the five major Erwinia chrysanthemi pectate lyases: enzyme characteristics and potential inhibitors., J. Bacteriol. 179 (1997) 2503-2511.

[16] S. Reverchon, W. Nasser, Dickeya ecology, environment sensing and regulation of virulence programme: Dickeya dadantii pathogenicity, Environ. Microbiol. Rep. (2013) n/a-n/a. doi:10.1111/1758-2229.12073.

[17] S. Reverchon, W. Nasser, J. Robert-Baudouy, Characterization of kdgR, a gene of Erwinia chrysanthemi that regulates pectin degradation, Mol. Microbiol. 5 (1991) 2203-2216.

[18] W. Nasser, S. Reverchon, G. Condemine, J. Robert-Baudouy, Specific Interactions of Erwinia chrysanthemi KdgR Repressor with Different Operators of Genes Involved in Pectinolysis, J. Mol. Biol. 236 (1994) 427-440. doi:10.1006/jmbi.1994.1155.

[19] W. Nasser, J. Robert-Baudouy, S. Reverchon, Antagonistic effect of CRP and KdgR in the transcription control 
of the Erwinia chrysanthemi pectinolysis genes, Mol. Microbiol. 26 (1997) 1071-1082. doi:10.1046/j.13652958.1997.6472020.x.

[20] S. Reverchon, F. Van Gijsegem, G. Effantin, O. Zghidi-Abouzid, W. Nasser, Systematic targeted mutagenesis of the MarR/SlyA family members of Dickeya dadantii 3937 reveals a role for MfbR in the modulation of virulence gene expression in response to acidic pH, Mol. Microbiol. 78 (2010) 1018-1037. doi:10.1111/j.13652958.2010.07388.x.

[21] S. Reverchon, D. Expert, J. Robert-Baudouy, W. Nasser, The cyclic AMP receptor protein is the main activator of pectinolysis genes in Erwinia chrysanthemi., J. Bacteriol. 179 (1997) 3500-3508.

[22] T. Lautier, W. Nasser, The DNA nucleoid-associated protein Fis co-ordinates the expression of the main virulence genes in the phytopathogenic bacterium Erwinia chrysanthemi, Mol. Microbiol. 66 (2007) 1474-1490. doi:10.1111/j.1365-2958.2007.06012.x.

[23] C. Beaulieu, M. Boccara, F. Van Gijsegem, Pathogenic Behavior of Pectinase-Defective Erwinia chrysanthemi Mutants on Different Plants, Mol. Plant. Microbe Interact. 6 (1993) 197. doi:10.1094/MPMI-6-197.

[24] M. Boccara, A. Diolez, M. Rouve, A. Kotoujansky, The role of individual pectate lyases of Erwinia chrysanthemi strain 3937 in pathogenicity on saintpaulia plants, Physiol. Mol. Plant Pathol. 33 (1988) 95-104. doi:10.1016/0885-5765(88)90046-X.

[25] T. Lautier, N. Blot, G. Muskhelishvili, W. Nasser, Integration of two essential virulence modulating signals at the Erwinia chrysanthemi pel gene promoters: a role for Fis in the growth-phase regulation, Mol. Microbiol. 66 (2007) 1491-1505. doi:10.1111/j.1365-2958.2007.06010.x.

[26] C. Rouanet, K. Nomura, S. Tsuyumu, W. Nasser, Regulation of pelD and pelE, Encoding Major Alkaline Pectate Lyases in Erwinia chrysanthemi: Involvement of the Main Transcriptional Factors, J. Bacteriol. 181 (1999) 5948-5957.

[27] Miller JH, Experiment in Molecular Genetics, Cold Spring Harbor Laboratory Press, 1972.

[28] A. Duprey, V. Chansavang, F. Fremion, C. Gonthier, Y. Louis, P. Lejeune, F. Springer, V. Desjardin, A. Rodrigue, C. Dorel, "NiCo Buster": engineering E. coli for fast and efficient capture of cobalt and nickel, J. Biol. Eng. 8 (2014) 19. doi:10.1186/1754-1611-8-19.

[29] C. Marschall, V. Labrousse, M. Kreimer, D. Weichart, A. Kolb, R. Hengge-Aronis, Molecular analysis of the regulation of csiD, a carbon starvation-inducible gene in Escherichia coli that is exclusively dependent on $\sigma \mathrm{S}$ and requires activation by cAMP-CRP, J. Mol. Biol. 276 (1998) 339-353. doi:10.1006/jmbi.1997.1533.

[30] J. Sambrook, D.W. Russell, Molecular cloning : a laboratory manual, 3rd ed., Cold Spring Harbor Laboratory Press, 2001.

[31] H. De Jong, C. Ranquet, D. Ropers, C. Pinel, J. Geiselmann, Experimental and computational validation of models of fluorescent and luminescent reporter genes in bacteria, BMC Syst. Biol. 4 (2010) 55.

[32] V.V. Verkhusha, I.M. Kuznetsova, O.V. Stepanenko, A.G. Zaraisky, M.M. Shavlovsky, K.K. Turoverov, V.N. Uversky, High Stability of Discosoma DsRed As Compared to Aequorea EGFP $\uparrow$, Biochemistry (Mosc.). 42 (2003) 7879-7884. doi:10.1021/bi034555t.

[33] N. Bardonnet, A. Trautwetter, G. Couchoux-Luthaud, C. Blanco, Plasmids with the uidA reporter gene for the detection of promoters and transcription signals, Mol. Gen. Genet. MGG. 212 (1988) 390-392.

[34] A. Hatoum, J. Roberts, Prevalence of RNA polymerase stalling at Escherichia coli promoters after open complex formation, Mol. Microbiol. 68 (2008) 17-28. doi:10.1111/j.1365-2958.2008.06138.x.

[35] R. Münch, K. Hiller, A. Grote, M. Scheer, J. Klein, M. Schobert, D. Jahn, Virtual Footprint and PRODORIC: an integrative framework for regulon prediction in prokaryotes, Bioinformatics. 21 (2005) 4187-4189. doi:10.1093/bioinformatics/bti635.

[36] S. Karambelkar, G. Swapna, V. Nagaraja, Silencing of toxic gene expression by Fis, Nucleic Acids Res. 40 (2012) 4358-4367. doi:10.1093/nar/gks037.

[37] W. Nasser, C. Dorel, J. Wawrzyniak, F. Van Gijsegem, M.-C. Groleau, E. Déziel, S. Reverchon, Vfm a new quorum sensing system controls the virulence of Dickeya dadantii, Environ. Microbiol. 15 (2013) 865-880. doi:10.1111/1462-2920.12049.

[38] V. James, N. Hugouvieux-Cotte-Pattat, Regulatory systems modulating the transcription of the pectinase genes of Erwinia chrysanthemi are conserved in Escherichia coli, Microbiol. Read. Engl. 142 ( Pt 9) (1996) 2613-2619.

[39] H.S. Kim, Cloning and Expression of $\beta$-Glucuronidase from Lactobacillus brevis in E. coli and Application in Bioconversion of Baicalin and Wogonoside, J. Microbiol. Biotechnol. 19 (2009) 1650-1655. doi:10.4014/jmb.0904.04053.

[40] J. Eriksson, T. Nordström, P. Nyrén, Method enabling firefly luciferase-based bioluminometric assays at elevated temperatures, Anal. Biochem. 314 (2003) 158-161. doi:10.1016/S0003-2697(02)00647-4.

[41] W.D. Kepseu, J.-A. Sepulchre, S. Reverchon, W. Nasser, Toward a quantitative modeling of the synthesis of the pectate lyases, essential virulence factors in Dickeya dadantii, J. Biol. Chem. 285 (2010) 28565-28576. doi:10.1074/jbc.M110.114710. 
[42] A. Maxwell, DNA gyrase as a drug target, Trends Microbiol. 5 (1997) 102-109. doi:10.1016/S0966842X(96)10085-8.

[43] V.L. Balke, J.D. Gralla, Changes in the linking number of supercoiled DNA accompany growth transitions in Escherichia coli, J. Bacteriol. 169 (1987) 4499-4506.

[44] F. Rojo, Mechanisms of transcriptional repression, Curr. Opin. Microbiol. 4 (2001) 145-151. doi:10.1016/S13695274(00)00180-6.

[45] V. Gerganova, S. Maurer, L. Stoliar, A. Japaridze, G. Dietler, W. Nasser, T. Kutateladze, A. Travers, G. Muskhelishvili, Upstream Binding of Idling RNA Polymerase Modulates Transcription Initiation from a Nearby Promoter, J. Biol. Chem. (2015) jbc.M114.628131. doi:10.1074/jbc.M114.628131.

[46] S.M. McLeod, S.E. Aiyar, R.L. Gourse, R.C. Johnson, The C-terminal domains of the RNA polymerase $\alpha$ subunits: contact site with fis and localization during co-activation with CRP at the Escherichia coli proP P2 promoter, J. Mol. Biol. 316 (2002) 517-529. doi:10.1006/jmbi.2001.5391.

[47] P.R. Davidsson, T. Kariola, O. Niemi, E.T. Palva, Pathogenicity of and plant immunity to soft rot pectobacteria, Front. Plant Sci. 4 (2013). doi:10.3389/fpls.2013.00191.

[48] S.-H. Lin, J.C. Lee, Determinants of DNA Bending in the DNA-Cyclic AMP Receptor Protein Complexes in Escherichia coli, Biochemistry (Mosc.). 42 (2003) 4809-4818. doi:10.1021/bi027259+.

[49] W. Nasser, R. Schneider, A. Travers, G. Muskhelishvili, CRP Modulates fis Transcription by Alternate Formation of Activating and Repressing Nucleoprotein Complexes, J. Biol. Chem. 276 (2001) 17878-17886. doi:10.1074/jbc.M100632200.

[50] C.A. Ball, R. Osuna, K.C. Ferguson, R.C. Johnson, Dramatic changes in Fis levels upon nutrient upshift in Escherichia coli., J. Bacteriol. 174 (1992) 8043-8056.

[51] O.M. Keane, C.J. Dorman, The gyr genes of Salmonella enterica serovar Typhimurium are repressed by the factor for inversion stimulation, Fis, Mol. Genet. Genomics. 270 (2003) 56-65. doi:10.1007/s00438-003-0896-1.

[52] A. Chastanet, R. Losick, Just-in-Time Control of Spo0A Synthesis in Bacillus subtilis by Multiple Regulatory Mechanisms $\nabla$, J. Bacteriol. 193 (2011) 6366-6374. doi:10.1128/JB.06057-11.

[53] D.J.P. Squire, M. Xu, J.A. Cole, S.J.W. Busby, D.F. Browning, Competition between NarL-dependent activation and Fis-dependent repression controls expression from the Escherichia coli yeaR and ogt promoters, Biochem. J. 420 (2009) 249-257. doi:10.1042/BJ20090183.

[54] G. González-Gil, R. Kahmann, G. Muskhelishvili, Regulation of crp transcription by oscillation between distinct nucleoprotein complexes, EMBO J. 17 (1998) 2877-2885.

[55] C. Rivetti, M. Guthold, C. Bustamante, Wrapping of DNA around the E.coli RNA polymerase open promoter complex, EMBO J. 18 (1999) 4464-4475. doi:10.1093/emboj/18.16.4464.

[56] S. Maurer, J. Fritz, G. Muskhelishvili, A. Travers, RNA polymerase and an activator form discrete subcomplexes in a transcription initiation complex, EMBO J. 25 (2006) 3784-3790. doi:10.1038/sj.emboj.7601261.

[57] A. Hanamura, H. Aiba, Molecular mechanism of negative autoregulation of Escherichia coli crp gene., Nucleic Acids Res. 19 (1991) 4413-4419.

[58] J.S. Kroll, Bacterial virulence: an environmental response., Arch. Dis. Child. 66 (1991) 361-363.

[59] C. Altier, Genetic and environmental control of Salmonella invasion, J Microbiol. 43 (2005) 85-92.

[60] T.L. Yahr, M.C. Wolfgang, Transcriptional regulation of the Pseudomonas aeruginosa type III secretion system, Mol. Microbiol. 62 (2006) 631-640. doi:10.1111/j.1365-2958.2006.05412.x.

[61] E. Hérault, S. Reverchon, W. Nasser, Role of the LysR-type transcriptional regulator PecT and DNA supercoiling in the thermoregulation of pel genes, the major virulence factors in Dickeya dadantii: Dickeya dadantii PecT protein and virulence thermoregulation, Environ. Microbiol. 16 (2014) 734-745. doi:10.1111/1462-2920.12198.

[62] A. Kotoujansky, M. Lemattre, P. Boistard, Utilization of a thermosensitive episome bearing transposon TN10 to isolate Hfr donor strains of Erwinia carotovora subsp. chrysanthemi., J. Bacteriol. 150 (1982) 122-131.

[63] G. González-Gil, P. Bringmann, R. Kahmann, FIS is a regulator of metabolism in Escherichia coli, Mol. Microbiol. 22 (1996) 21-29. doi:10.1111/j.1365-2958.1996.tb02652.x.

[64] W.G. Miller, J.H. Leveau, S.E. Lindow, Improved gfp and inaZ broad-host-range promoter-probe vectors, Mol. Plant-Microbe Interact. MPMI. 13 (2000) 1243-1250. doi:10.1094/MPMI.2000.13.11.1243. 
a)

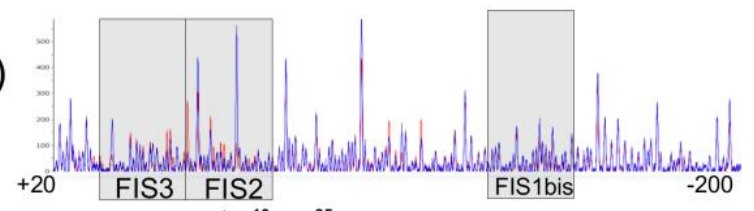

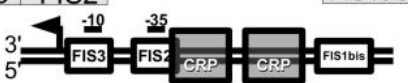

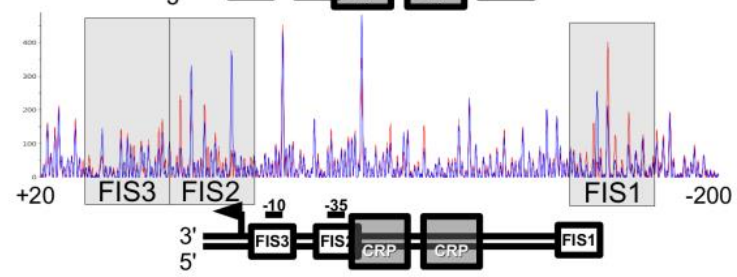

b)

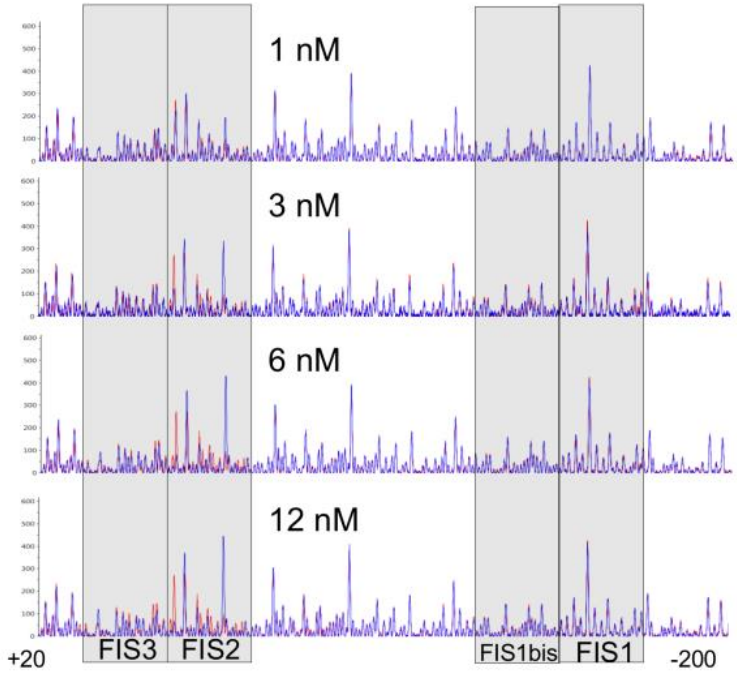

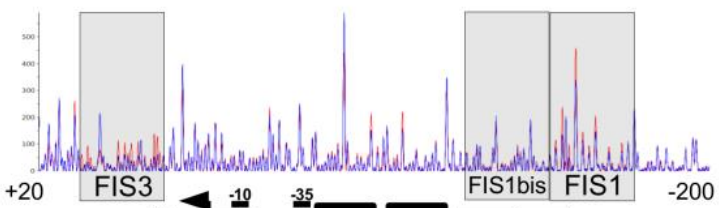
+20 FIS3 3 - 10 FIS1bis FIS1 -200
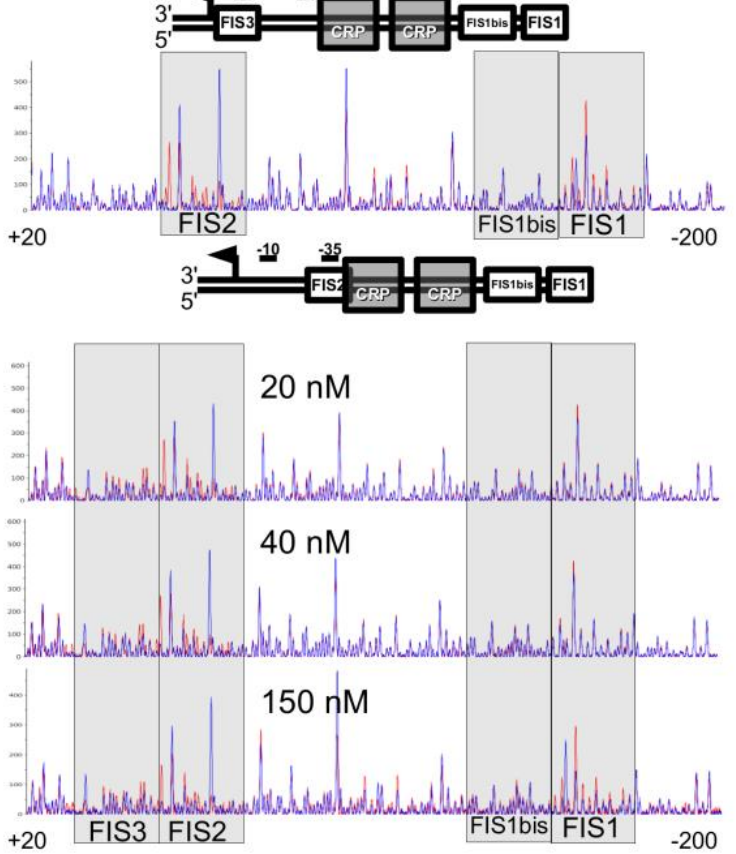

Fig S1: DNase I footprinting of FIS binding in the peID regulatory region. DNase I footprinting was performed using fluorescently labelled linear peID DNA. The profiles represent the pattern obtained for the template strand. The red and the blue profiles correspond respectively to the samples without and with FIS. a) Profiles for peID DNA samples with inactivated FIS1, FIS1bis, FIS2 or FIS3 sites at 150 nM FIS concentration. b) Profiles of the wild type peID DNA incubated with increasing concentrations of FIS (from 1 to $150 \mathrm{nM})$ 


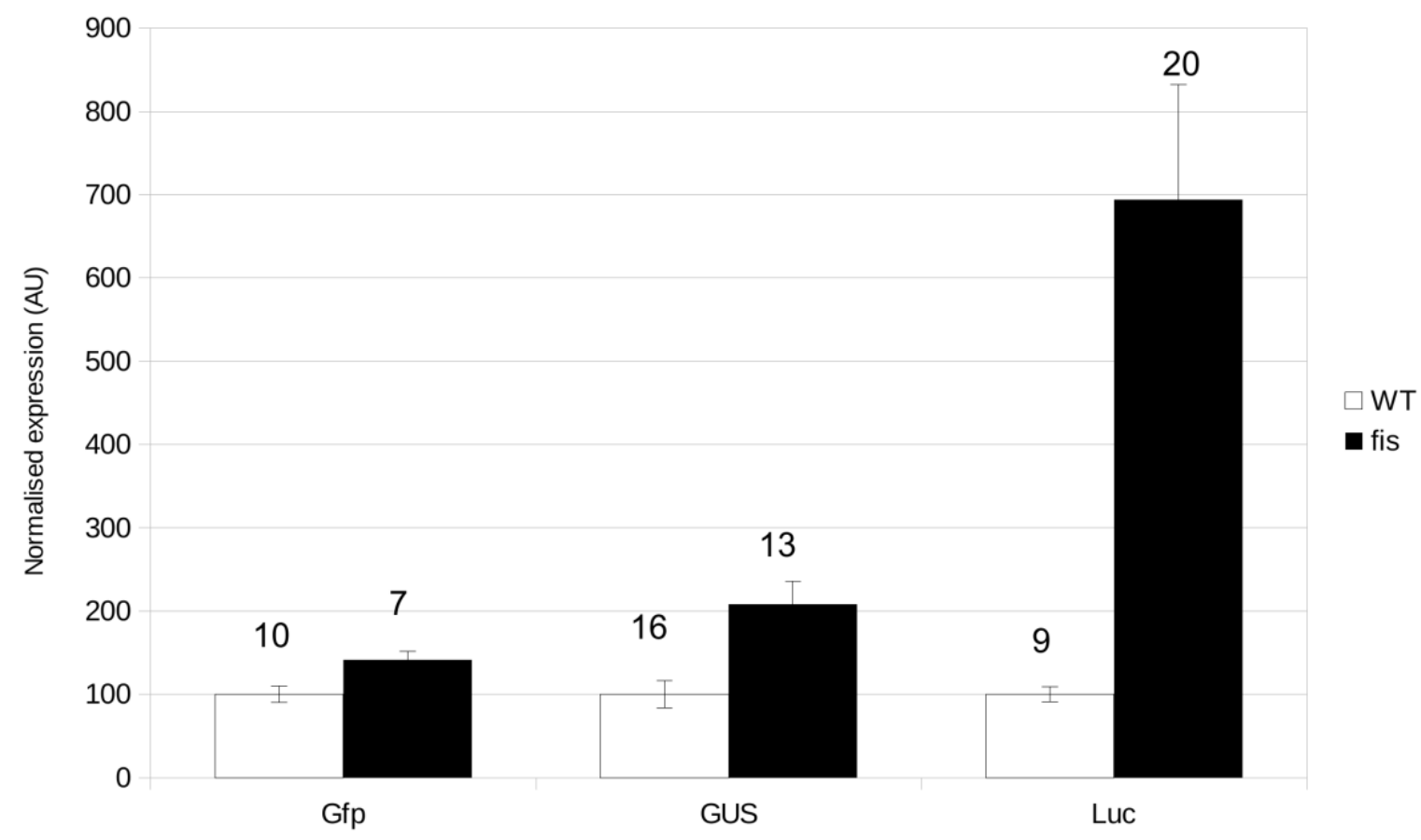

828 Fig S2: Comparison between the Gfp and Luc reporter systems in $\boldsymbol{D}$. dadantii and the GUS system in

829 E. coli. Bacteria transformed with the wild-type pelD regulatory region in a reporter plasmid were cultured in 830 equivalent conditions (see materials and methods). The normalised fluorescence and GUS specific activity 831 were taken in early stationary phase and the luminescence at the maximum. Data represents the mean of 3 832 (GUS) to 12 replicates (Gfp and Luc) and error bars represent the standard deviation. Numbers above the 833 error bars indicate the coefficient of variation (in \%). 


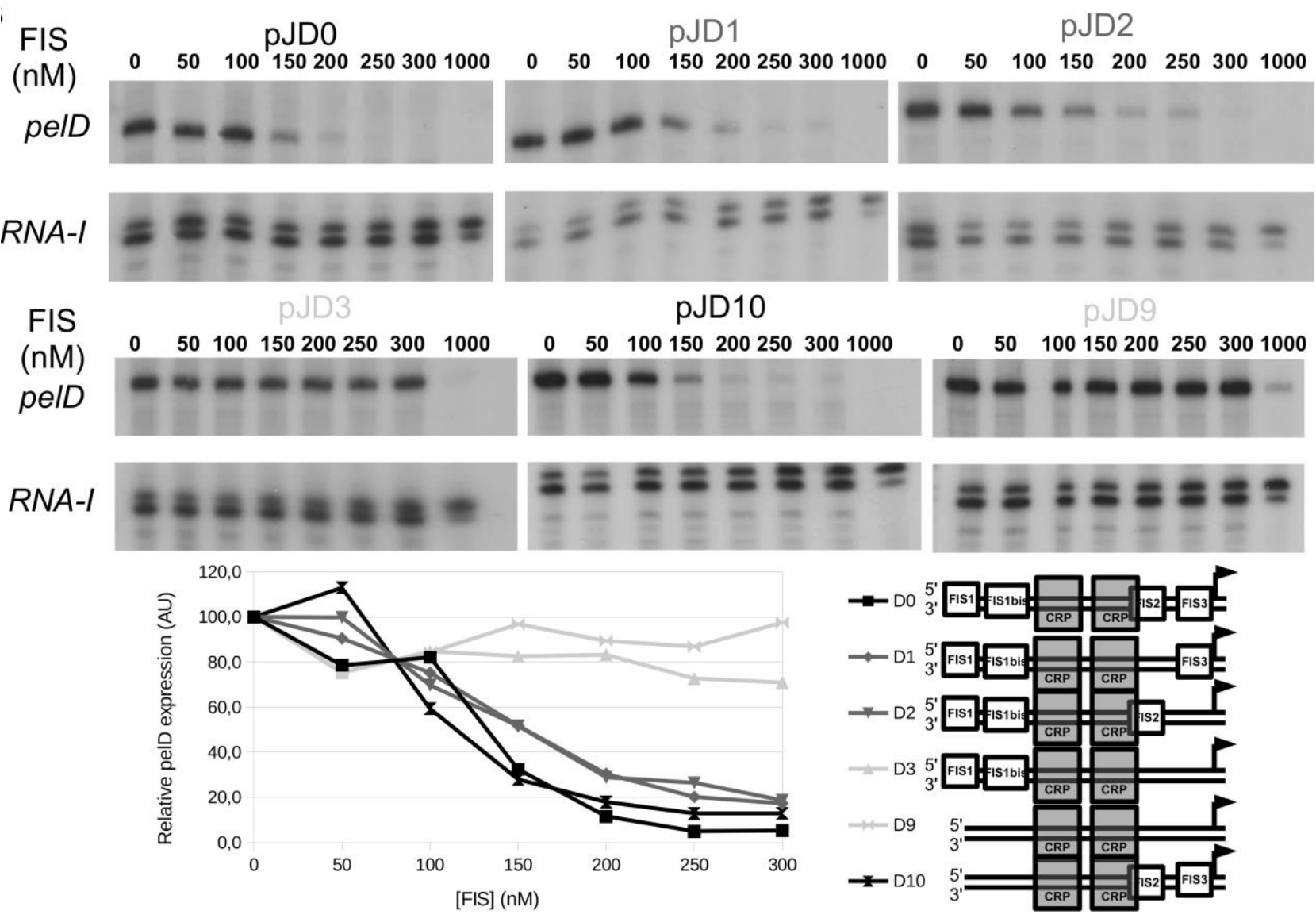

Fig S3: Effect of FIS binding on peID transcription in vitro. In vitro transcription was performed using several pelD constructs (plasmids D0, D1, D2, D3, D9 and D10 indicated in the bottom left panel) in presence of $100 \mathrm{nM}$ CRP, $40 \mathrm{nM}$ RNA polymerase and varying concentrations of FIS. The RNA-I internal control was used as a standard for quantification of the pelD transcript (bottom right panel). 

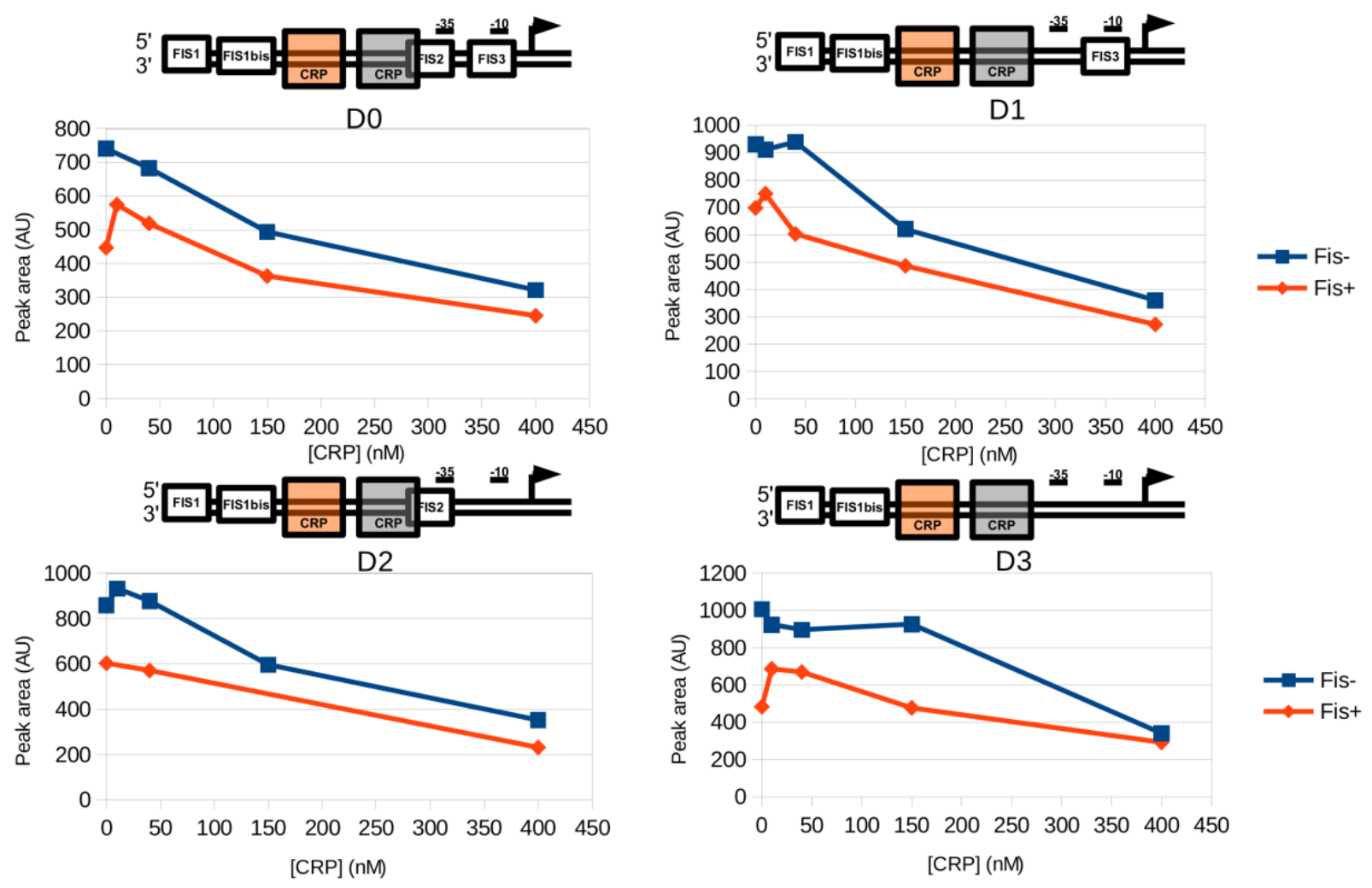

Fig S4: Absence of modification of the CRP1 DNase I footprint by the presence of FIS. A quantitative DNase I footprint was performed on fluorescently labelled pelD regulatory regions, in identical conditions as in figure 5. The area of the peaks at one position inside CRP1 (at position -85 relatively to the transcription start site) for different CRP concentrations is represented. 
a)

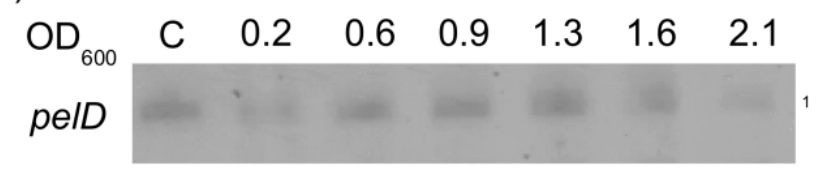

c)

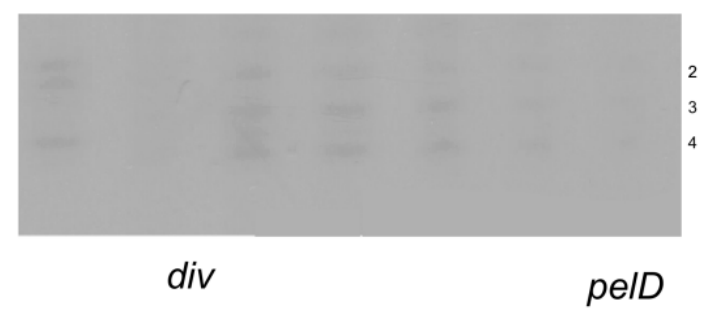

5' ACATATAGCTGAA

3' $\underset{4}{\text { TGTATATCGACTT }}$ b)

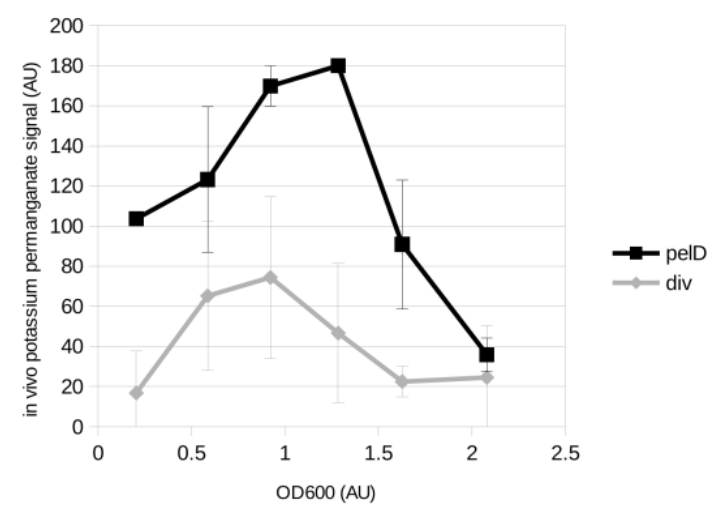

847 Fig S5: in vivo potassium permanganate footprinting of the pelD regulatory region. pKD0 (wild-type peID) was transformed into $D$. dadantii. Bacteria were then cultured in M63S + PGA and an in vivo potassium permanganate footprinting was performed as described in materials and methods. (a) image of one replicate of the experiment. Lane $\mathrm{C}$ corresponds to an in vitro potassium permanganate footprinting control performed on the same plasmid with $100 \mathrm{nM}$ CRP and $40 \mathrm{nM}$ RNAP. Bands are numbered and localised in part c of the figure. (b) quantification of the intensity of the bands, represented as mean $+/-$ SD of 2 independent biological replicates. (c) Position of the attacked nucleotides of part a. Both strands are shown and transcription start sites are underlined. 


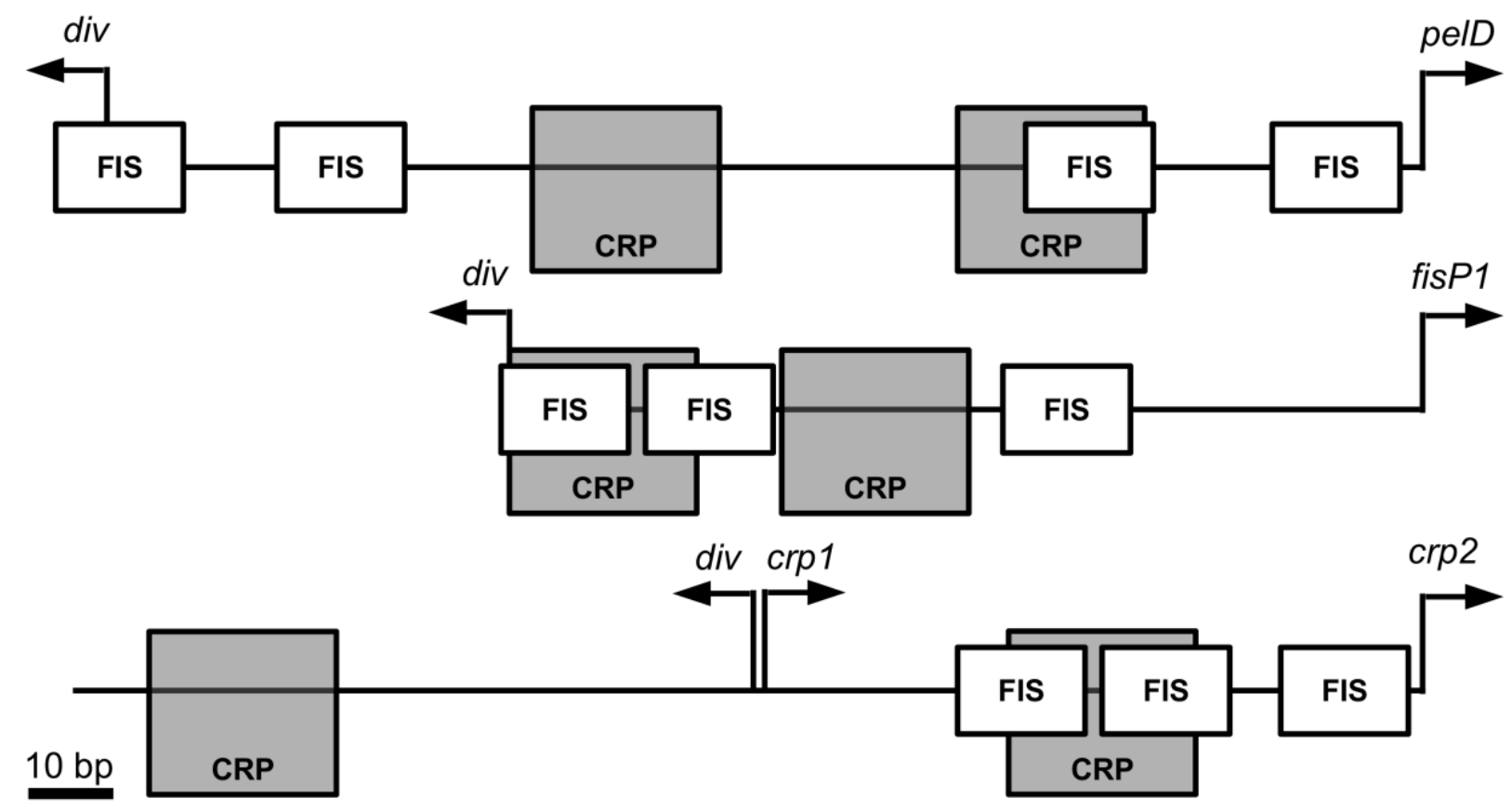

856 Fig S6: Regulatory structure of 3 different relevant promoters regulated by a divergent promoter. The 857 pelD (D. dadantii), fis (E. coli) and $\operatorname{crp~(E.~coli)~regulatory~regions~are~represented.~The~known~promoters~are~}$ 858 represented with an arrow, FIS sites by white boxes and CRP sites by gray boxes, as described in the 859 litterature and this work. The promoter which is coupled to div is drawn on the right. All regulatory regions 860 and binding sites are represented to scale. The scale bar in the bottom left corner represents 10 base pairs.

Table S1: strains, plasmids and oligos used in this study

\begin{tabular}{|c|c|c|c|}
\hline Strains & Description & & Ref \\
\hline \multicolumn{4}{|l|}{ D. dadantii } \\
\hline 3937 (A4922) & Wild-type strain isolated from Saintpaulia ionantha & & [62] \\
\hline A4474 & 3937 fis:: $\mathrm{Cm}^{\mathrm{R}}$ & & [22] \\
\hline \multicolumn{4}{|l|}{ E. coli } \\
\hline CSH50 & F- araBAD-0 M(pro-lac) $\lambda$-rpsL-(strR) thi- fimE1::IS1- & & [27] \\
\hline 13506 & CSH50 fis:: $\mathrm{Cm}^{\mathrm{R}}$ & & [63] \\
\hline Plasmids & Description & $\begin{array}{l}\text { Inactivated } \\
\text { FIS sites }\end{array}$ & Ref \\
\hline pNB4 & Reporter vector with uid $A, \mathrm{AmpR}$ & & [33] \\
\hline pProbe-AT ' & Reporter vector with $g f p, A m p R$ & & [64] \\
\hline
\end{tabular}


pBluescript SK+

Cloning vector, AmpR

pBluescript KS+

pUCter-Luc-Cm

pJV

pWN2481 (pKD0)

pKD1

pKD2

pKD3

pKD4

pKD5

pKD7

pKD9

pKD10

pKD24

pKD26

pKD27

pKD28

pND0

pLD0

pPDO

pPD1

pPD2

pPD3

pPD4

pPD5

pPD7

pPD9

pPD24

pPD26

pPD27

pPD28

pJD0

pJD1

pJD2

pJD3

pJD9

pJD10

pJD24

pID0

pID24

Cloning vector, AmpR div inactivated CRP1 inactivated

pProbe-AT inactivated and CRP2 inactivated
Reporter vector with luc, AmpR CmR

In vitro transcription vector $\mathrm{AmpR}$

pBluescript KS+ containing the wild-type peID regulatory region between sites EcoRI and HindIII

Stratagene

Stratagene

(7)

This work

[26]

pBluescript SK+ containing a mutated pelD regulatory region at site Smal ( $T 7$ promoter oriented)

2

3

pBluescript SK+ containing a mutated peID regulatory region at site Smal (T7 promoter oriented)

2,3

pBluescript SK+ containing a mutated peID regulatory region at site Smal (T7 promoter oriented)

pBluescript $\mathrm{SK}+$ containing a mutated pelD regulatory region at site Smal (T7 promoter oriented)

pBluescript $\mathrm{SK}+$ containing a mutated pelD regulatory region at site Smal (T7 promoter oriented)

$1,2,3$

This work

This work

This work

1 bis

pBluescript KS+ containing a mutated peID regulatory region between sites EcoRI and HindIII

pBluescript SK+ containing a mutated pelD regulatory region at site Smal (T7 promoter oriented)

pBluescript $\mathrm{KS}+$ containing a mutated peID regulatory region between sites EcoRI and HindIII

1, 1 bis, 2, 3 This work

1,1 bis This work

pBluescript KS+ containing a mutated peID regulatory region between sites EcoRI and HindIII, with none

pBluescript KS+ containing a mutated pelD regulatory region between sites EcoRI and HindIII, with none CRP2 inactivated

pBluescript KS+ containing a mutated pelD regulatory region between sites EcoRI and HindlII, with none

pBluescript KS+ containing a mutated peID regulatory region between sites EcoRI and HindIII, with none CRP1 and CRP2 inactivated

pNB4 containing the wild-type peID regulatory region between sites EcoRI and HindIII

none

pUCter-Luc-Cm containing the wild-type peID regulatory region between sites Nhel and Xhol

none

none

2

pProbe-AT ' containing a mutated pelD regulatory region between sites EcoRI and HindIII

3

pProbe-AT ' containing a mutated pelD regulatory region between sites EcoRI and HindIII

2,3

$1,2,3$

1 bis, 2,3

1 bis

1, 1bis, 2, 3 This work

pProbe-AT ' containing a mutated peID regulatory region between sites EcoRI and HindIII

none

pProbe-AT ' containing a mutated peID regulatory region between sites EcoRI and HindIII, with CRP2 none

pProbe-AT ' containing a mutated peID regulatory region between sites EcoRI and HindIII, with CRP1 none inactivated

pProbe-AT ' containing a mutated peID regulatory region between sites EcoRI and HindIII, with CRP1 none

pJV containing the wild-type peID regulatory region between sites EcoRI and Sall

none

pJV containing a mutated peID regulatory region between sites EcoRI and Sall

2

pJV containing a mutated peID regulatory region between sites EcoRI and Sall

pJV containing a mutated peID regulatory region between sites EcoRI and Sall

2,3

This work

pJV containing a mutated peID regulatory region between sites EcoRI and Sall

1, 1 bis, 2,3

1,1 bis

none

none

pProbe-AT ' containing a wild-type pelD regulatory region between sites $\mathrm{Kpnl}$ and $\mathrm{BamHI}$

pProbe-AT ' containing a mutated pelD regulatory region between sites $\mathrm{Kpnl}$ and $\mathrm{BamHI}$, with div none
This work

This work

This work

This work

This work

This work

This work

This work

This work

This work

This work

This work

This work

This work

This work

This work

This work

This work

This work

This work

This work

This work

This work
This work 
inactivated

\begin{tabular}{|c|c|c|}
\hline pID26 & $\begin{array}{l}\text { pProbe-AT ' containing a mutated pelD regulatory region between sites } \mathrm{Kpnl} \text { and } \mathrm{BamHI} \text {, with CRP2 none } \\
\text { inactivated }\end{array}$ & This work \\
\hline pID27 & $\begin{array}{l}\text { pProbe-AT ' containing a mutated pelD regulatory region between sites } \mathrm{Kpnl} \text { and } \mathrm{BamHI} \text {, with CRP1 none } \\
\text { inactivated }\end{array}$ & This work \\
\hline pID28 & $\begin{array}{l}\text { pProbe-AT ' containing a mutated pelD regulatory region between sites } \mathrm{Kpnl} \text { and } \mathrm{BamHI} \text {, with CRP1 none } \\
\text { and CRP2 inactivated }\end{array}$ & This work \\
\hline Primers & & Ref \\
\hline DNase pel FW & HEX-ACTATAGGGCGAATTGG & This work \\
\hline DNase pel REV & FAM-AATTAACССTCACTAAAGG & This work \\
\hline B0014 FW & AACAGCTGTCACACTGGCTCACСTTC & This work \\
\hline B0014 REV & AAGAATTCAATAATAAAAAAGCCGGATTAATAATC & This work \\
\hline bla 3B4 fluo & HEX-CAGGAAGGCAAAATGCCGC & This work \\
\hline pelDfis1bismut FW & GCTGAATTTAAAAtAAAAATTAATTCAAtATTCATAACTAAAAG & This work \\
\hline pelDfis1bismut REV & CTTTTAGTTATGAATaTTGAATTAATTTTTTATTTTAAATTCAGC & This work \\
\hline pelDFis1mutFW & TTTCAGATAAAAACtCTTATACATATAGtTGAATTTAAAA & This work \\
\hline pelDFis1mutREV & TTTTAAATTCAаCTATATGTATAAGaGTTTTTATCTGAAA & This work \\
\hline 35rev v2 FW & GAATTTAAAAGAAAAATTAATTCttCATTCATAACTAAAAGTTACC & This work \\
\hline 35rev v2 REV & GGTAACTTTTAGTTATGAATGaaGAATTAATTTTTTCTTTTAAATTC & This work \\
\hline 300 & ACTATAGGGCGAATTGG & This work \\
\hline 395 & CATAAAAAAAACGAGATTTTGAagtCAAAATAAACAATCGAAAACGC & This work \\
\hline 396 & GCGTTTTCGATTGTTTATTTTGactTCAAAATCTCGTTTTTTTTATG & This work \\
\hline 397 & CTAAAAGTTACCGGTCACGAagtCACTTTAGATAAAATTAATTAGC & This work \\
\hline 398 & GCTAATTAATTTTAATCTAAAGTGactTCGTGACCGGTAACTTTTAG & This work \\
\hline
\end{tabular}

862 


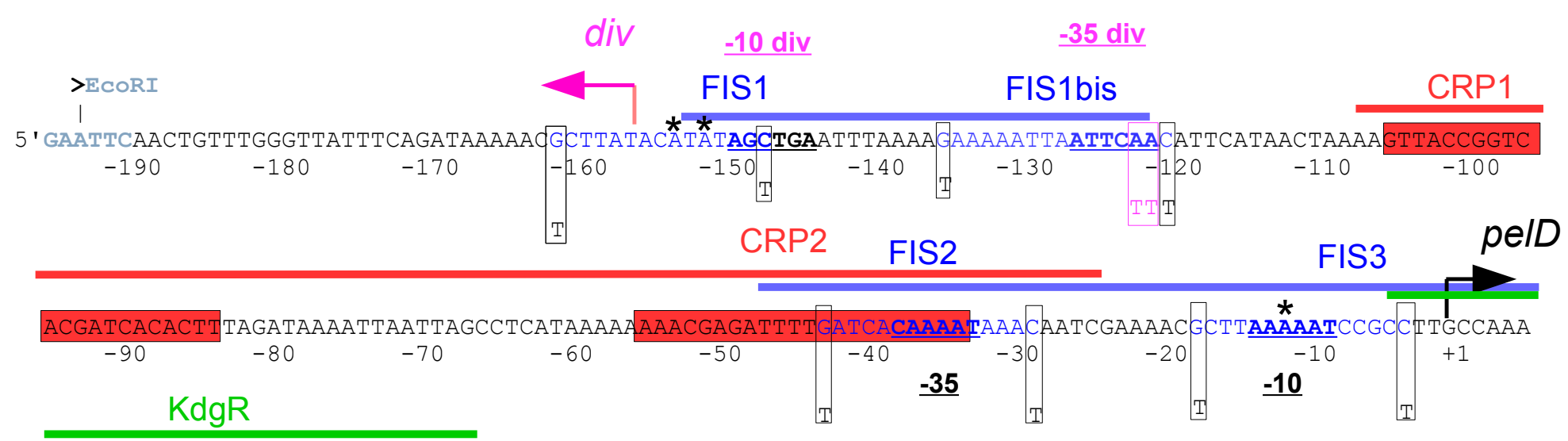

GGACAAAATGGCGTTTCATTTTTTTCACAAACACTTTTCAGTCAACAAAATTGGATTAGCGCAGATAGCGCAAGGAACCAGTCTATGAACAACACACGAG
10
20
$40 \quad 50$
60
$\begin{array}{ll}70 & 80\end{array}$
Start
100

$>$ HindIII

$\begin{array}{ccc}\text { TGTCTTCCGTAGGTACCAAAAGCTT } & \mathbf{3} \\ 110 & 120 & 130\end{array}$

130 


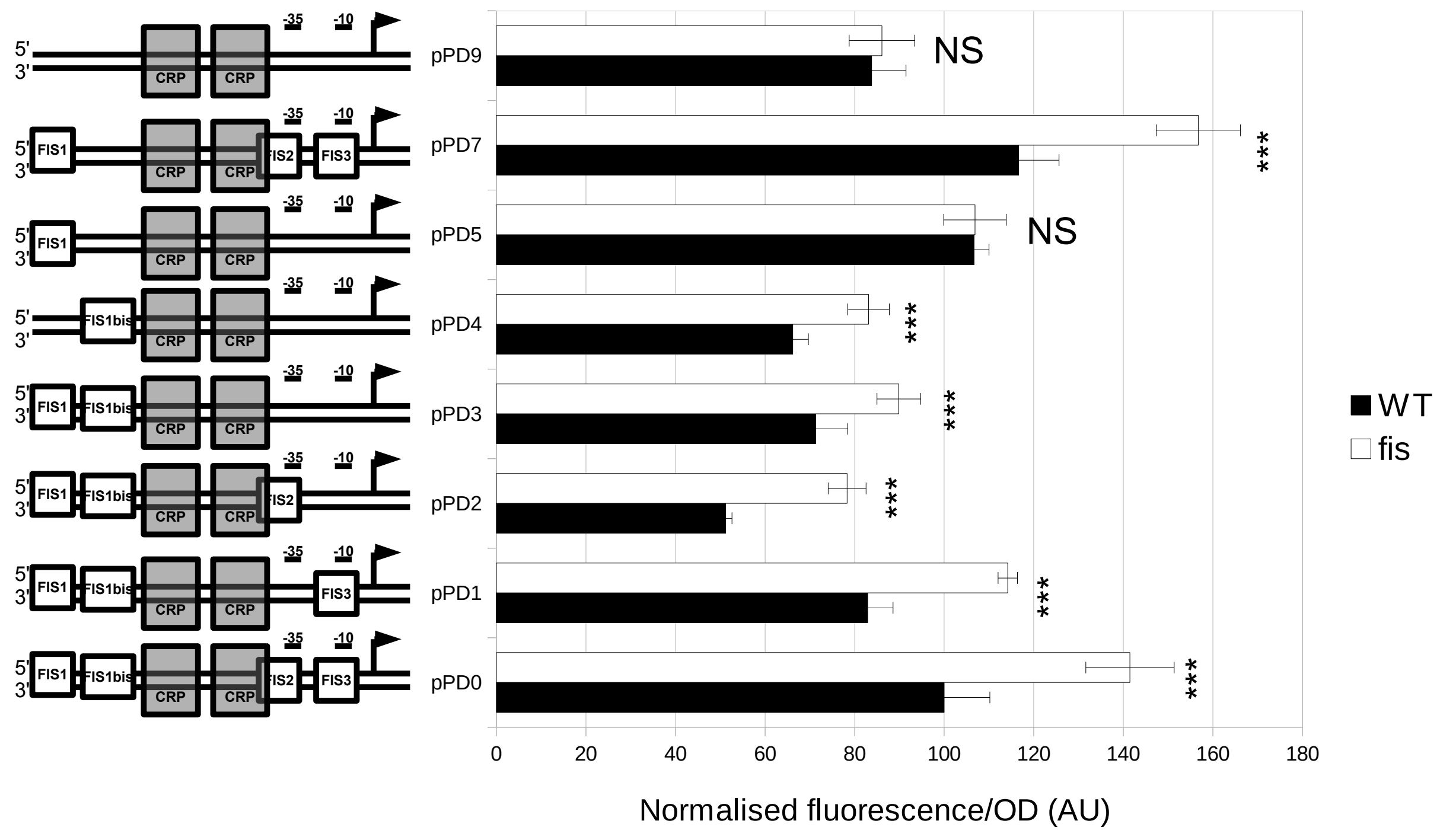



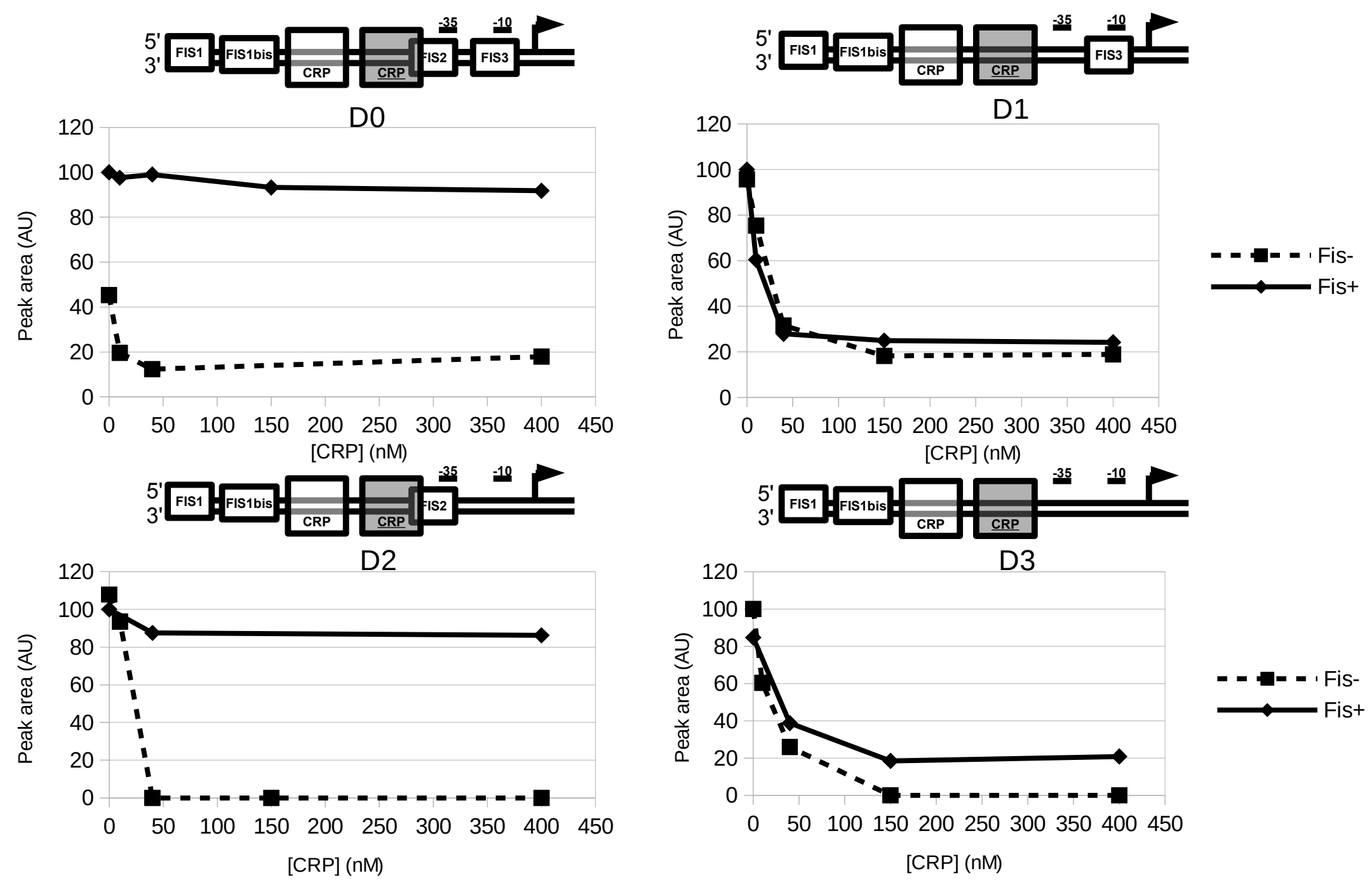
a)

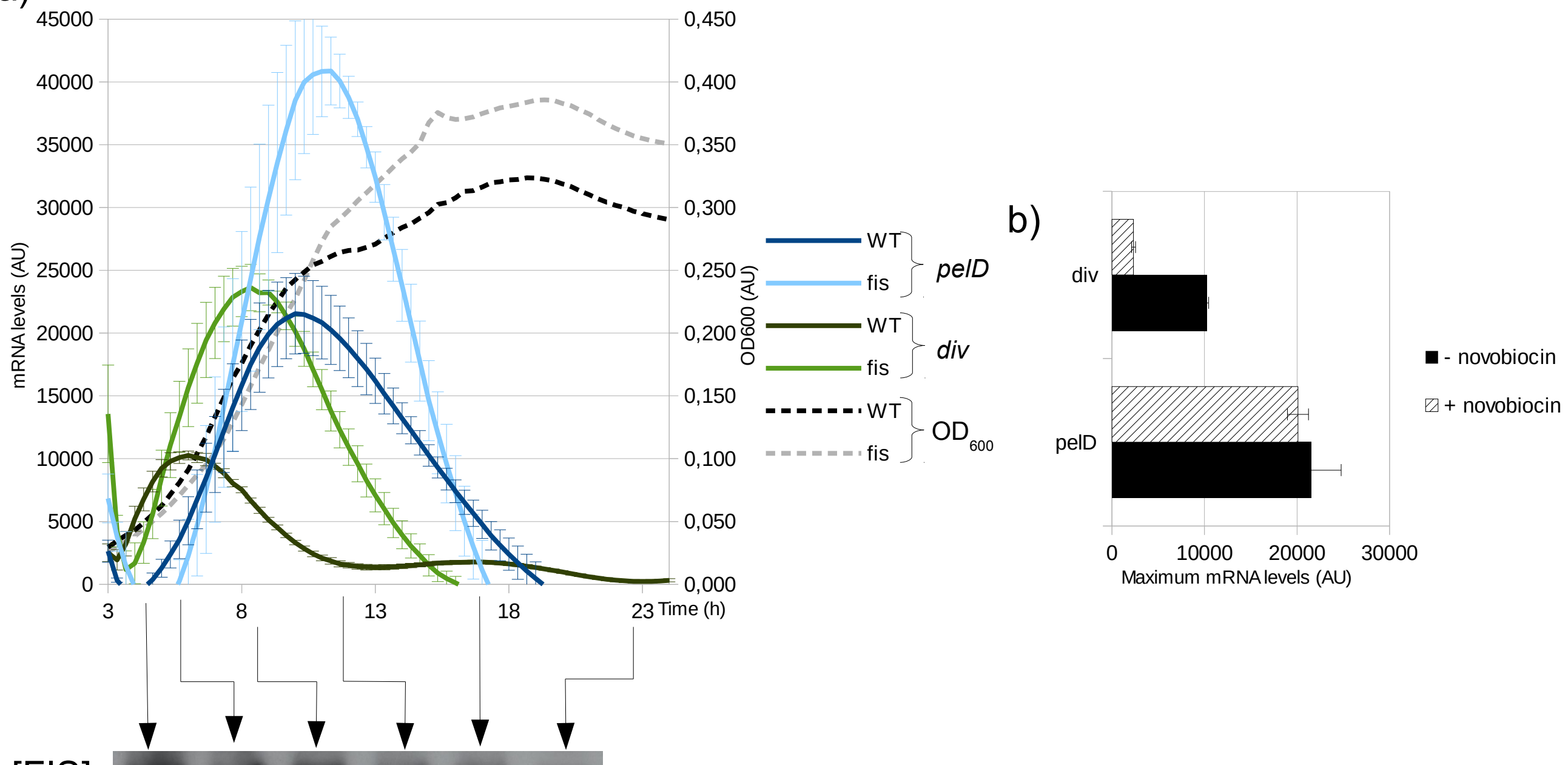

[FIS] 
a)

CRP1 GTTACCGGTCACGATCACACTT inactivated GTTACCGGTCACGAagtCACTT

CRP2 AAACGAGATTTTGATCACAAAA inactivated AAACGAGATTTTGAagtCAAAA consensus nnnTGTGAnnnnnnTCACAnnn b)

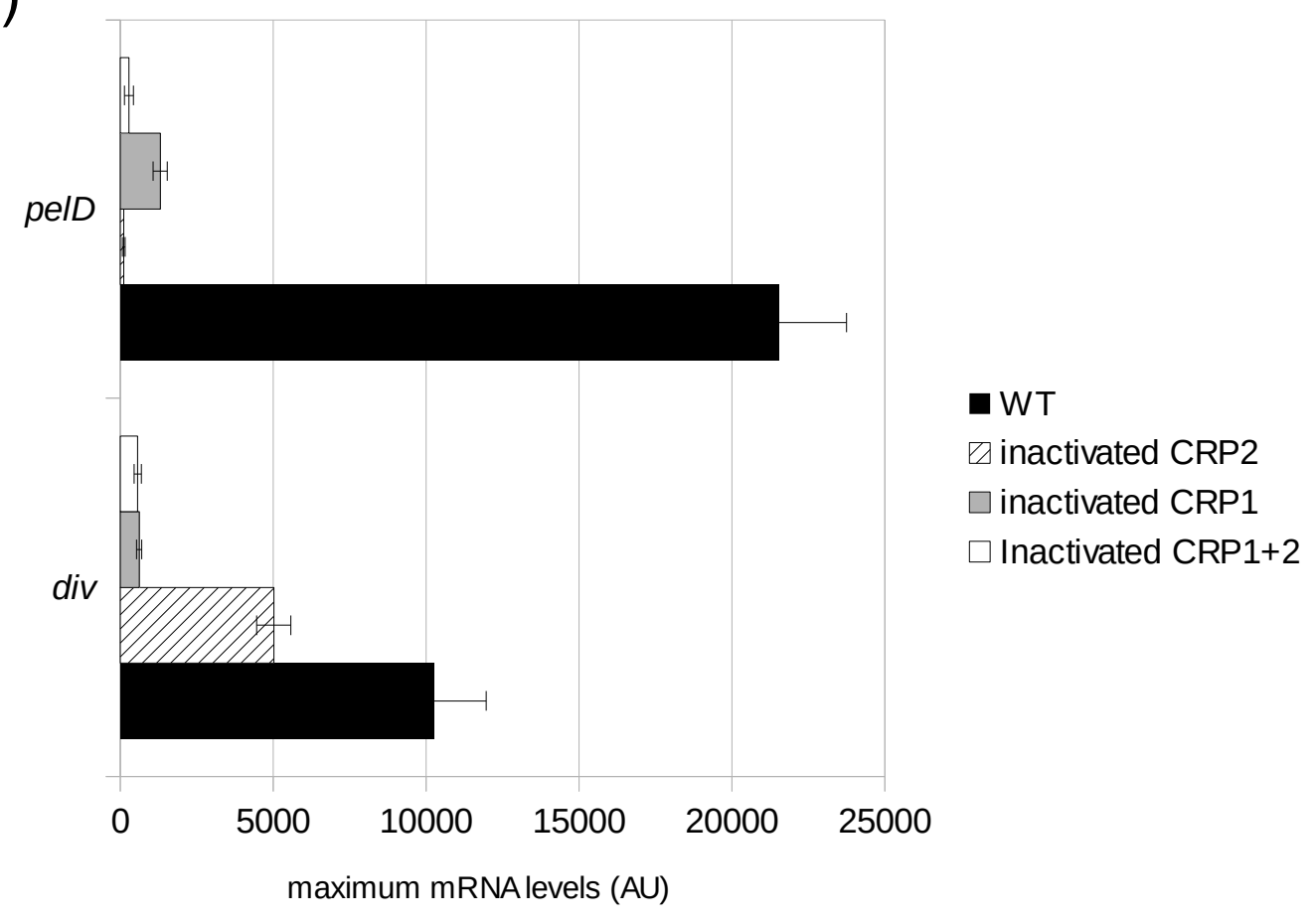


a)

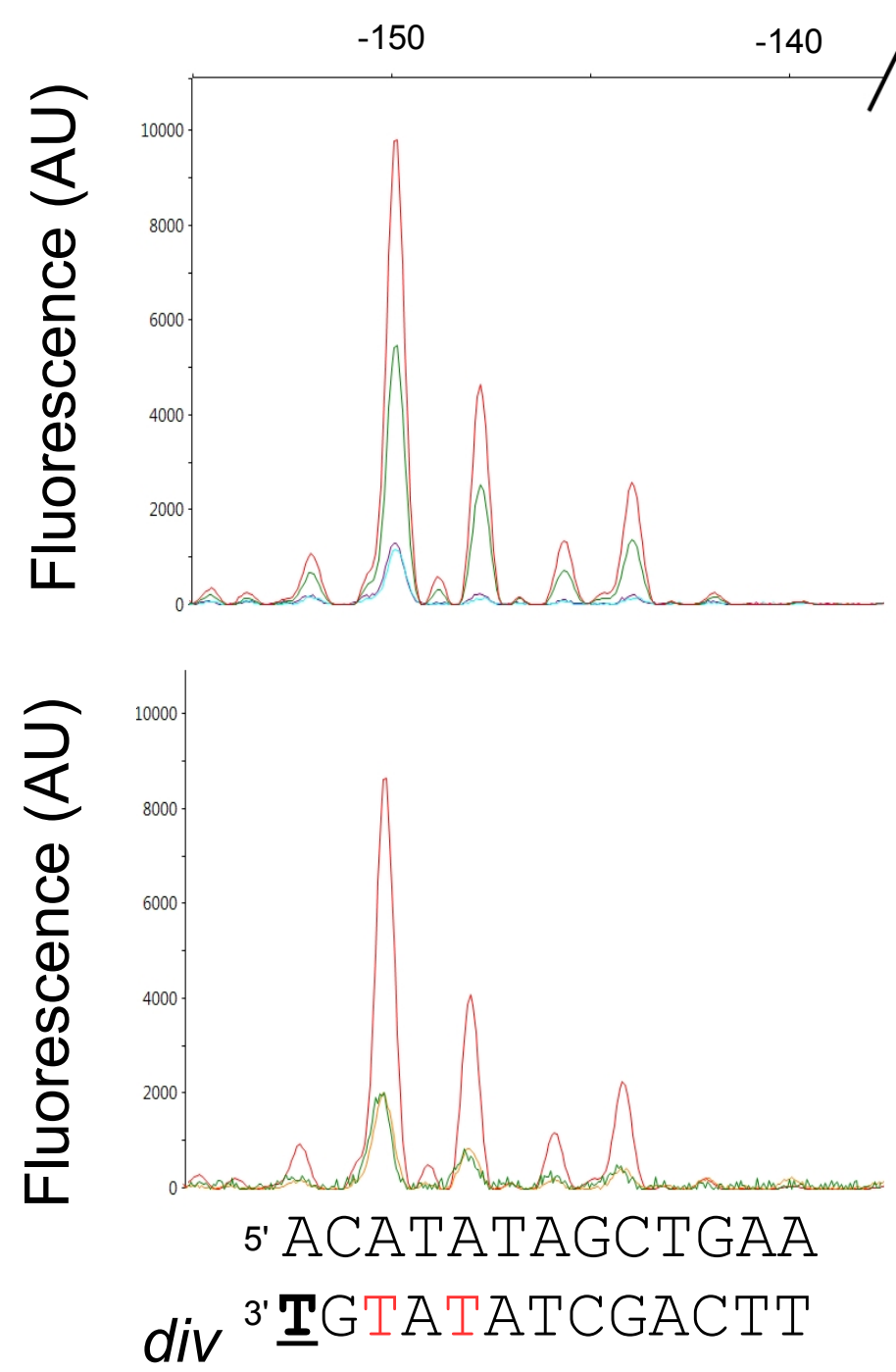

Position relatively to peID TSS

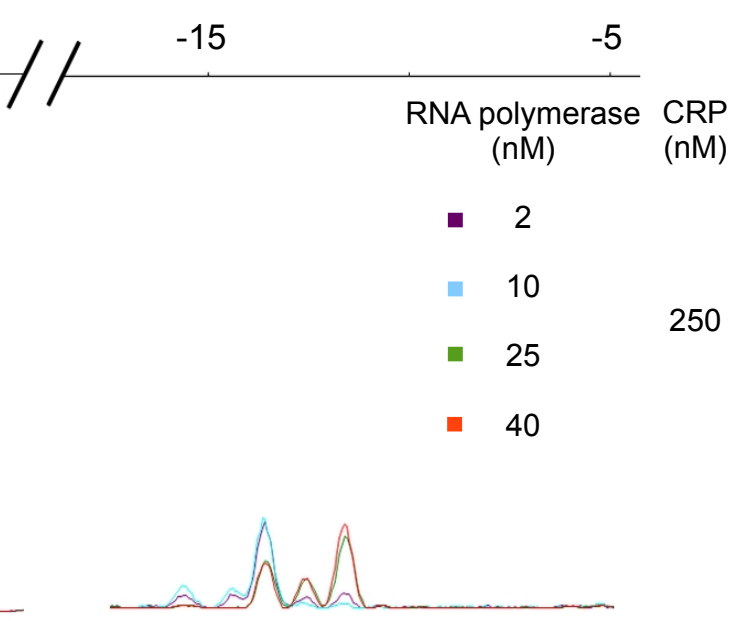

RNA polymerase

$$
\text { (nM) }
$$

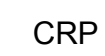

(nM)

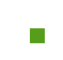

- 40

$-250$

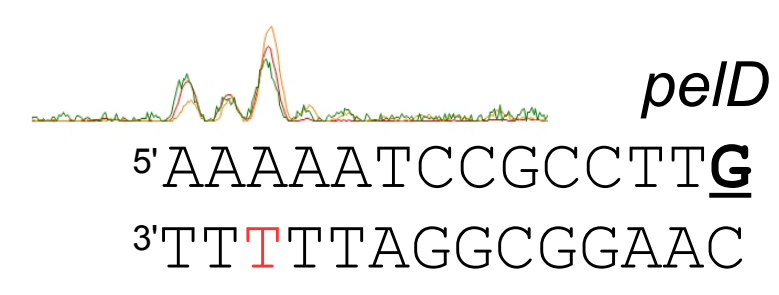

b)

pJD0 (WT)

pelD

RNA-I

div

[CRP] $0 \quad 100200400$

(nM) 


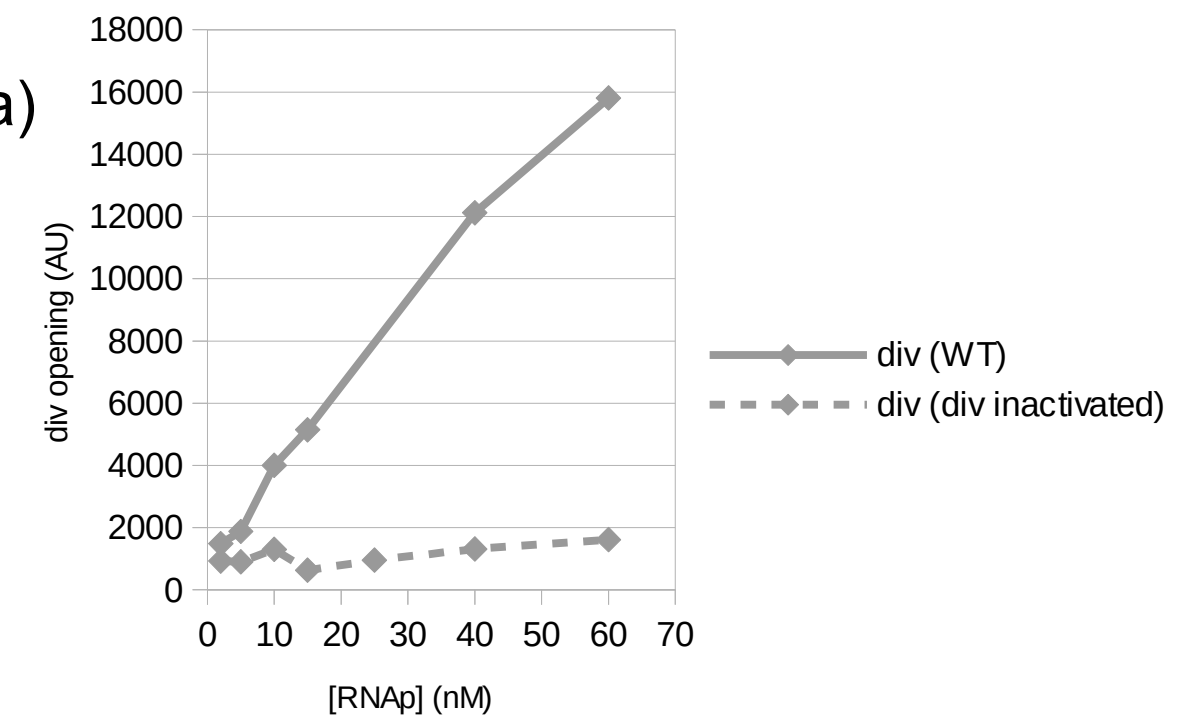

b)

fis
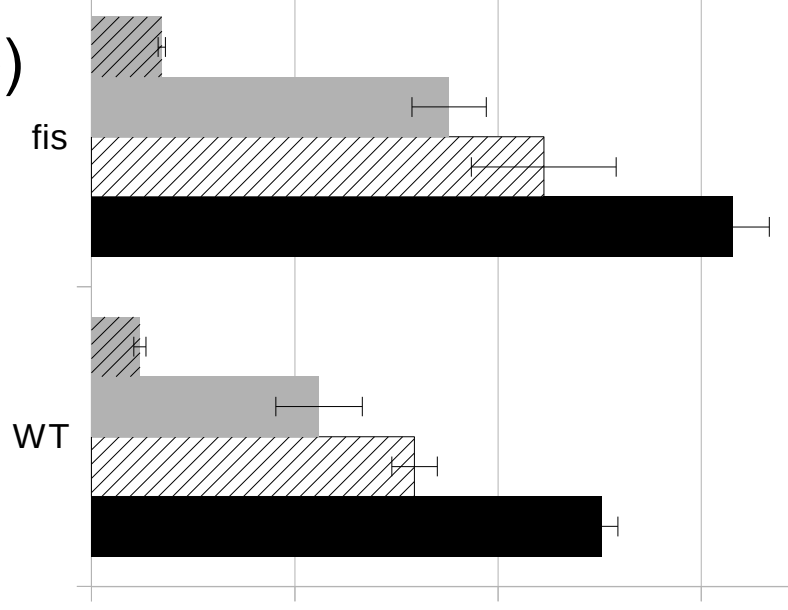

$10000 \quad 20000$

30000

Maximum mRNAlevels (AU) a pelD (WT)

$\square$ pelD (div inactivated) $\operatorname{div}(\mathrm{WT})$

$\checkmark$ div (div inactivated)
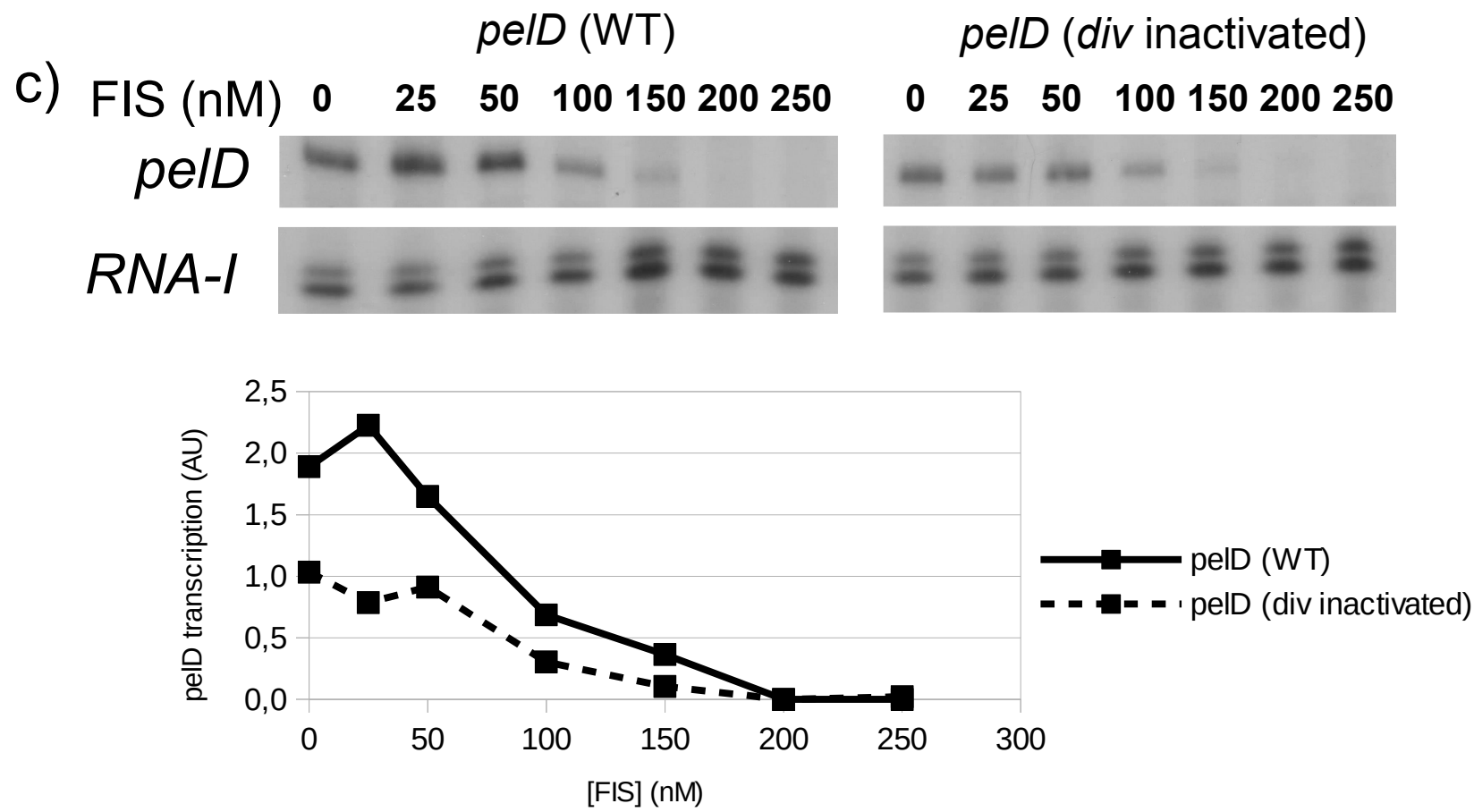


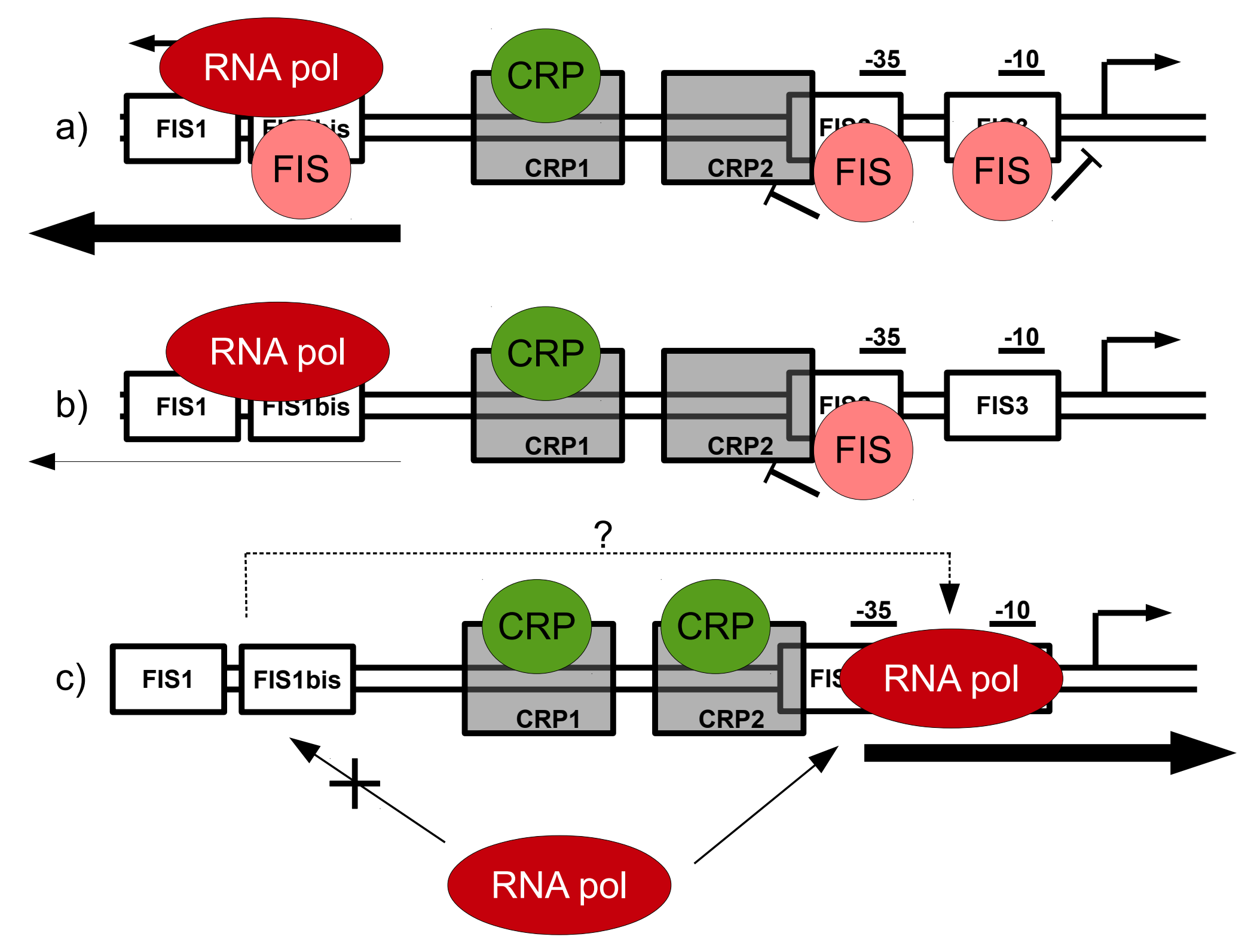




\section{Supplementary}


a)
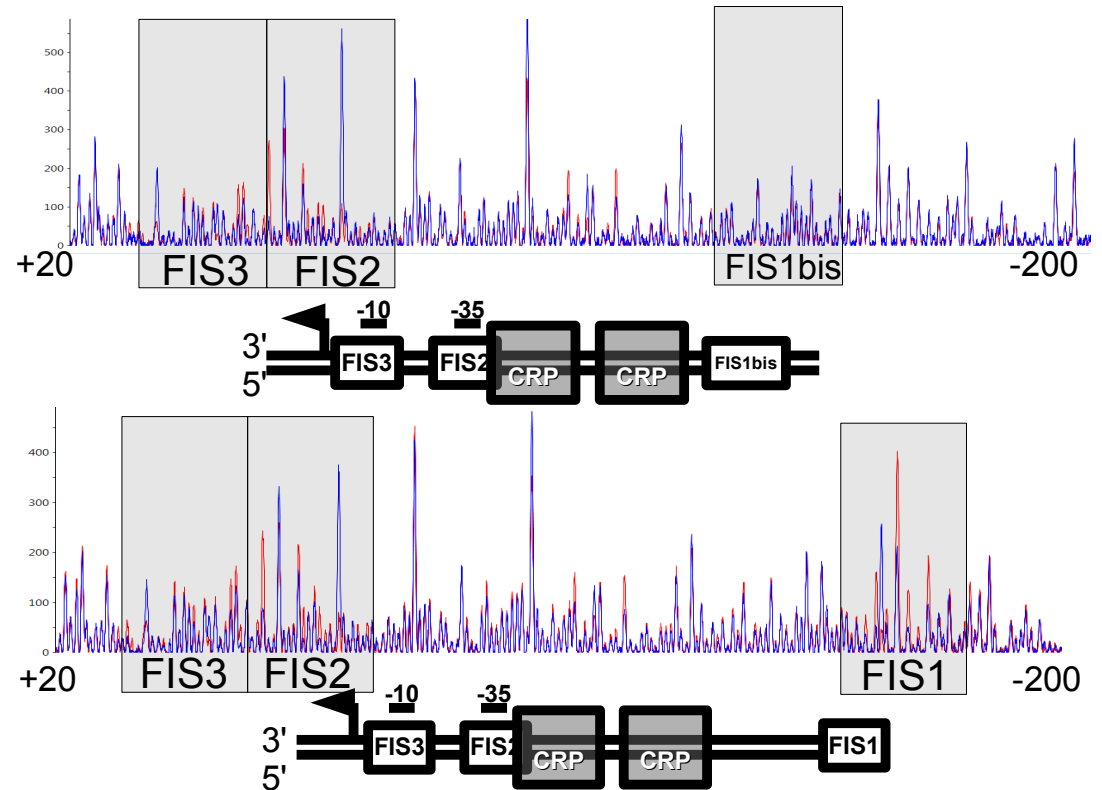

b)

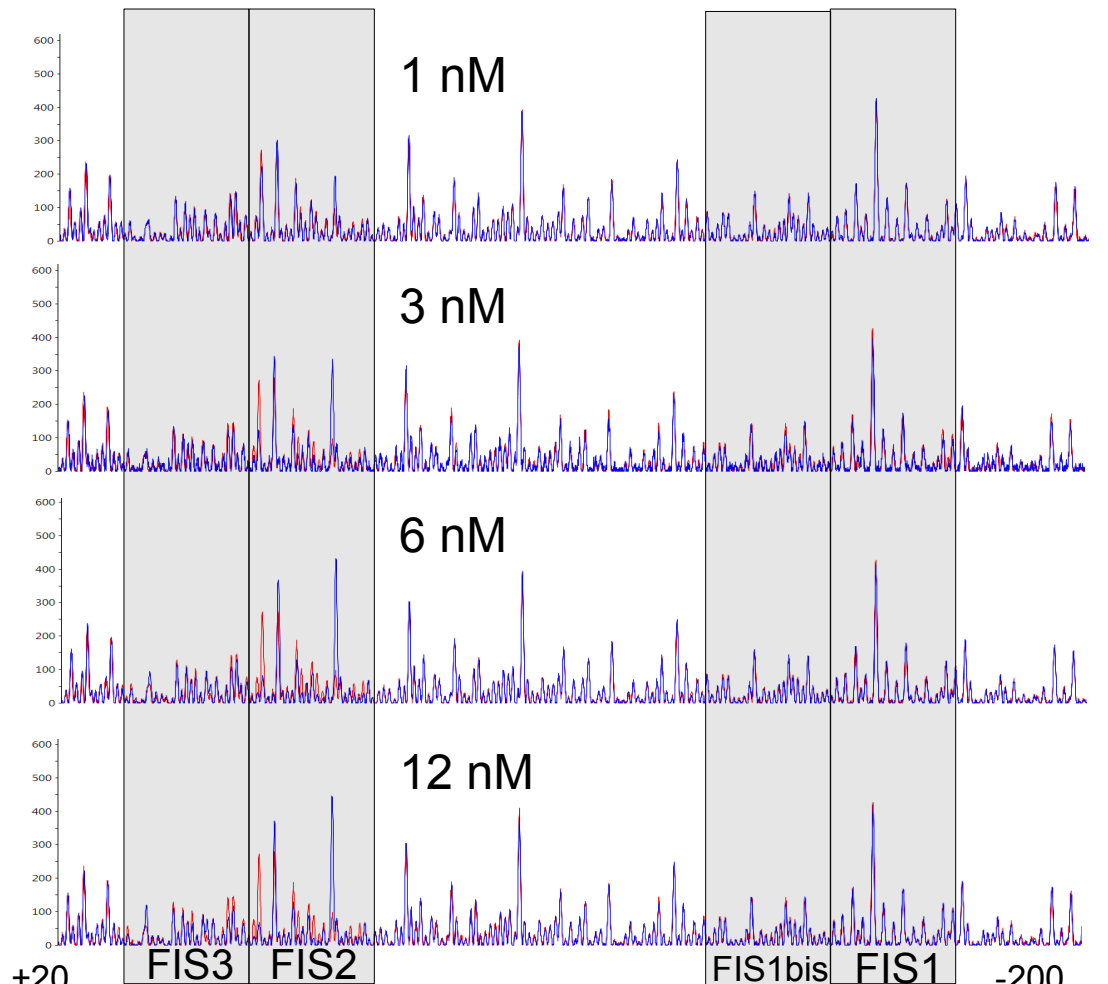

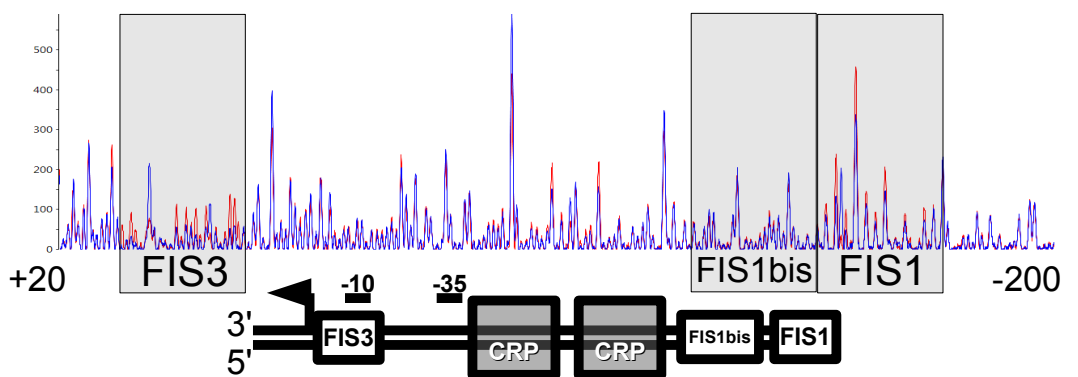
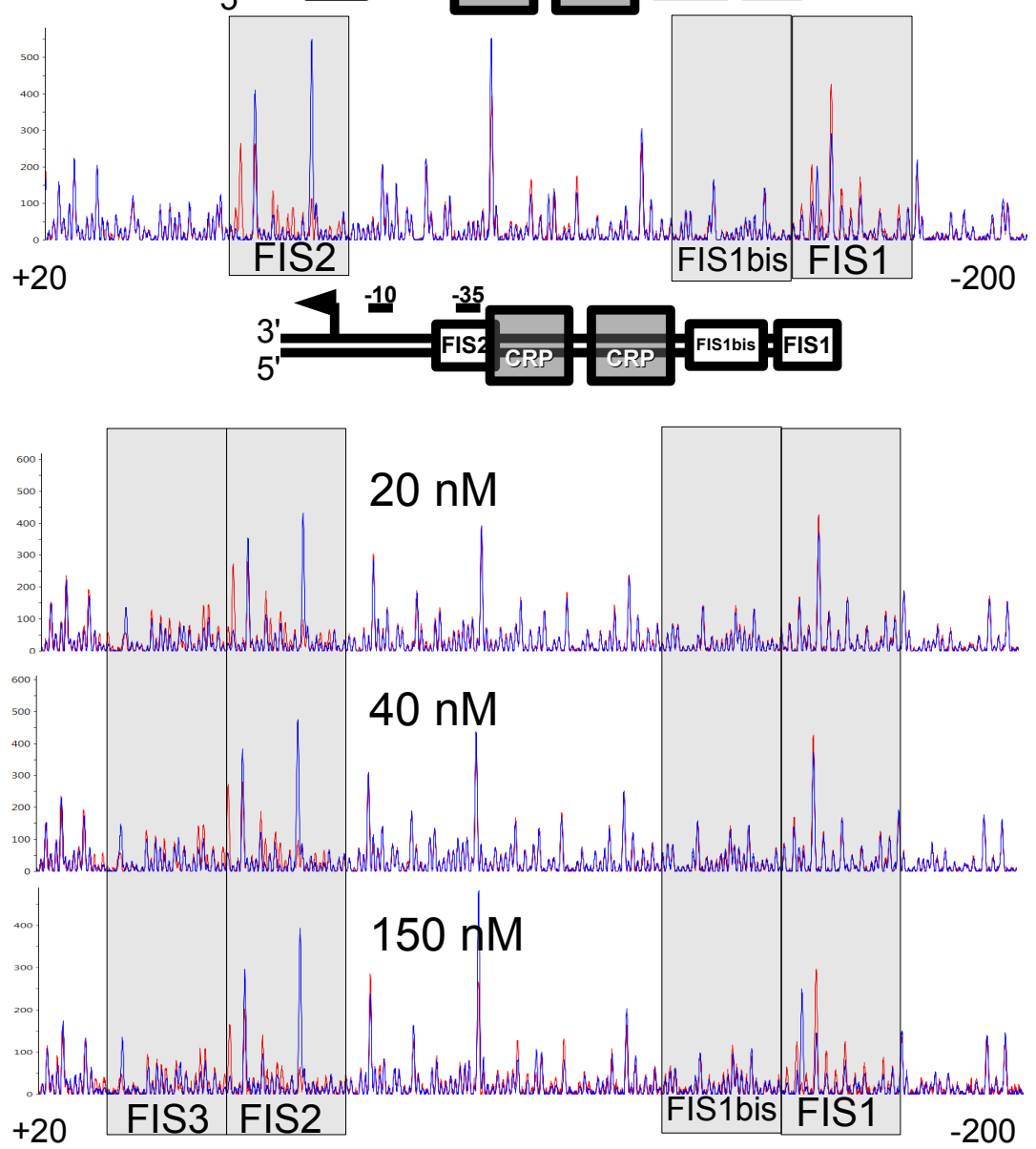

Fig S1: DNase I footprinting of FIS binding in the pelD regulatory region. DNase I footprinting was performed using fluorescently labelled linear peID DNA. The profiles represent the pattern obtained for the template strand. The red and the blue profiles correspond respectively to the samples without and with FIS. a) Profiles for peID DNA samples with inactivated FIS1, FIS1bis, FIS2 or FIS3 sites at 150 nM FIS concentration. b) Profiles of the wild type peID DNA incubated with increasing concentrations of FIS (from 1 to $150 \mathrm{nM}$ ) 


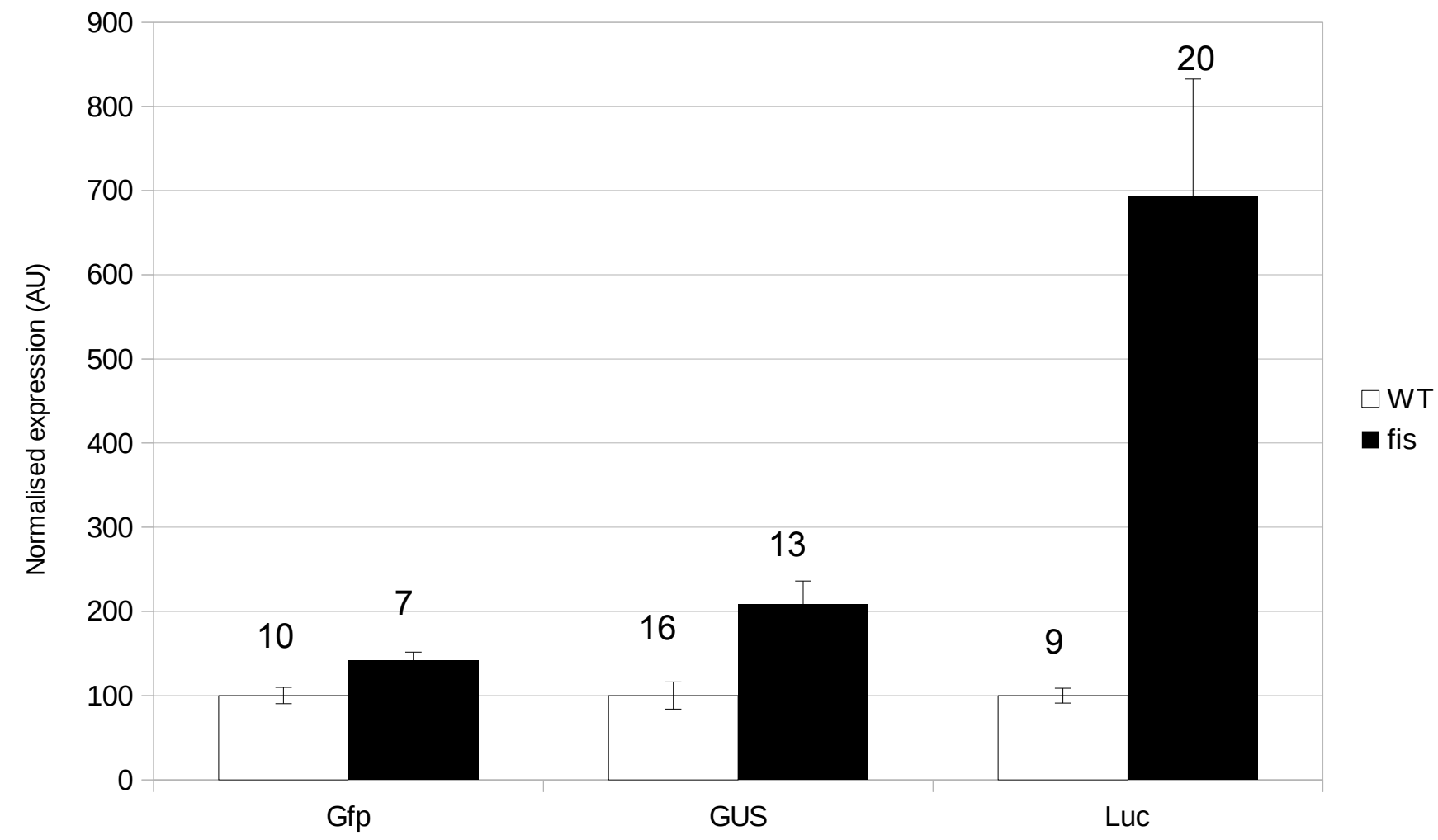

Fig S2: Comparison between the Gfp and Luc reporter systems in D. dadantii and the GUS system in E. coli. Bacteria transformed with the wild-type pelD regulatory region in a reporter plasmid were cultured in equivalent conditions (see materials and methods). The normalised fluorescence and GUS specific activity were taken in early stationary phase and the luminescence at the maximum. Data represents the mean of 3 (GUS) to 12 replicates (Gfp and Luc) and error bars represent the standard deviation. Numbers above the error bars indicate the coefficient of variation (in \%). 

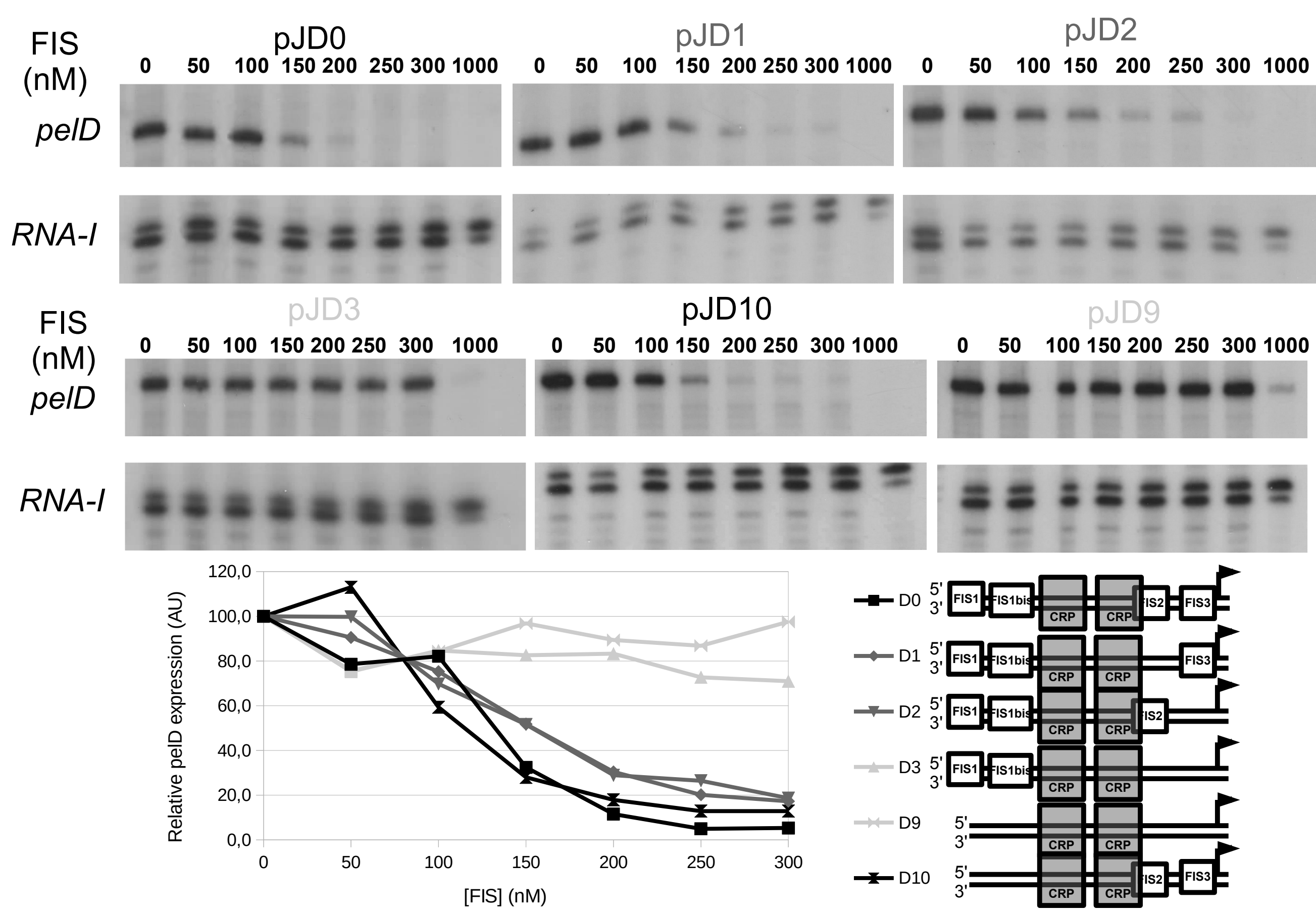

Fig S3: Effect of FIS binding on peID transcription in vitro. In vitro transcription was performed using several pelD constructs (plasmids D0, D1, D2, D3, D9 and D10 indicated in the bottom left panel) in presence of $100 \mathrm{nM}$ CRP, 40 nM RNA polymerase and varying concentrations of FIS. The RNA-I internal control was used as a standard for quantification of the pelD transcript (bottom right panel). 

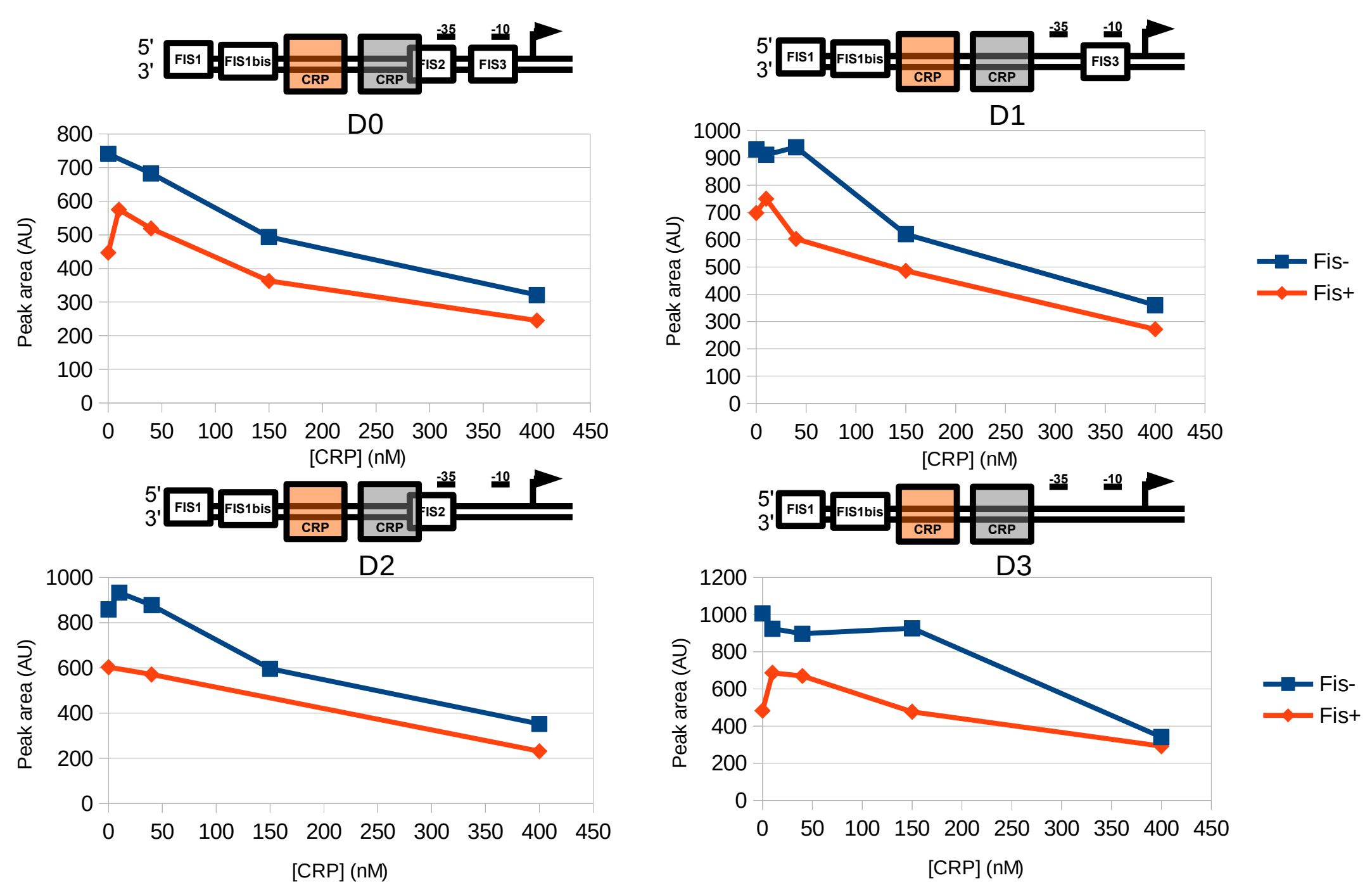

Fig S4: Absence of modification of the CRP1 DNase I footprint by the presence of FIS. A quantitative DNase I footprint was performed on fluorescently labelled peID regulatory regions, in identical conditions as in figure 5 . The area of the peaks at one position inside CRP1 (at position -85 relatively to the transcription start site) for different CRP concentrations is represented. 
a)

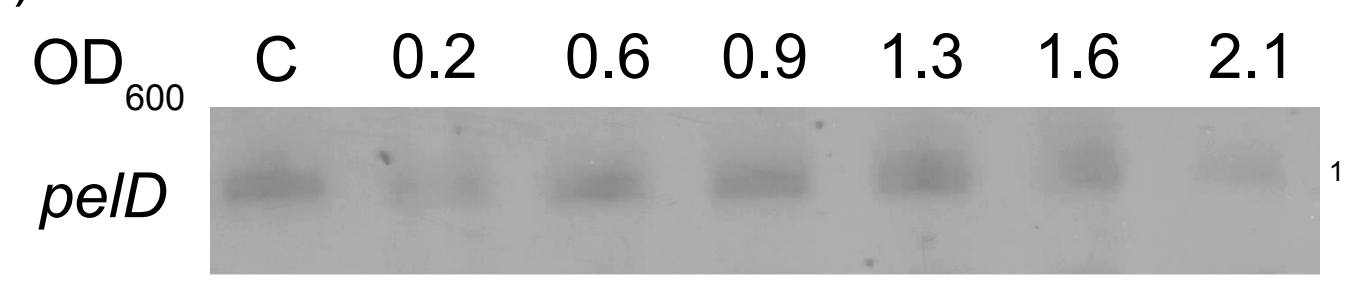

c)

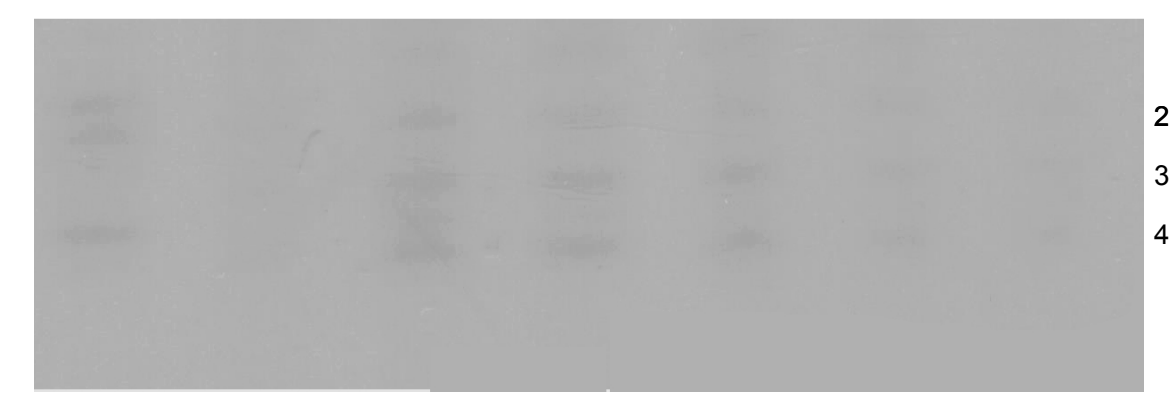

div

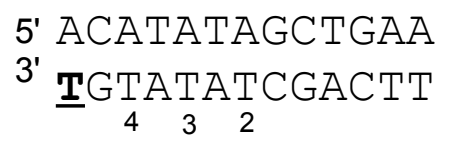

5' ACATATAGCTGAA $3-2$ b)

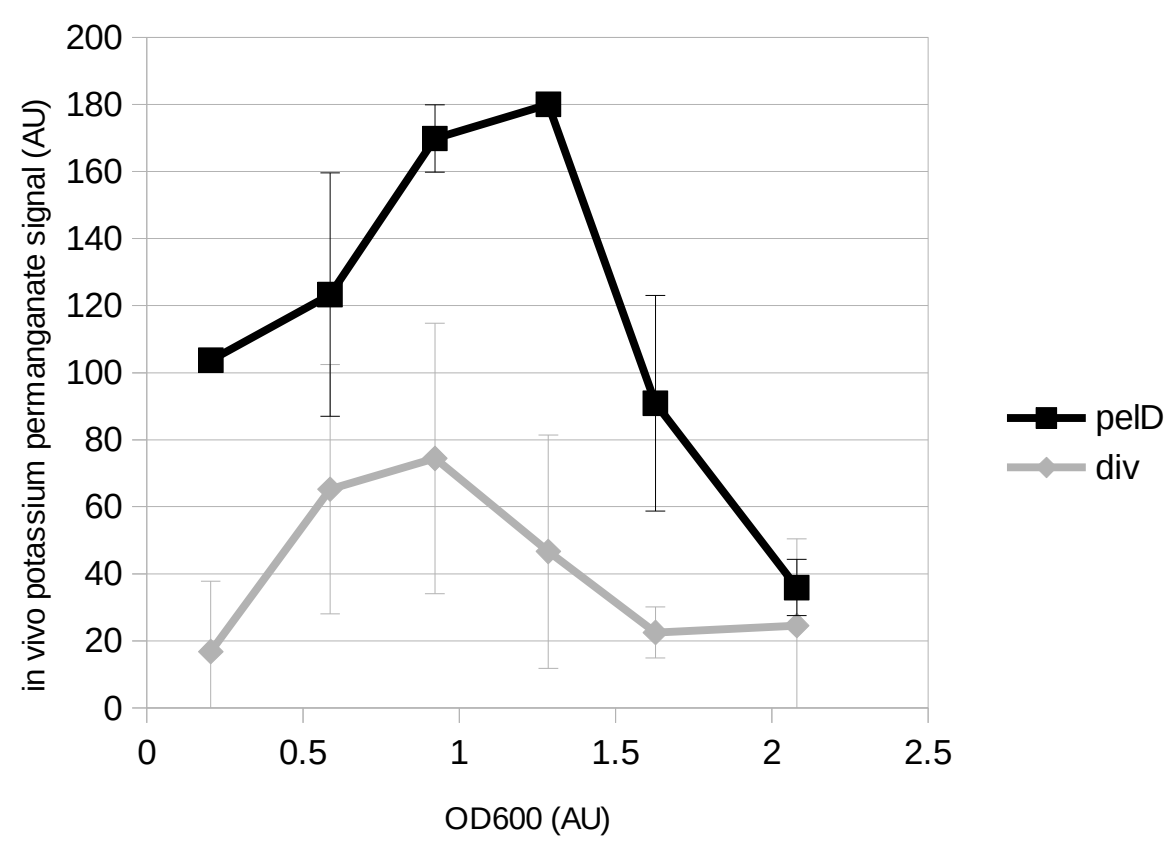

5' AAAAA TCCGCCTT $\underline{\mathbf{G}}$ 3' тTTTTAGGCGGAACG

Fig S5: in vivo potassium permanganate footprinting of the pelD regulatory region. pKD0 (wild-type pelD) was transformed into $D$. dadantii. Bacteria were then cultured in M63S + PGA and an in vivo potassium permanganate footprinting was performed as described in materials and methods. (a) image of one replicate of the experiment. Lane $\mathrm{C}$ corresponds to an in vitro potassium permanganate footprinting control performed on the same plasmid with $100 \mathrm{nM}$ CRP and $40 \mathrm{nM}$ RNAP. Bands are numbered and localised in part c of the figure. (b) quantification of the intensity of the bands, represented as mean +/- SD of 2 independent biological replicates. (c) Position of the attacked nucleotides of part a. Both strands are shown and transcription start sites are underlined. 
a)

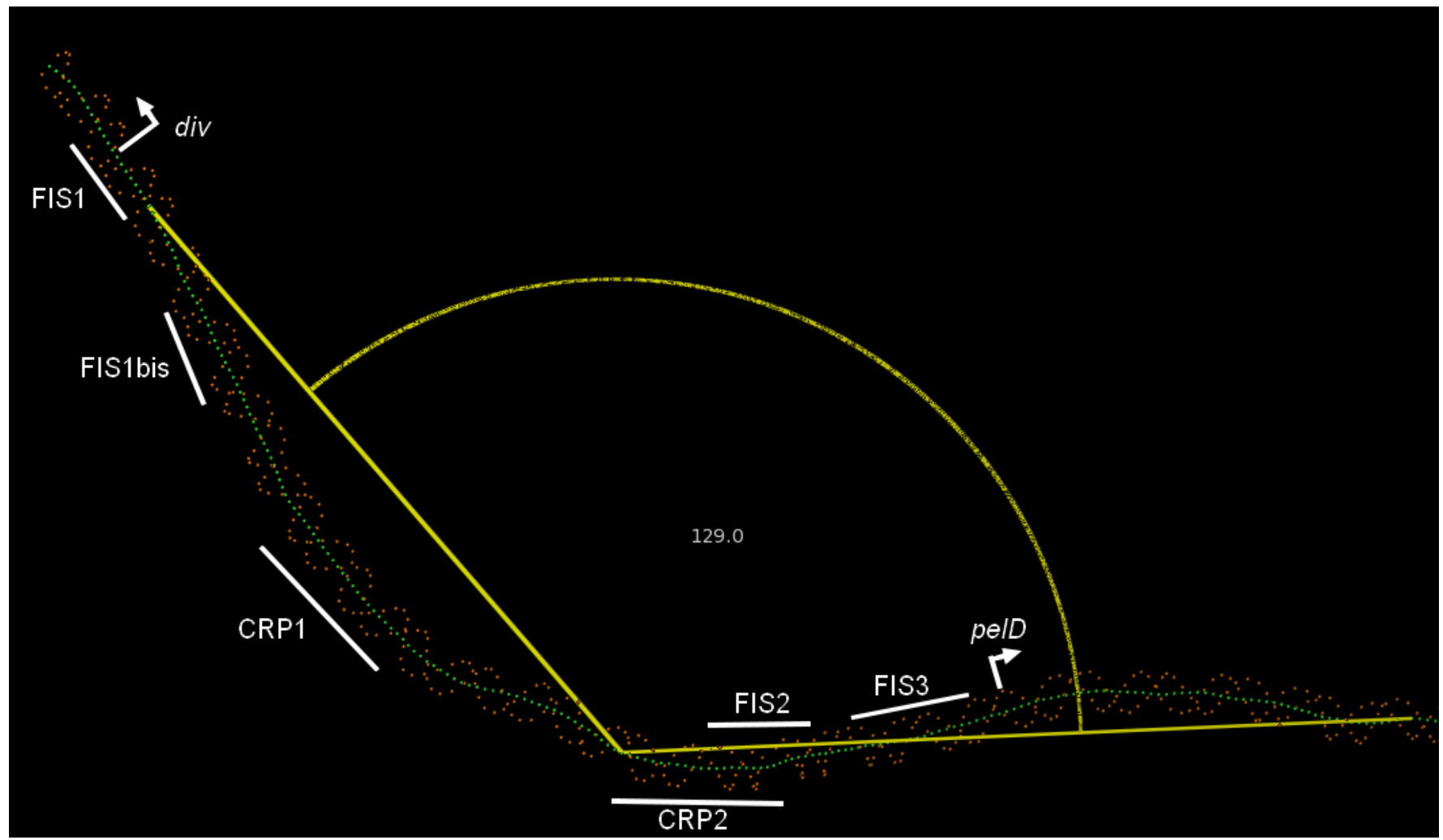

b)

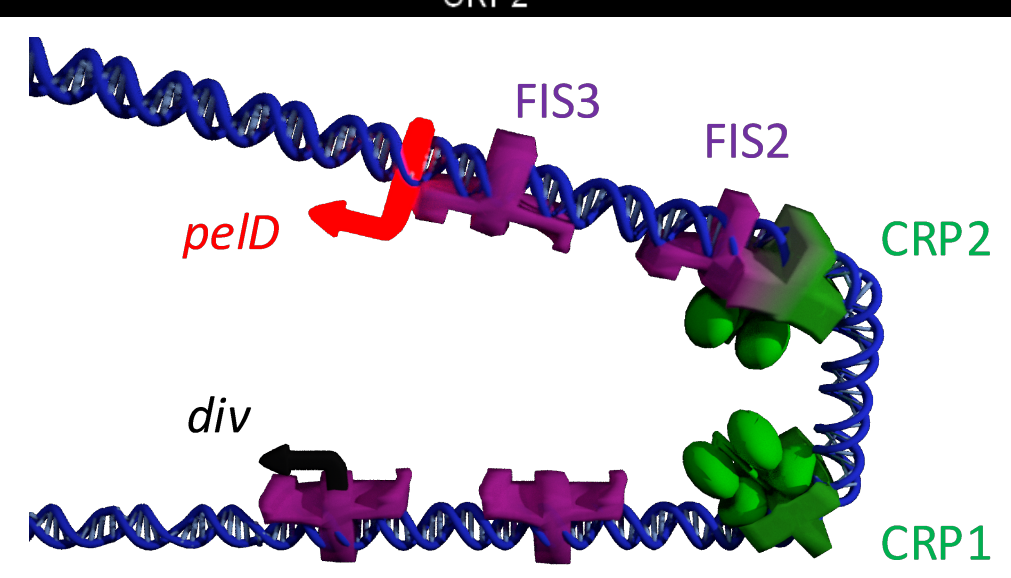

\section{FIS1 FIS1bis}

Fig S6: Predicted intrinsic and CRP-induced bending in the peID regulatory region. a) The intrinsic curvature of the pelD was modeled using DNAcurve (http://www.Ifd.uci.edu/ gohlke/dnacurve/) and the angle (in yellow) was measured using PyMOL, which corresponds to a bending angle of $51^{\circ}$. The DNA molecule is represented by orange dots and its trajectory in green dots. The main features of the pelD regulatory region are shown in white. b) Schematic representation of the CRP-induced bend in the pelD regulatory region. Considering that CRP can bend DNA to an angle of around $90^{\circ}$, the presence of both CRP sites could bring peID and div in close proximity, facilitating RNAP transfer between both. 


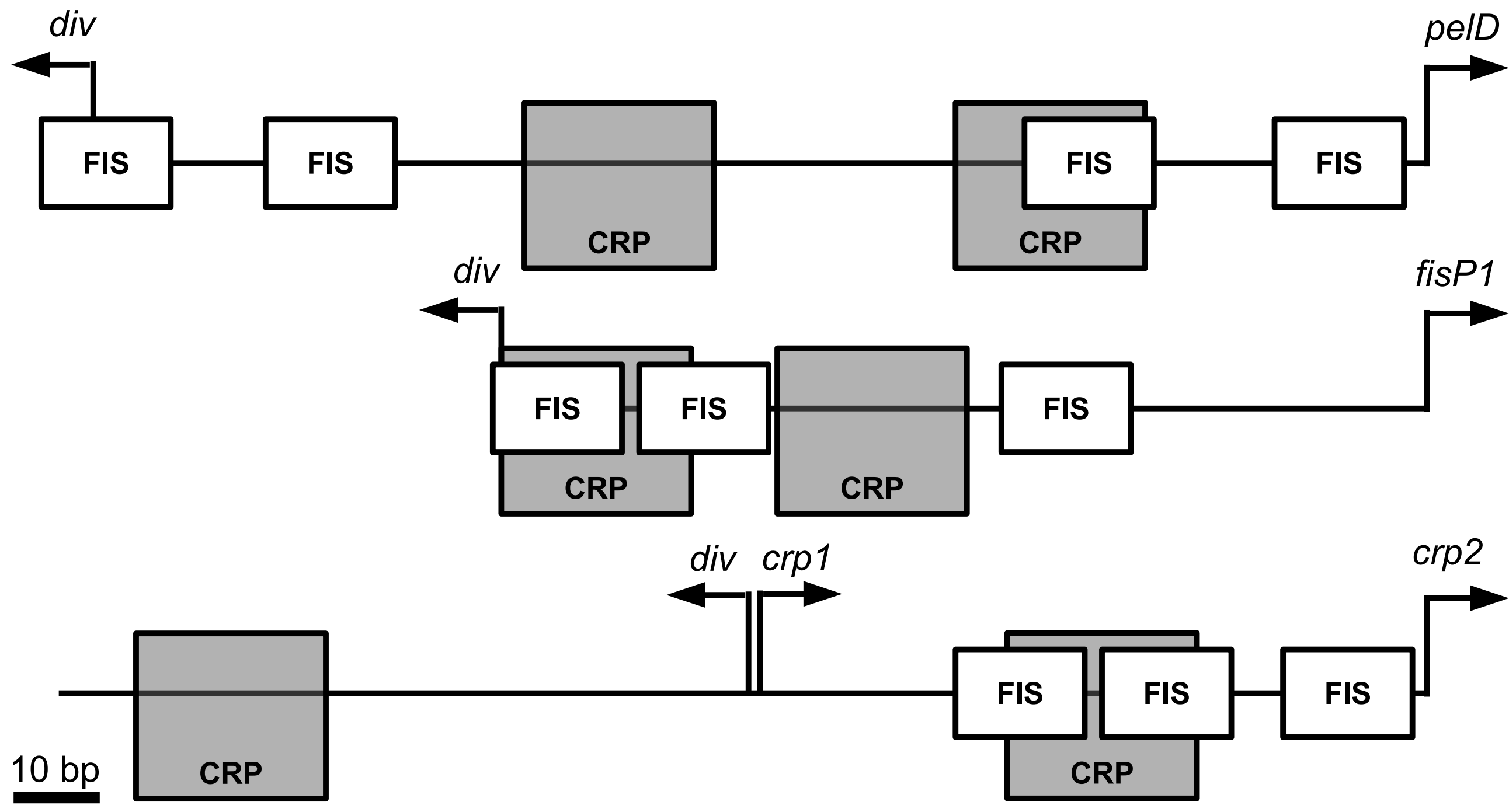

Fig S7: Regulatory structure of 3 different relevant promoters regulated by a divergent promoter. The pelD $(D$. dadantii), fis $(E$. coli) and $\operatorname{crp}(E$. coli) regulatory regions are represented. The known promoters are represented with an arrow, FIS sites by white boxes and CRP sites by gray boxes, as described in the litterature and this work. The promoter which is coupled to div is drawn on the right. All regulatory regions and binding sites are represented to scale. The scale bar in the bottom left corner represents 10 base pairs. 


\section{Supplementary table S1: strains, plasmids and oligos used in this study}

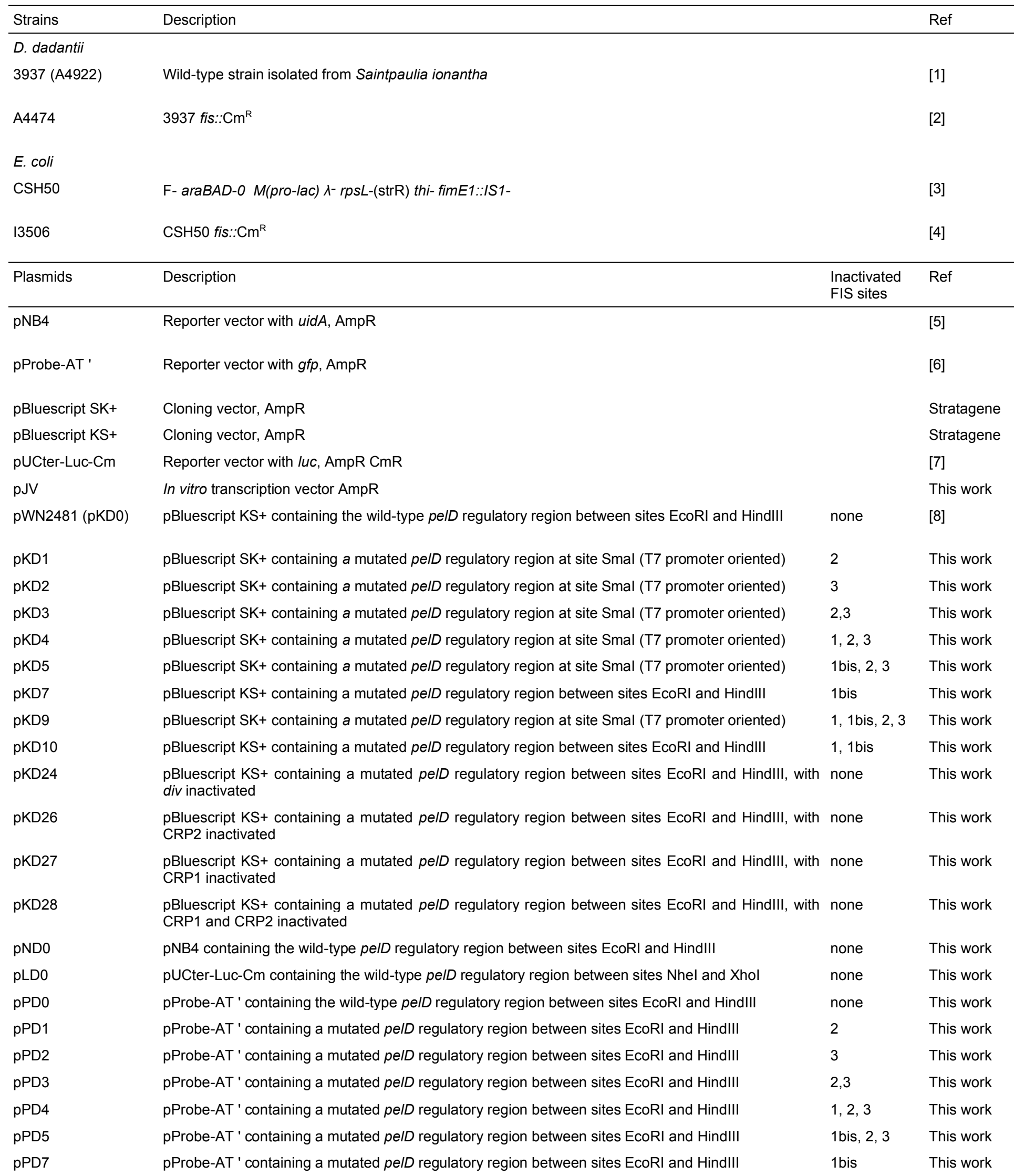




\begin{tabular}{|c|c|c|c|}
\hline pPD9 & pProbe-AT ' containing a mutated pelD regulatory region between sites EcoRI and HindIII & $1,1 \mathrm{bis}, 2,3$ & This work \\
\hline pPD24 & $\begin{array}{l}\text { pProbe-AT ' containing a mutated peID regulatory region between sites EcoRI and Hindlli, with div } \\
\text { inactivated }\end{array}$ & none & This work \\
\hline pPD26 & $\begin{array}{l}\text { pProbe-AT ' containing a mutated peID regulatory region between sites EcoRI and HindIII, with CRP2 } \\
\text { inactivated }\end{array}$ & none & This work \\
\hline pPD27 & $\begin{array}{l}\text { pProbe-AT ' containing a mutated peID regulatory region between sites EcoRI and HindIII, with CRP1 } \\
\text { inactivated }\end{array}$ & none & This work \\
\hline pPD28 & $\begin{array}{l}\text { pProbe-AT ' containing a mutated peID regulatory region between sites EcoRI and HindIII, with CRP1 } \\
\text { and CRP2 inactivated }\end{array}$ & none & This work \\
\hline pJD0 & pJV containing the wild-type pelD regulatory region between sites EcoRI and Sall & none & This work \\
\hline pJD1 & pJV containing a mutated pelD regulatory region between sites EcoRI and Sall & 2 & This work \\
\hline pJD2 & pJV containing a mutated peID regulatory region between sites EcoRI and Sall & 3 & This work \\
\hline pJD3 & pJV containing a mutated peID regulatory region between sites EcoRI and Sall & 2,3 & This work \\
\hline pJD9 & pJV containing a mutated peID regulatory region between sites EcoRI and Sall & $1,1 \mathrm{bis}, 2,3$ & This work \\
\hline pJD10 & pJV containing a mutated peID regulatory region between sites EcoRI and Sall & $1,1 \mathrm{bis}$ & This work \\
\hline pJD24 & pJV containing a mutated pelD regulatory region between sites EcoRI and Sall, with div inactivated & none & This work \\
\hline pID0 & pProbe-AT ' containing a wild-type peID regulatory region between sites $\mathrm{Kpnl}$ and $\mathrm{BamHI}$ & none & This work \\
\hline pID24 & $\begin{array}{l}\text { pProbe-AT ' containing a mutated pelD regulatory region between sites } \mathrm{Kpnl} \text { and } \mathrm{BamHI} \text {, with div } \\
\text { inactivated }\end{array}$ & none & This work \\
\hline pID26 & $\begin{array}{l}\text { pProbe-AT ' containing a mutated pelD regulatory region between sites } \mathrm{Kpnl} \text { and } \mathrm{BamHI} \text {, with CRP2 } \\
\text { inactivated }\end{array}$ & none & This work \\
\hline pID27 & $\begin{array}{l}\text { pProbe-AT ' containing a mutated pelD regulatory region between sites } \mathrm{Kpnl} \text { and BamHI, with CRP1 } \\
\text { inactivated }\end{array}$ & none & This work \\
\hline pID28 & $\begin{array}{l}\text { pProbe-AT ' containing a mutated pelD regulatory region between sites } \mathrm{Kpnl} \text { and } \mathrm{BamHI} \text {, with CRP1 } \\
\text { and CRP2 inactivated }\end{array}$ & none & This work \\
\hline Primers & & & Ref \\
\hline DNase pel FW & HEX-ACTATAGGGCGAATTGG & & This work \\
\hline DNase pel REV & FAM-AATTAACCCTCACTAAAGG & & This work \\
\hline B0014 FW & AACAGCTGTCACACTGGCTCACCTTC & & This work \\
\hline B0014 REV & AAGAATTCAATAATAAAAAAGCCGGATTAATAATC & & This work \\
\hline bla 3B4 fluo & HEX-CAGGAAGGCAAAATGCCGC & & This work \\
\hline pelDfis1bismut FW & GCTGAATTTAAAAtAAAAATTAATTCAAtATTCATAACTAAAAG & & This work \\
\hline pelDfis1bismut REV & CTTTTAGTTATGAATaTTGAATTAATTTTTTaTTTTAAATTCAGC & & This work \\
\hline pelDFis1mutFW & TTTCAGATAAAAACtCTTATACATATAGtTGAATTTAAAA & & This work \\
\hline pelDFis1mutREV & TTTTAAATTCAaCTATATGTATAAGaGTTTTTATCTGAAA & & This work \\
\hline 35rev v2 FW & GAATTTAAAAGAAAAATTAATTCttCATTCATAACTAAAAGTTACC & & This work \\
\hline 35rev v2 REV & GGTAACTTTTAGTTATGAATGaaGAATTAATTTTTTCTTTTAAATTC & & This work \\
\hline 300 & ACTATAGGGCGAATTGG & & This work \\
\hline 395 & CATAAAAAAAACGAGATTTTGAagtCAAAATAAACAATCGAAAACGC & & This work \\
\hline 396 & GCGTTTTCGATTGTTTATTTTGactTCAAAATCTCGTTTTTTTTATG & & This work \\
\hline 397 & CTAAAAGTTACCGGTCACGAagtCACTTTAGATAAAATTAATTAGC & & This work \\
\hline 398 & GCTAATTAATTTTATCTAAAGTGactTCGTGACCGGTAACTTTTAG & & This work \\
\hline
\end{tabular}

\section{References}

[1] A. Kotoujansky, M. Lemattre, P. Boistard, Utilization of a thermosensitive episome bearing transposon TN10 to isolate Hfr donor strains of 
Erwinia carotovora subsp. chrysanthemi., J. Bacteriol. 150 (1982) 122-131.

[2] T. Lautier, W. Nasser, The DNA nucleoid-associated protein Fis co-ordinates the expression of the main virulence genes in the phytopathogenic bacterium Erwinia chrysanthemi, Mol. Microbiol. 66 (2007) 1474-1490. doi:10.1111/j.1365-2958.2007.06012.x.

[3] Miller JH, Experiment in Molecular Genetics, Cold Spring Harbor Laboratory Press, 1972.

[4] G. González-Gil, P. Bringmann, R. Kahmann, FIS is a regulator of metabolism in Escherichia coli, Mol. Microbiol. 22 (1996) $21-29$. doi:10.1111/j.1365-2958.1996.tb02652.x

[5] N. Bardonnet, A. Trautwetter, G. Couchoux-Luthaud, C. Blanco, Plasmids with the uidA reporter gene for the detection of promoters and transcription signals, Mol. Gen. Genet. MGG. 212 (1988) 390-392.

[6] W.G. Miller, J.H. Leveau, S.E. Lindow, Improved gfp and inaZ broad-host-range promoter-probe vectors, Mol. Plant-Microbe Interact. MPMI. 13 (2000) 1243-1250. doi:10.1094/MPMI.2000.13.11.1243.

[7] X. Jiang, P. Sobetzko, W. Nasser, S. Reverchon, G. Muskhelishvili, Chromosomal "Stress-Response" Domains Govern the Spatiotemporal Expression of the Bacterial Virulence Program, mBio. 6 (2015) e00353-15. doi:10.1128/mBio.00353-15.

[8] C. Rouanet, K. Nomura, S. Tsuyumu, W. Nasser, Regulation of pelD and pelE, Encoding Major Alkaline Pectate Lyases in Erwinia chrysanthemi: Involvement of the Main Transcriptional Factors, J. Bacteriol. 181 (1999) 5948-5957. 